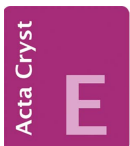

CRYSTALLOGRAPHIC COMMUNICATIONS

ISSN 2056-9890

\title{
Crystal structures and hydrogen-bonding analysis of a series of solvated ammonium salts of molybdenum(II) chloride clusters
}

\author{
Dean H. Johnston* and Ikponmwosa Agho
}

Department of Chemistry, Otterbein University, Westerville, $\mathrm{OH} 43081$, USA. *Correspondence e-mail: djohnston@otterbein.edu

Received 18 July 2019

Accepted 9 October 2019

Edited by E. V. Boldyreva, Russian Academy of Sciences, Russia

Keywords: metal-halide cluster; charge-assisted hydrogen bonds; primary ammonium salt; quaternary ammonium salt; Hirshfeld surface; crystal structure.

CCDC references: 1907244; 1907230; 1907249; 1907231

Supporting information: this article has supporting information at journals.iucr.org/e

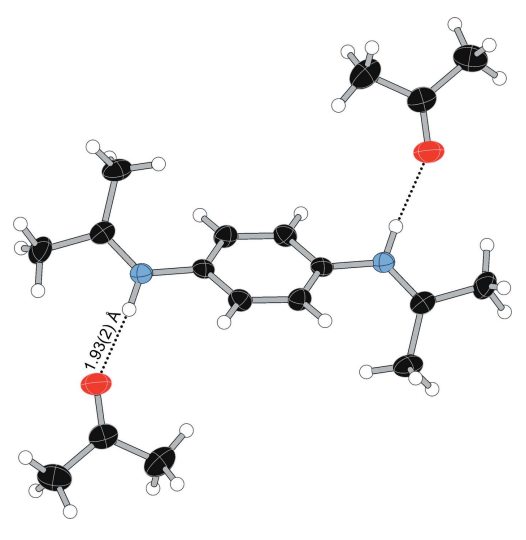

OPEN $\odot$ ACCESS
Charge-assisted hydrogen bonding plays a significant role in the crystal structures of solvates of ionic compounds, especially when the cation or cations are primary ammonium salts. We report the crystal structures of four ammonium salts of molybdenum halide cluster solvates where we observe significant hydrogen bonding between the solvent molecules and cations. The crystal structures of bis(anilinium) octa- $\mu_{3}$-chlorido-hexachlorido-octahedro-hexamolybdate $N, N$-dimethylformamide tetrasolvate, $\left(\mathrm{C}_{6} \mathrm{H}_{8} \mathrm{~N}\right)_{2}\left[\mathrm{Mo}_{6} \mathrm{Cl}_{8} \mathrm{Cl}_{6}\right] \cdot-$ $4 \mathrm{C}_{3} \mathrm{H}_{7} \mathrm{NO}$, (I), p-phenylenediammonium octa- $\mu_{3}$-chlorido-hexachlorido-octahedro-hexamolybdate $N, N$-dimethylformamide hexasolvate, $\left(\mathrm{C}_{6} \mathrm{H}_{10} \mathrm{~N}_{2}\right)\left[\mathrm{Mo}_{6}\right.$ $\left.\mathrm{Cl}_{8} \mathrm{Cl}_{6}\right] \cdot 6 \mathrm{C}_{3} \mathrm{H}_{7} \mathrm{NO}, \quad(\mathbf{I I}), \quad N, N^{\prime}$-(1,4-phenylene)bis(propan-2-iminium) octa- $\mu_{3^{-}}$ chlorido-hexachlorido-octahedro-hexamolybdate acetone trisolvate, $\left(\mathrm{C}_{12} \mathrm{H}_{18} \mathrm{~N}_{2}\right)$ $\left[\mathrm{Mo}_{6} \mathrm{Cl}_{8} \mathrm{Cl}_{6}\right] \cdot 3 \mathrm{C}_{3} \mathrm{H}_{6} \mathrm{O}$, (III), and 1,1'-dimethyl-4,4'-bipyridinium octa- $\mu_{3}$-chlorido-hexachlorido-octahedro-hexamolybdate $\mathrm{N}, \mathrm{N}$-dimethylformamide tetrasolvate, $\left(\mathrm{C}_{12} \mathrm{H}_{14} \mathrm{~N}_{2}\right)\left[\mathrm{Mo}_{6} \mathrm{Cl}_{8} \mathrm{Cl}_{6}\right] \cdot 4 \mathrm{C}_{3} \mathrm{H}_{7} \mathrm{NO}$, (IV), are reported and described. In (I), the anilinium cations and $N, N$-dimethylformamide (DMF) solvent molecules form a cyclic $R_{4}^{2}(8)$ hydrogen-bonded motif centered on a crystallographic inversion center with an additional DMF molecule forming a $D(2)$ interaction. The $p$-phenylenediammonium cation in (II) forms three $D(2)$ interactions between the three $\mathrm{N}-\mathrm{H}$ bonds and three independent $N, N$ dimethylformamide molecules. The dication in (III) is a protonated Schiff base solvated by acetone molecules. Compound (IV) contains a methyl viologen dication with $\mathrm{N}, \mathrm{N}$-dimethylformamide molecules forming close contacts with both aromatic and methyl $\mathrm{H}$ atoms.

\section{Chemical context}

The unique photochemistry of the molybdenum and tungsten halide clusters $\left[M_{6} X_{8} Y_{6}\right]^{2-}(M=\mathrm{Mo}, \mathrm{W} ; X, Y=\mathrm{Cl}, \mathrm{Br}, \mathrm{I})$ has been known for over 30 years (Maverick et al., 1983) and researchers continue to explore the tunabilty of the redox potentials, crystal structures and photochemical properties of cluster-containing compounds via variation of the bridging and terminal ligands and the counter-ion (Mikhailov et al., 2016; Saito et al., 2017; Akagi et al., 2018). Metal clusters, such as molybdenum halides, consist of an inner $\left[\mathrm{Mo}_{6} X_{8}\right]^{4+}$ core surrounded by six axial ligands which are more labile than the core ligands, making the preparation of mixed-ligand cluster complexes relatively straightforward.

Charge-assisted hydrogen bonds (CAHBs) are particularly strong among hydrogen bonds (Gilli \& Gilli, 2009) and can be a significant factor in the design and formation of supramolecular complexes. CAHBs have been exploited in the formation of supramolecular organic-inorganic uranyl materials (de Groot et al., 2014), noncovalent macrocycles and catenanes (Pop et al., 2016), molecular switches (Gurbanov et 


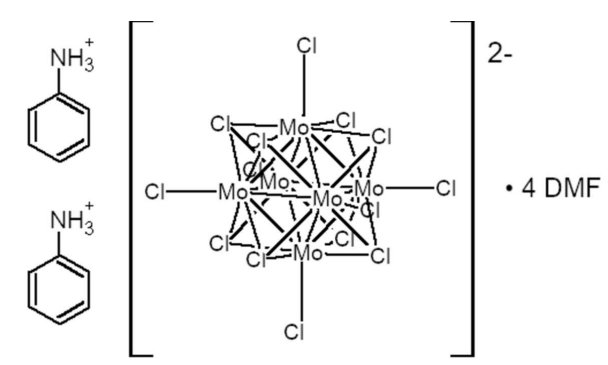

(1)

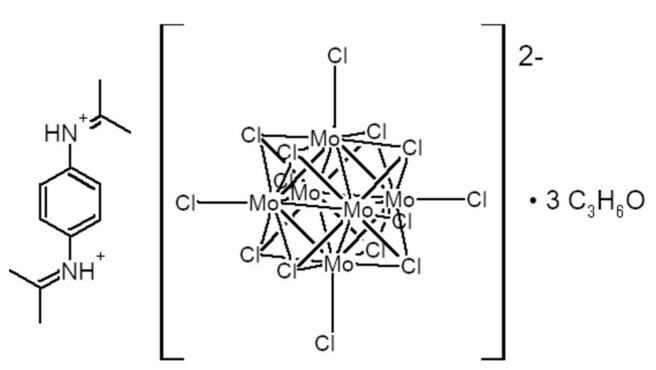

(3)

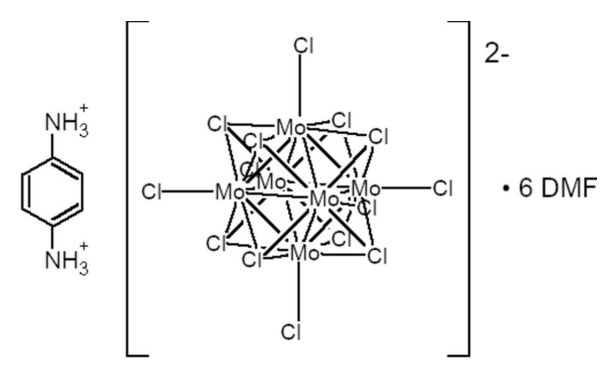

(2)

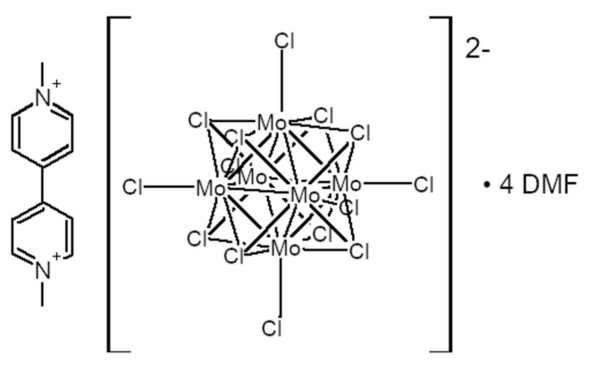

(4)

Figure 1

The structures of (I)-(IV).

al., 2017), and CAHB networks (Ward, 2009). Protonated diamines are a common motif found in hydrogen-bonded materials (Brozdowska \& Chojnacki, 2017; Zick \& Geiger, 2018). Examination of the nature and range of hydrogen bonding for solvates can provide information about the stability and physical properties of molecular solids (Brychczynska et al., 2012).

We have prepared a series of ammonium salts of the $\left[\mathrm{Mo}_{6} \mathrm{Cl}_{8} \mathrm{Cl}_{6}\right]^{2-}$ complex anion, each containing cations

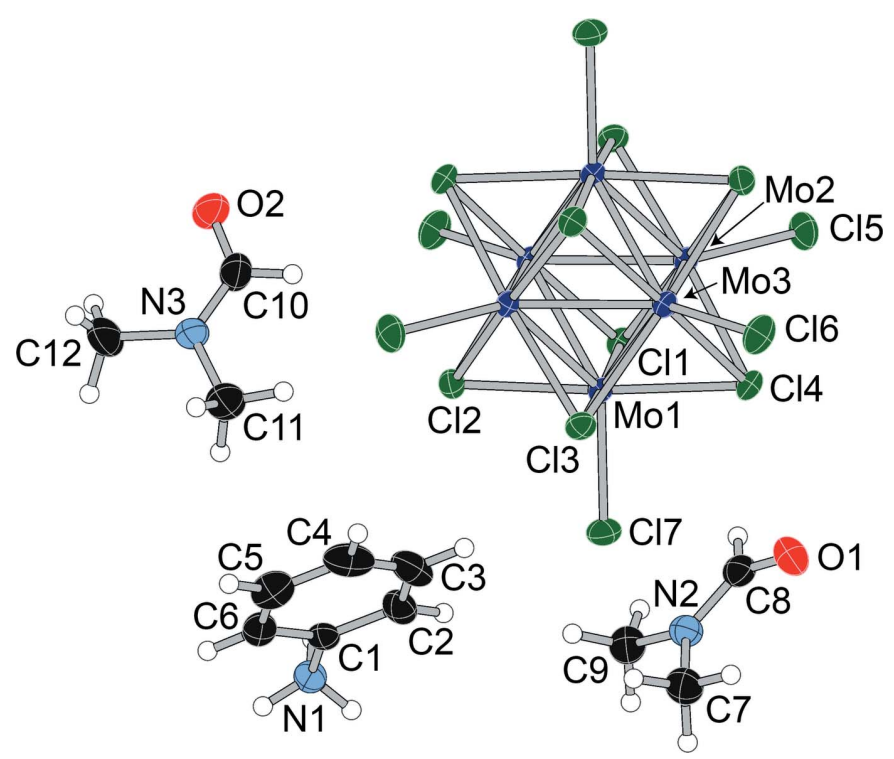

Figure 2

Displacement ellipsoid plot and atom-numbering scheme for (I), with ellipsoids drawn at the $50 \%$ probability level. 'solvated' by either dimethylformamide or acetone through strong CAHBs.

\section{Structural commentary}

The asymmetric unit of dianilinium salt (I) (Fig. 1) contains half a cluster unit, one anilinium cation, and two independent $\mathrm{N}, \mathrm{N}$-dimethylformamide (DMF) molecules. The structure with the atom-numbering scheme is shown in Fig. 2. The $\left[\mathrm{Mo}_{6} \mathrm{Cl}_{8} \mathrm{Cl}_{6}\right]^{2-}$ cluster unit resides on a crystallographic inversion center, as it does in all four structures. In compound (II), the asymmetric unit contains half a cluster unit, half a $p$-phenylenediammonium cation, and three independent DMF molecules. The $p$-phenylenediammonium cation is disordered

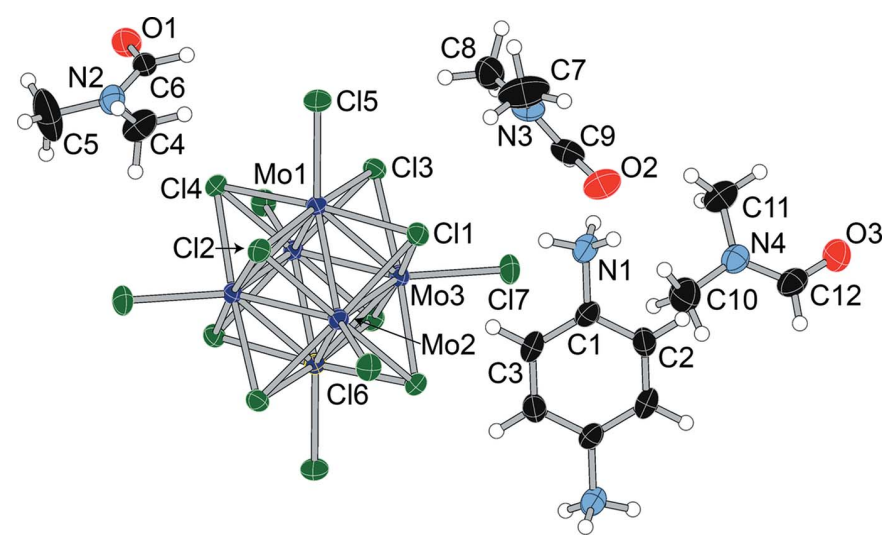

Figure 3

Displacement ellipsoid plot and atom-numbering scheme for (II), with ellipsoids drawn at the $50 \%$ probability level. The minor component of the disordered $p$-phenylenediammonium cation is not shown for clarity. 
Table 1

Hydrogen-bond geometry $\left(\AA{ }^{\circ}\right)$ for $(\mathbf{I})$.

\begin{tabular}{lllll}
\hline$D-\mathrm{H} \cdots A$ & $D-\mathrm{H}$ & $\mathrm{H} \cdots A$ & $D \cdots A$ & $D-\mathrm{H} \cdots A$ \\
\hline $\mathrm{N} 1-\mathrm{H} 1 A \cdots \mathrm{O} 2^{\mathrm{i}}$ & $0.89(3)$ & $2.01(3)$ & $2.827(3)$ & $152(2)$ \\
$\mathrm{N} 1-\mathrm{H} 1 B \cdots \mathrm{O} 2^{\mathrm{ii}}$ & $0.91(3)$ & $1.94(3)$ & $2.833(3)$ & $168(3)$ \\
$\mathrm{N} 1-\mathrm{H} 1 C \cdots{ }^{i i i}$ & $0.91(3)$ & $1.82(3)$ & $2.715(3)$ & $166(3)$ \\
\hline
\end{tabular}

Symmetry codes: (i) $-x+1,-y+1,-z$; (ii) $x-1, y, z$; (iii) $x, y, z-1$.

Table 2

Hydrogen-bond geometry $\left(\AA,^{\circ}\right)$ for $(\mathbf{I I})$.

\begin{tabular}{lllll}
\hline$D-\mathrm{H} \cdots A$ & $D-\mathrm{H}$ & $\mathrm{H} \cdots A$ & $D \cdots A$ & $D-\mathrm{H} \cdots A$ \\
\hline $\mathrm{N} 1-\mathrm{H} 1 A \cdots \mathrm{O} 2^{\mathrm{i}}$ & $0.92(2)$ & $1.76(2)$ & $2.672(4)$ & $171(4)$ \\
$\mathrm{N} 1-\mathrm{H} 1 B \cdots 3^{\text {ii }}$ & $0.93(2)$ & $1.79(2)$ & $2.710(4)$ & $173(4)$ \\
$\mathrm{N} 1-\mathrm{H} 1 C \cdots \mathrm{O} 1^{\text {iii }}$ & $0.92(2)$ & $1.81(2)$ & $2.727(4)$ & $175(4)$ \\
\hline $\begin{array}{l}\text { Symmetry codes: } \\
-x+1,-y+1,-z+1 .\end{array}$ & (i) $\quad x, y+1, z-1 ;$ & (ii) & $-x+2,-y+1,-z ;$ & (iii)
\end{tabular}

over two positions (rotation of $70.6^{\circ}$ about the $\mathrm{N}-\mathrm{N}$ axis), with a refined occupancy of 0.918 (4) for the primary orientation. The structure with the atom-numbering scheme is shown in Fig. 3.

The asymmetric unit of Schiff base salt (III) contains half a cluster unit, half a Schiff base cation, and two independent acetone molecules. The structure with the atom-numbering scheme is shown in Fig. 4. One acetone molecule is disordered over an inversion center. The Schiff base cation, presumably formed from the reaction between a $p$-phenylenediammonium cation and two acetone molecules, shows strong similarities to the cation found in the bismuthate structure reported by Shestimerova et al. (2018).

For comparison, a dicationic salt incapable of conventional hydrogen bonding (methyl viologen) was prepared and structurally characterized. The asymmetric unit of (IV), as in

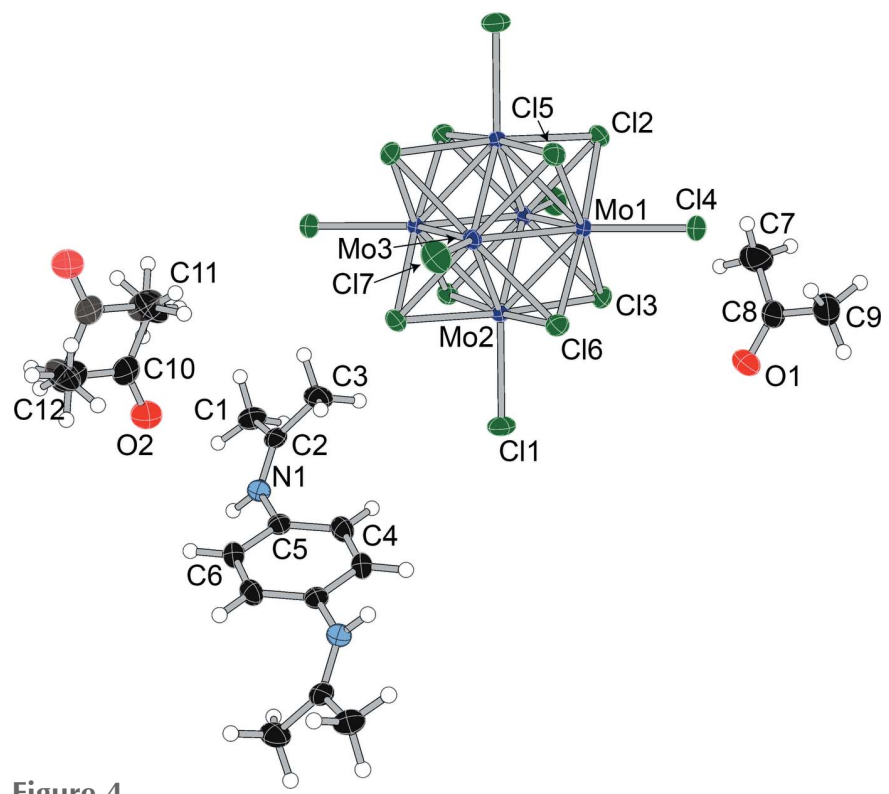

Figure 4

Displacement ellipsoid plot and atom-numbering scheme for (III), with ellipsoids drawn at the $50 \%$ probability level.
Table 3

Hydrogen-bond geometry $\left(\AA,^{\circ}\right)$ for (III).

\begin{tabular}{lllll}
\hline$D-\mathrm{H} \cdots A$ & $D-\mathrm{H}$ & $\mathrm{H} \cdots A$ & $D \cdots A$ & $D-\mathrm{H} \cdots A$ \\
\hline $\mathrm{N} 1-\mathrm{H} 1 \cdots \mathrm{O} 1^{\mathrm{i}}$ & $0.87(2)$ & $1.93(2)$ & $2.791(4)$ & $172(3)$ \\
\hline
\end{tabular}

Symmetry code: (i) $x, y-1, z$.

Table 4

Hydrogen-bond geometry $\left(\AA,{ }^{\circ}\right)$ for $(\mathbf{I V})$.

\begin{tabular}{lllll}
\hline$D-\mathrm{H} \cdots A$ & $D-\mathrm{H}$ & $\mathrm{H} \cdots A$ & $D \cdots A$ & $D-\mathrm{H} \cdots A$ \\
\hline $\mathrm{C} 5-\mathrm{H} 5 \cdots \mathrm{O} 1^{\mathrm{i}}$ & 0.95 & 2.23 & $3.063(4)$ & 145 \\
$\mathrm{C} 6-\mathrm{H} 6 C \cdots \mathrm{O} 2^{\mathrm{ii}}$ & 0.98 & 2.31 & $3.088(4)$ & 136
\end{tabular}

Symmetry codes: (i) $x, y+1, z$; (ii) $-x,-y+2,-z+1$.

the other structures, contains half of the cluster unit, half of the methyl viologen dication, and two independent DMF molecules. The structure with the atom-numbering scheme is shown in Fig. 5.

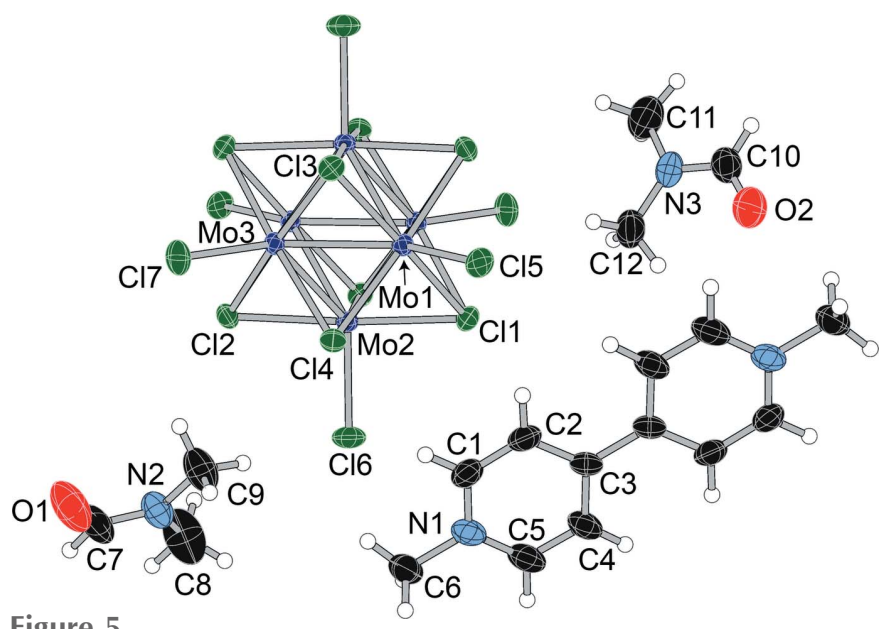

Figure 5

Displacement ellipsoid plot and atom-numbering scheme for (IV), with ellipsoids drawn at the $50 \%$ probability level.

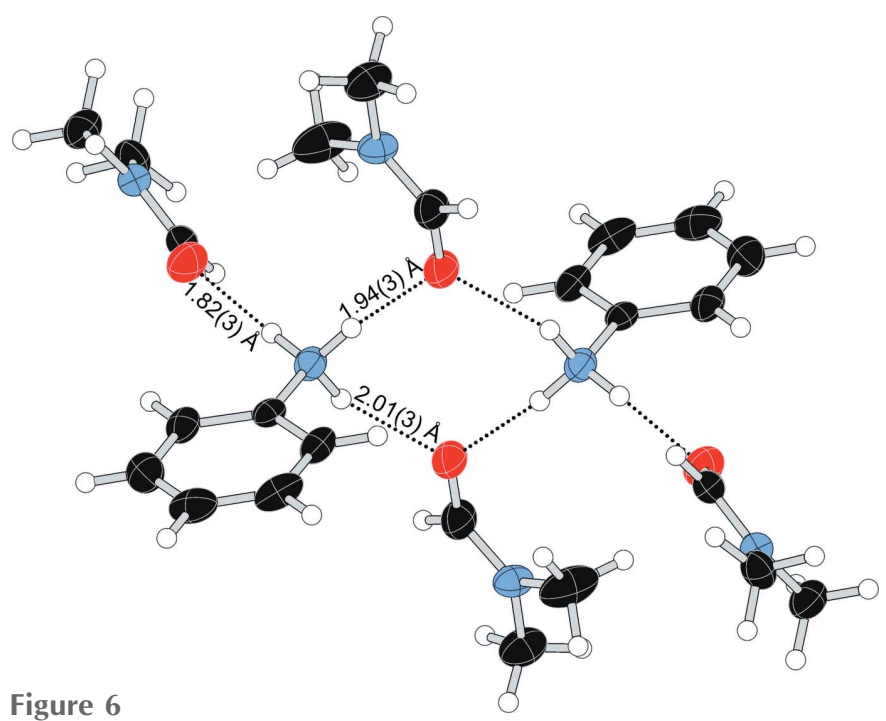

The cationic hydrogen-bonded dimer formed by anilinium cations and DMF molecules in (I). 


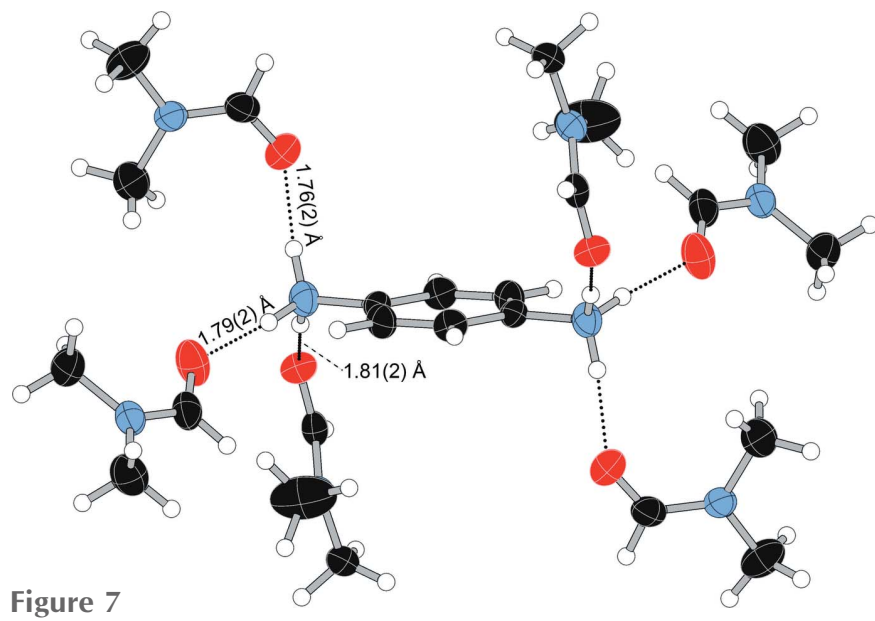

Hydrogen bonding in the DMF-solvated $p$-phenylenediammonium dication in (II). The minor component of the disordered $p$-phenylenediammonium cation is not shown for clarity.

\section{Hydrogen-bonding analysis}

In compound (I), the anilinium cation and DMF molecules form a cyclic $R_{4}^{2}(8)$ hydrogen-bonded motif centered on a crystallographic inversion center, with an additional DMF forming a $D(2)$ interaction, as illustrated in Fig. 6. Although similar to some motifs discussed by Loehlin \& Okasako (2007), the hydrogen-bonding network in (I) does not represent an example of saturated hydrogen bonding, as one DMF molecule has an additional lone pair that is not involved in hydrogen bonding (Table 1). The DMF molecules in compound (II) form three unique $D(2)$ interactions with the three $\mathrm{N}-\mathrm{H}$ bonds on each end of the $p$-phenylenediammonium cations, as shown in Fig. 7 (Table 2). In compound (III), one acetone molecule forms a hydrogen-bonding interaction with the $\mathrm{N}-\mathrm{H}$ group of the Schiff base, as shown in Fig. 8 (Table 3).

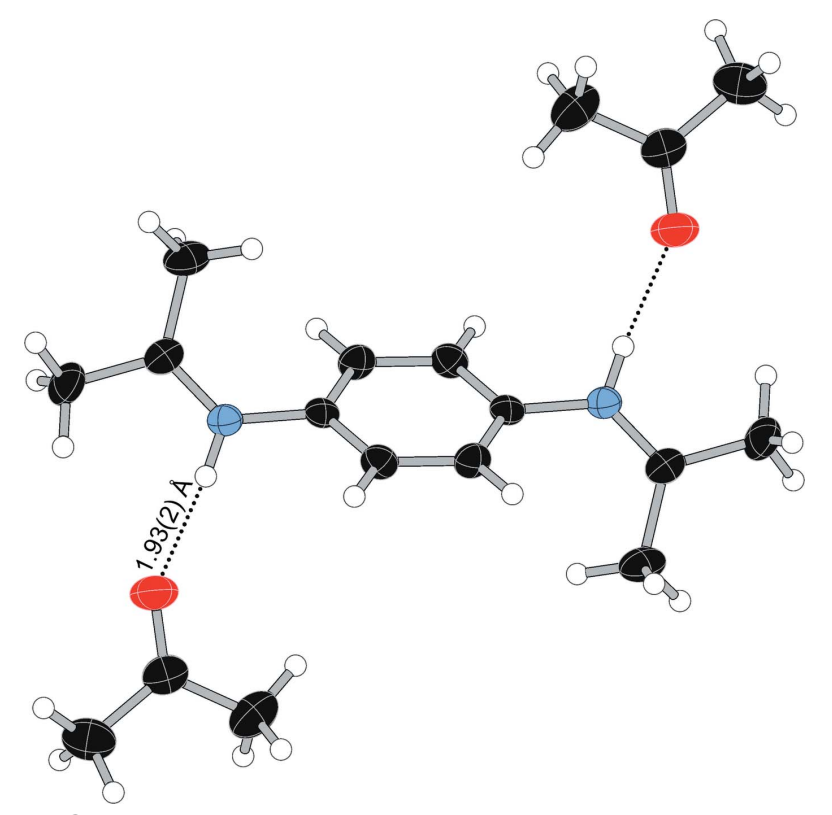

Figure 8

Hydrogen bonding in the acetone-solvated Schiff base dication in (III).

In spite of the lack of conventional hydrogen bonding in compound (IV), the methyl viologen cation forms several C$\mathrm{H}$. O O contacts, with the $\mathrm{O}$ atoms of the two independent DMF molecules forming close contacts with the $\mathrm{H}$ atoms of the aromatic ring $(\mathrm{O} \cdots \mathrm{H}=2.23 \AA)$ and the methyl group $(\mathrm{O} \cdot \mathrm{H}=2.31 \AA$ ) (Table 4).

Analysis of the hydrogen bonding and close contacts via Hirshfeld surfaces and fingerprint plots was conducted using CrystalExplorer (Spackman \& Jayatilaka, 2009) and the results are shown in Fig. 9. Compound (II) has the strongest hydrogen-bonding interactions, with similar, but slightly weaker, interactions for (I) and (III). All four compounds show very similar $\mathrm{H}$ (cation) $\cdots \mathrm{Cl}$ (cluster anion) interactions.

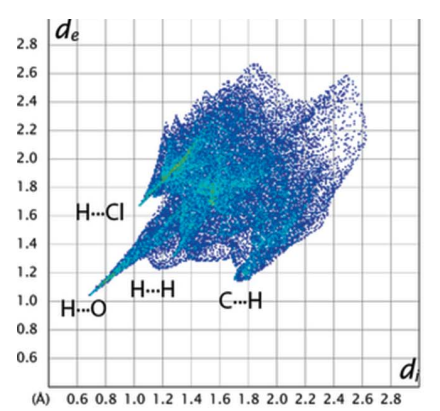

(I)

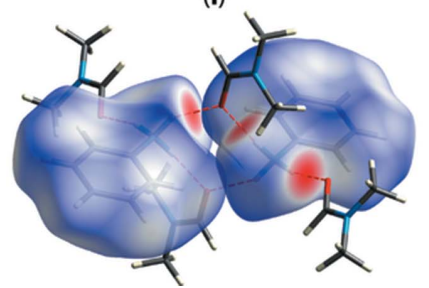

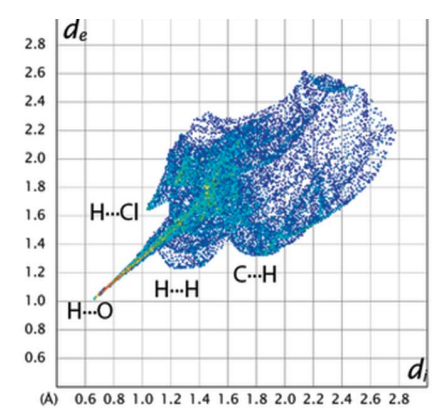

(II)

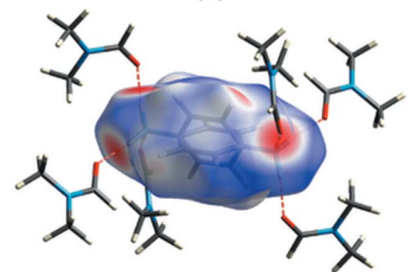

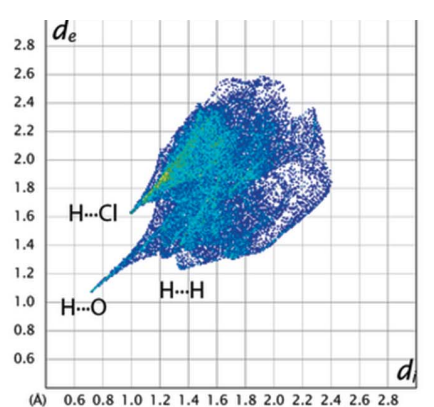

(III)

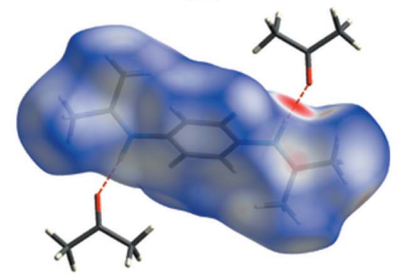

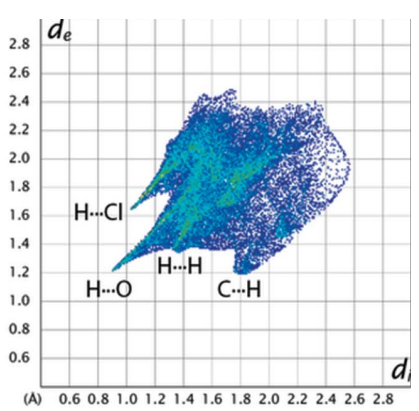

(IV)

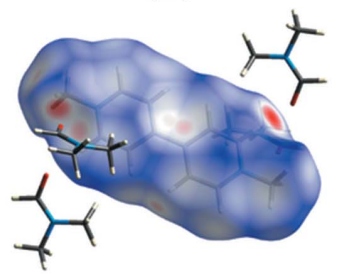

Figure 9

Fingerprint plots and Hirshfeld surfaces for (I)-(IV). For (II), only the major component of the disordered $p$-phenylenediammonium cation was included in the generation of the fingerprint plot. 
Table 5

Experimental details.

(I)

Crystal data

Chemical formula

$M_{\mathrm{r}}$

Crystal system, space group

Temperature (K)

$a, b, c(\AA)$

$\alpha, \beta, \gamma\left(^{\circ}\right)$

$V\left(\AA^{3}\right)$

Radiation type

$\mu\left(\mathrm{mm}^{-1}\right)$

Crystal size (mm)

Data collection

Diffractometer

Absorption correction

$T_{\min }, T_{\max }$

No. of measured, independent and observed $[I>$ $2 \sigma(I)]$ reflections

$R_{\text {int }}$

$(\sin \theta / \lambda)_{\max }\left(\AA^{-1}\right)$

0.026

0.597

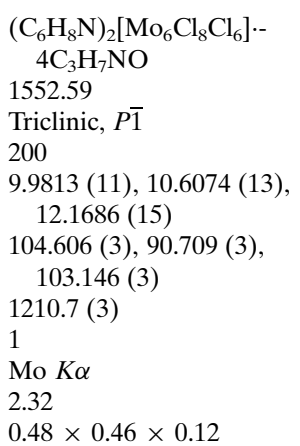

Bruker SMART X2S

benchtop

Multi-scan (SADABS

Bruker, 2012)

$0.498,0.745$

$11709,4245,3834$

Refinement

$R\left[F^{2}>2 \sigma\left(F^{2}\right)\right], w R\left(F^{2}\right), S$

No. of reflections

No. of parameters

No. of restraints

$\mathrm{H}$-atom treatment

$\Delta \rho_{\max }, \Delta \rho_{\min }\left(\mathrm{e} \AA^{-3}\right)$
(II)

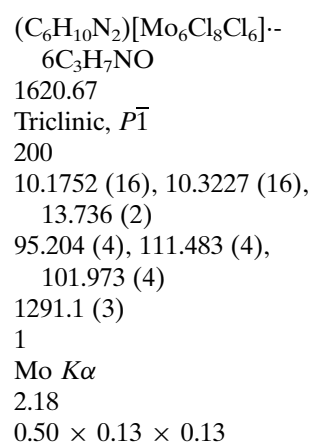

\section{Bruker SMART X2S}

benchtop

Multi-scan ( $S A D A B S$;

Bruker, 2012)

$0.552,0.745$

$12459,4504,3666$

0.035

0.595

$0.026,0.062,1.03$
4504
285
144
H atoms treated by a
$\quad$ mixture of independent
and constrained refine-
ment
$0.66,-0.56$

(III)

$\left(\mathrm{C}_{12} \mathrm{H}_{18} \mathrm{~N}_{2}\right)\left[\mathrm{Mo}_{6} \mathrm{Cl}_{8} \mathrm{Cl}_{6}\right] \cdot-$
$3 \mathrm{C}_{3} \mathrm{H}_{6} \mathrm{O}$
1436.46
Triclinic, $P \overline{1}$
200
$9.451(2), 11.236(3)$,
$\quad 11.712(3)$
$\quad 64.933(6), 71.174(6)$,
$\quad 75.440(6)$
$1056.7(5)$
1
Mo Ko
2.64
$0.55 \times 0.33 \times 0.20$

Bruker SMART X2S benchtop

Multi-scan (SADABS;

Bruker, 2012)

$0.490,0.745$

$10036,3692,3220$

0.030

0.598

$0.025,0.068,1.05$
3692
235
13
$\mathrm{H}$ atoms treated by a
$\quad$ mixture of independent
$\quad$ and constrained refine-
$\quad$ ment
$0.96,-0.82$

(IV)

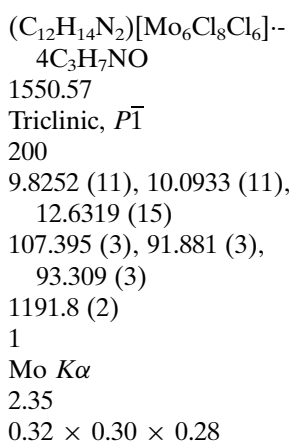

Bruker SMART X2S
$\quad$ benchtop
Multi-scan (SADABS;
$\quad$ Bruker, 2012)
$0.815,1.000$
$11498,4187,3743$

0.024
0.597

$0.020,0.049,1.02$
4187
250
0
H-atom parameters
$\quad$ constrained

$0.42,-0.44$

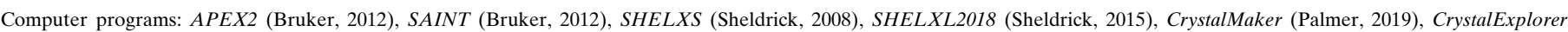
(Spackman \& Jayatilaka, 2009), OLEX2 (Dolomanov et al., 2009) and publCIF (Westrip, 2010).

The $\mathrm{C}-\mathrm{H} \cdots \mathrm{O}$ contacts in (IV), especially with the aromatic $\mathrm{C}-\mathrm{H}$ group of the methyl viologen, can be clearly identified on the Hirshfeld surface.

\section{Database survey}

Interest in molybdenum(II) halide clusters and related compounds have led to numerous structural studies, with 45 entries in the Cambridge Structural Database (CSD, Version 5.40; Groom et al., 2016) containing the $\left[\mathrm{Mo}_{6} \mathrm{Cl}_{14}\right]^{2-}$ dianion and almost 200 structures containing the $\left[\mathrm{Mo}_{6} X_{8}\right]^{4+}$ core. Similarly, one can find over 50 structures in the Inorganic Crystal Structure Database (ICSD, Version 4.2.0; Hellenbrandt, 2004) containing the same molybdenum halide core structure. The structures of the $\left[\mathrm{Mo}_{6} \mathrm{Cl}_{14}\right]^{2-}$ cluster anions in this study are unremarkable and do not differ significantly from previous studies.

The anilinium cluster dihydrate structure published by Flemström (2003) has some similarities to (I). In that structure, the three $\mathrm{N}-\mathrm{H}$ bonds of the anilinium cation serve as hydrogen-bond donors to one water molecule (hydrate) and two terminal $\mathrm{Cl}$ atoms on two discrete cluster anions. The $\mathrm{N}-$ $\mathrm{H}$. C Cl interactions create $C_{4}^{4}(15)$ chains. The water molecules create $R_{4}^{4}(14)$ rings involving two water molecules and two cluster units, as well as $C_{2}^{2}(8)$ and $C_{2}^{2}(7)$ chains.

While DMF-solvated ammonium salts appear to be relatively uncommon, a series of molybdenum halide cluster salts have been prepared with dimethylformamide-coordinated metal cations serving as the counter-cation (Khutornoi et al., 2002; Kozhomuratova et al., 2007; Liu et al., 2006). The complexes prepared and characterized include the $\left[\mathrm{Mo}_{6} \mathrm{Cl}_{8} \mathrm{Cl}_{6}\right]^{2-}$, $\left[\mathrm{Mo}_{6} \mathrm{Br}_{8} \mathrm{Cl}_{6}\right]^{2-}$, and $\left[\mathrm{Mo}_{6} \mathrm{Br}_{8}(\mathrm{NCS})_{6}\right]^{2-}$ cluster anions as salts with $[M(\mathrm{DMF})]^{2+}$ cations, where $M=\mathrm{Ca}^{2+}, \mathrm{Mn}^{2+}$, and $\mathrm{Co}^{2+} . \mathrm{A}$ similar set of rhenium chalcogenide cluster salts with DMFsolvated calcium and a series of lanthanides has been prepared by Perruchas et al. (2002) and Yarovoi et al. (2006).

A separate search of the CSD for structures with similar hydrogen-bonded networks containing anilinium and $p$-phenylenediammonium cations yielded a large number of hits due to their propensity for forming significant hydrogenbonding networks. In the structure of anilinium dihydrogen phosphate (Kaman et al., 2012), each of the three independent 
ammonium groups forms four different hydrogen bonds to the $\mathrm{O}$ atoms of nearby dihydrogen phosphate moieties. A very similar set of hydrogen-bonding interactions and layered organic/inorganic structural arrangements are found in the structures of $p$-phenylenediammonium bis(dihydrogen phosphate) (Mrad et al., 2006a) and $p$-phenylenediammonium dihydrogen diphosphate (Mrad et al., 2006b). While less closely related to the current report, the structure of $p$-phenylenediammonium tetrachloridozincate(II) (Bringley \& Rajeswaran, 2006) also displays alternating organic and inorganic layers and strong hydrogen bonding between the tetrachloridozinc(II) anions and the $p$-phenylenediammonium cations.

A dimethyl sulfoxide (DMSO)-solvated $p$-phenylenediammonium salt of an iodidobismuthate reported by Shestimerova et al. (2018) displays strong structural similarities to (II) in the way the DMSO solvates the $p$-phenylenediammonium cation. Three unique DMSO molecules also form $D(2)$ interactions with each end of the $p$-phenylenediammonium. One of the three DMSO molecules simultaneously coordinates to one of the $\mathrm{Bi}$ atoms.

\section{Synthesis and crystallization}

All reagents were used as received from the manufacturer.

\subsection{Cluster synthesis, metathesis, and crystallization of (I), (II), and (IV)}

The hydronium salt of the $\left[\mathrm{Mo}_{6} \mathrm{Cl}_{8} \mathrm{Cl}_{6}\right]^{2-}$ anion was prepared by the method of Hay et al. (2004) and then metathesized to the appropriate ammonium salt by combining an ethanolic solution of $\left(\mathrm{H}_{3} \mathrm{O}\right)_{2}\left[\mathrm{Mo}_{6} \mathrm{Cl}_{8} \mathrm{Cl}_{6}\right] \cdot 6 \mathrm{H}_{2} \mathrm{O}$ with a slight stoichiometric excess ( $\sim 2.5$ times $)$ of the respective ammonium chloride salt (anilinium chloride, $p$-phenylenediamine hydrochloride, and methyl viologen dichloride). The brightyellow precipitate that formed was isolated by filtration and the product was recrystallized by vapor diffusion of diethyl ether into a dimethylformamide solution of the cluster salt.

\subsection{Synthesis and crystallization of Schiff base salt (III)}

The cluster in compound (III) was prepared and metathesized to the diammonium salt via the same procedure as above using the $p$-phenylenediammonium chloride to isolate a yellow precipitate. The salt was then redissolved in acetone and allowed to evaporate. The acetone inadvertently formed a Schiff base dication in a reaction with the $p$-phenylenediammonium cation (Kolb \& Bahadir, 1994).

\section{Refinement}

Crystal data, data collection, and structure refinement details are summarized in Table 5. All $\mathrm{H}$ atoms were located in a difference map. All carbon-bonded $\mathrm{H}$ atoms were placed in idealized positions using a riding model, with aromatic and amide $\mathrm{C}-\mathrm{H}=0.95 \AA$ and methyl $\mathrm{C}-\mathrm{H}=0.98 \AA$, and with $U_{\text {iso }}(\mathrm{H})=1.2 U_{\text {eq }}(\mathrm{C})$ (aromatic and amide) or $U_{\text {iso }}(\mathrm{H})=$ $1.5 U_{\text {eq }}(\mathrm{C})$ (methyl). The positions of all $\mathrm{H}$ atoms bonded to $\mathrm{N}$ atoms were refined with $\mathrm{N}-\mathrm{H}$ distances restrained to 0.91 (2) $\left(\mathrm{NH}_{3}\right)$ or $0.88(2) \AA$ (Schiff base), and with $U_{\text {iso }}(\mathrm{H})=$ $1.5 U_{\text {eq }}(\mathrm{N})$.

All four structures were refined in the space group $P \overline{1}$ and the $\left[\mathrm{Mo}_{6} \mathrm{Cl}_{14}\right]^{2-}$ dianion sits on an inversion center in every case. The dications in (II), (III), and (IV) are also each located on an inversion center. The $p$-phenylenediammonium cation in (II) is disordered over two orientations with an occupancy of 0.918 (4) for the major component. One of the two acetone molecules in (III) is disordered over an inversion center.

\section{Acknowledgements}

This work was supported in part by the National Science Foundation. The authors thank H. Kaur and A. Maldonado for initial work on this project.

\section{Funding information}

Funding for this research was provided by: National Science Foundation, Division Of Undergraduate Education (grant No. 0942850 to DHJ).

\section{References}

Akagi, S., Fujii, S. \& Kitamura, N. (2018). Dalton Trans. 47, 11311139.

Bringley, J. F. \& Rajeswaran, M. (2006). Acta Cryst. E62, m1304m1305.

Brozdowska, A. \& Chojnacki, J. (2017). Acta Cryst. B73, 507-518.

Bruker (2012). APEX2, SAINT, and SADABS. Bruker AXS Inc., Madison, Wisconsin, USA.

Brychczynska, M., Davey, R. J. \& Pidcock, E. (2012). CrystEngComm, 14, 1479-1484.

Dolomanov, O. V., Bourhis, L. J., Gildea, R. J., Howard, J. A. K. \& Puschmann, H. (2009). J. Appl. Cryst. 42, 339-341.

Flemström, A. (2003). Acta Cryst. E59, m162-m164.

Gilli, G. \& Gilli, P. (2009). The Nature of the Hydrogen Bond: Outline of a Comprehensive Hydrogen Bond Theory. Oxford University Press.

Groom, C. R., Bruno, I. J., Lightfoot, M. P. \& Ward, S. C. (2016). Acta Cryst. B72, 171-179.

Groot, J. de, Gojdas, K., Unruh, D. K. \& Forbes, T. Z. (2014). Cryst. Growth Des. 14, 1357-1365.

Gurbanov, A. V., Mahmudov, K. T., Kopylovich, M. N., Guedes da Silva, F. M., Sutradhar, M., Guseinov, F. I., Zubkov, F. I., Maharramov, A. M. \& Pombeiro, A. J. L. (2017). Dyes Pigments, 138, 107-111.

Hay, D. N., Adams, J. A., Carpenter, J., DeVries, S. L., Domyancich, J., Dumser, B., Goldsmith, S., Kruse, M. A., Leone, A., MoussaviHarami, F., O’Brien, J. A., Pfaffly, J. R., Sylves, M., Taravati, P., Thomas, J. L., Tiernan, B. \& Messerle, L. (2004). Inorg. Chim. Acta, 357, 644-648.

Hellenbrandt, M. (2004). Crystallogr. Rev. 10, 17-22.

Kaman, O., Smrcok, L., Gyepes, R. \& Havlícek, D. (2012). Acta Cryst. C68, o57-060.

Khutornoi, V. A., Naumov, N. G., Mironov, Y. V., Oeckler, O., Simon, A. \& Fedorov, V. E. (2002). Russ. J. Coord. Chem. 28, 183-190.

Kolb, M. \& Bahadir, M. (1994). J. Chromatogr. A, 685, 189-194.

Kozhomuratova, Z. S., Mironov, Y. V., Shestopalov, M. A., Gaifulin, Y. M., Kurat'eva, N. V., Uskov, E. M. \& Fedorov, V. E. (2007). Russ. J. Coord. Chem. 33, 1-6.

Liu, X., Cai, L.-Z., Guo, C.-C., Li, Q. \& Huang, J.-S. (2006). Jiegou Hиахие, 25, 90-94.

Loehlin, J. H. \& Okasako, E. L. N. (2007). Acta Cryst. B63, 132-141. 
Maverick, A. W., Najdzionek, J. S., MacKenzie, D., Nocera, D. G. \& Gray, H. B. (1983). J. Am. Chem. Soc. 105, 1878-1882.

Mikhailov, M. A., Brylev, K. A., Abramov, P. A., Sakuda, E., Akagi, S., Ito, A., Kitamura, N. \& Sokolov, M. N. (2016). Inorg. Chem. 55, 8437-8445.

Mrad, M. L., Nasr, C. B. \& Rzaigui, M. (2006a). Mater. Res. Bull. 41, 1287-1294.

Mrad, M. L., Nasr, C. B., Rzaigui, M. \& Lefebvre, F. (2006b). Phosphorus Sulfur Silicon, 181, 1625-1635.

Palmer, D. C. (2019). CrystalMaker. CrystalMaker Software Ltd, Begbroke, Oxfordshire, England.

Perruchas, S., Simon, F., Uriel, S., Avarvari, N., Boubekeur, K. \& Batail, P. (2002). J. Organomet. Chem. 643-644, 301-306.

Pop, L., Hadade, N. D., van der Lee, A., Barboiu, M., Grosu, I. \& Legrand, Y.-M. (2016). Cryst. Growth Des. 16, 3271-3278.
Saito, N., Lemoine, P., Dumait, N., Amela-Cortes, M., Paofai, S., Roisnel, T., Nassif, V., Grasset, F., Wada, Y., Ohashi, N. \& Cordier, S. (2017). J. Cluster Sci. 28, 773-798.

Sheldrick, G. M. (2008). Acta Cryst. A64, 112-122.

Sheldrick, G. M. (2015). Acta Cryst. A71, 3-8.

Shestimerova, T. A., Golubev, N. A., Mironov, A. V., Bykov, M. A. \& Shevelkov, A. V. (2018). Russ. Chem. Bull. 67, 1212-1219.

Spackman, M. A. \& Jayatilaka, D. (2009). CrystEngComm, 11, 19-32.

Ward, M. D. (2009). Molecular Networks, Vol. 132, edited by M. W. Hosseini, pp. 1-23. Berlin, Heidelberg: Springer.

Westrip, S. P. (2010). J. Appl. Cryst. 43, 920-925.

Yarovoi, S. S., Mironov, Yu. V., Solodovnikov, S. F., Solodovnikova, Z. A., Naumov, D. Yu. \& Fedorov, V. E. (2006). Russ. J. Coord. Chem. 32, 712-722.

Zick, P. L. \& Geiger, D. K. (2018). Acta Cryst. C74, 1725-1731. 


\section{supporting information}

Acta Cryst. (2019). E75, 1705-1711 [https://doi.org/10.1107/S205698901901380X]

\section{Crystal structures and hydrogen-bonding analysis of a series of solvated ammonium salts of molybdenum(II) chloride clusters}

\section{Dean H. Johnston and Ikponmwosa Agho}

Computing details

For all structures, data collection: APEX2 (Bruker, 2012); cell refinement: SAINT (Bruker, 2012); data reduction: SAINT (Bruker, 2012); program(s) used to solve structure: SHELXS (Sheldrick, 2008); program(s) used to refine structure: SHELXL2018 (Sheldrick, 2015); molecular graphics: CrystalMaker (Palmer, 2019) and CrystalExplorer (Spackman \& Jayatilaka, 2009); software used to prepare material for publication: OLEX2 (Dolomanov et al., 2009) and publCIF (Westrip, 2010).

Bis(anilinium) octa- $\mu_{3}$-chlorido-hexachlorido-octahedro-hexamolybdate $N, N$-dimethylformamide tetrasolvate

Crystal data

$\left(\mathrm{C}_{6} \mathrm{H}_{8} \mathrm{~N}\right)_{2}\left[\mathrm{Mo}_{6} \mathrm{Cl}_{8} \mathrm{Cl}_{6}\right] \cdot 4 \mathrm{C}_{3} \mathrm{H}_{7} \mathrm{NO}$

$M_{r}=1552.59$

Triclinic, $P \overline{1}$

$a=9.9813(11) \AA$

$b=10.6074(13) \AA$

$c=12.1686(15) \AA$

$\alpha=104.606(3)^{\circ}$

$\beta=90.709(3)^{\circ}$

$\gamma=103.146(3)^{\circ}$

$V=1210.7(3) \AA^{3}$

\section{Data collection}

Bruker SMART X2S benchtop diffractometer

Radiation source: sealed microfocus source, XOS X-beam microfocus source

Graphite monochromator

Detector resolution: 8.3330 pixels $\mathrm{mm}^{-1}$

$\varphi$ and $\omega$ scans

Absorption correction: multi-scan

(SADABS; Bruker, 2012)

\section{Refinement}

Refinement on $F^{2}$

Least-squares matrix: full

$R\left[F^{2}>2 \sigma\left(F^{2}\right)\right]=0.019$

$w R\left(F^{2}\right)=0.046$

$S=1.07$
$Z=1$

$F(000)=752$

$D_{\mathrm{x}}=2.129 \mathrm{Mg} \mathrm{m}^{-3}$

Mo $K \alpha$ radiation, $\lambda=0.71073 \AA$

Cell parameters from 7006 reflections

$\theta=2.3-25.1^{\circ}$

$\mu=2.32 \mathrm{~mm}^{-1}$

$T=200 \mathrm{~K}$

Plate, clear orangish yellow

$0.48 \times 0.46 \times 0.12 \mathrm{~mm}$

$T_{\min }=0.498, T_{\max }=0.745$

11709 measured reflections

4245 independent reflections

3834 reflections with $I>2 \sigma(I)$

$R_{\text {int }}=0.026$

$\theta_{\text {max }}=25.1^{\circ}, \theta_{\min }=2.3^{\circ}$

$h=-11 \rightarrow 11$

$k=-12 \rightarrow 12$

$l=-14 \rightarrow 14$

4245 reflections

258 parameters

0 restraints

Primary atom site location: structure-invariant direct methods 
Hydrogen site location: mixed

$\mathrm{H}$ atoms treated by a mixture of independent and constrained refinement

$w=1 /\left[\sigma^{2}\left(F_{\mathrm{o}}^{2}\right)+(0.0123 P)^{2}+0.5808 P\right]$

where $P=\left(F_{\mathrm{o}}{ }^{2}+2 F_{\mathrm{c}}{ }^{2}\right) / 3$

$(\Delta / \sigma)_{\max }=0.001$
$\Delta \rho_{\max }=0.36 \mathrm{e} \AA^{-3}$

$\Delta \rho_{\min }=-0.54 \mathrm{e} \AA^{-3}$

Extinction correction: SHELXL2018

(Sheldrick, 2015),

$\mathrm{Fc}^{*}=\mathrm{kFc}\left[1+0.001 \times \mathrm{xc}^{2} \lambda^{3} / \sin (2 \theta)\right]^{-1 / 4}$

Extinction coefficient: 0.0096 (3)

Special details

Geometry. All esds (except the esd in the dihedral angle between two 1.s. planes) are estimated using the full covariance matrix. The cell esds are taken into account individually in the estimation of esds in distances, angles and torsion angles; correlations between esds in cell parameters are only used when they are defined by crystal symmetry. An approximate (isotropic) treatment of cell esds is used for estimating esds involving l.s. planes.

Fractional atomic coordinates and isotropic or equivalent isotropic displacement parameters $\left(\hat{A}^{2}\right)$

\begin{tabular}{|c|c|c|c|c|}
\hline & $x$ & $y$ & $z$ & $U_{\text {iso }} * / U_{\text {eq }}$ \\
\hline Mo1 & $0.33000(2)$ & $0.50142(2)$ & $0.43962(2)$ & $0.01755(7)$ \\
\hline Mo2 & $0.42505(2)$ & $0.33816(2)$ & $0.52988(2)$ & $0.01753(7)$ \\
\hline Mo3 & $0.45872(2)$ & $0.58737(2)$ & $0.64151(2)$ & $0.01766(7)$ \\
\hline $\mathrm{Cl1}$ & $0.30660(6)$ & $0.26453(5)$ & $0.33717(5)$ & $0.02208(12)$ \\
\hline $\mathrm{Cl} 2$ & $0.44848(6)$ & $0.57046(5)$ & $0.27978(5)$ & $0.02298(13)$ \\
\hline $\mathrm{Cl} 3$ & $0.37196(6)$ & $0.73926(5)$ & $0.55008(5)$ & $0.02352(13)$ \\
\hline $\mathrm{Cl} 4$ & $0.22876(5)$ & $0.43149(5)$ & $0.60532(5)$ & $0.02318(13)$ \\
\hline $\mathrm{Cl} 5$ & $0.32774(6)$ & $0.12583(6)$ & $0.57003(5)$ & $0.03234(15)$ \\
\hline $\mathrm{Cl} 6$ & $0.39836(6)$ & $0.69501(6)$ & $0.82888(5)$ & $0.03331(15)$ \\
\hline $\mathrm{Cl} 7$ & $0.10839(6)$ & $0.50315(6)$ & $0.35713(5)$ & $0.03118(14)$ \\
\hline N1 & $0.1138(2)$ & $0.6861(2)$ & -0.02394 (19) & $0.0291(5)$ \\
\hline $\mathrm{H} 1 \mathrm{~A}$ & $0.144(3)$ & $0.612(3)$ & $-0.048(2)$ & $0.044^{*}$ \\
\hline H1B & $0.026(3)$ & $0.651(3)$ & $-0.010(2)$ & $0.044^{*}$ \\
\hline $\mathrm{H} 1 \mathrm{C}$ & $0.111(3)$ & $0.732(3)$ & $-0.078(2)$ & $0.044 *$ \\
\hline $\mathrm{C} 1$ & $0.1999(2)$ & $0.7715(2)$ & $0.0778(2)$ & $0.0262(5)$ \\
\hline $\mathrm{C} 2$ & $0.1890(3)$ & $0.7327(3)$ & $0.1777(2)$ & $0.0352(6)$ \\
\hline $\mathrm{H} 2$ & 0.125907 & 0.652029 & 0.180955 & $0.042 *$ \\
\hline $\mathrm{C} 3$ & $0.2713(3)$ & $0.8128(3)$ & $0.2728(2)$ & $0.0439(7)$ \\
\hline H3 & 0.264848 & 0.787222 & 0.342295 & $0.053 *$ \\
\hline $\mathrm{C} 4$ & $0.3621(3)$ & $0.9288(3)$ & 0.2678 & $0.0454(7)$ \\
\hline $\mathrm{H} 4$ & 0.418475 & 0.983410 & 0.333788 & $0.055^{*}$ \\
\hline $\mathrm{C} 5$ & $0.2894(3)$ & $0.8879(2)$ & $0.0709(2)$ & $0.0326(6)$ \\
\hline H5 & 0.294613 & 0.913873 & 0.001580 & $0.039^{*}$ \\
\hline C6 & 0.3718 & $0.9666(3)$ & $0.1671(2)$ & $0.0411(7)$ \\
\hline H6 & 0.435330 & 1.046973 & 0.163742 & $0.049^{*}$ \\
\hline $\mathrm{O} 1$ & $0.0921(2)$ & $0.85206(19)$ & $0.84303(16)$ & $0.0431(5)$ \\
\hline $\mathrm{N} 2$ & $0.0472(2)$ & $0.88716(19)$ & $0.67193(17)$ & $0.0287(5)$ \\
\hline $\mathrm{C} 7$ & $0.0074(3)$ & $1.0112(3)$ & $0.7195(2)$ & $0.0408(7)$ \\
\hline $\mathrm{H} 7 \mathrm{~A}$ & 0.004355 & 1.025920 & 0.802189 & $0.061^{*}$ \\
\hline H7B & -0.083924 & 1.006277 & 0.685843 & $0.061 *$ \\
\hline $\mathrm{H} 7 \mathrm{C}$ & 0.074846 & 1.085755 & 0.702938 & $0.061^{*}$ \\
\hline $\mathrm{C} 8$ & $0.0860(2)$ & $0.8186(3)$ & $0.7380(2)$ & $0.0325(6)$ \\
\hline H8 & 0.111034 & 0.737608 & 0.702115 & $0.039 *$ \\
\hline
\end{tabular}




$\begin{array}{lllll}\text { C9 } & 0.0410(3) & 0.8403(3) & 0.5486(2) & 0.0364(6) \\ \text { H9A } & 0.057014 & 0.749801 & 0.526886 & 0.055^{*} \\ \text { H9B } & 0.111888 & 0.901063 & 0.519142 & 0.055^{*} \\ \text { H9C } & -0.050269 & 0.838466 & 0.516599 & 0.055^{*} \\ \text { O2 } & 0.85598(19) & 0.57853(18) & 0.05179(17) & 0.0418(5) \\ \text { N3 } & 0.7318(2) & 0.7329(2) & 0.05377(17) & 0.0320(5) \\ \text { C10 } & 0.7698(3) & 0.6417(3) & 0.0921(2) & 0.0397(7) \\ \text { H10 } & 0.727510 & 0.621423 & 0.157083 & 0.048^{*} \\ \text { C11 } & 0.6338(3) & 0.8071(3) & 0.1095(2) & 0.0460(7) \\ \text { H11A } & 0.607218 & 0.779492 & 0.178708 & 0.069^{*} \\ \text { H11B } & 0.551612 & 0.788223 & 0.057583 & 0.069^{*} \\ \text { H11C } & 0.676785 & 0.903485 & 0.129568 & 0.069^{*} \\ \text { C12 } & 0.7879(4) & 0.7684(4) & -0.0466(3) & 0.0621(10) \\ \text { H12A } & 0.866691 & 0.846144 & -0.023497 & 0.093^{*} \\ \text { H12B } & 0.716767 & 0.790546 & -0.089431 & 0.093^{*} \\ \text { H12C } & 0.818232 & 0.692417 & -0.094617 & 0.093^{*}\end{array}$

Atomic displacement parameters $\left(\AA^{2}\right)$

\begin{tabular}{lllllll}
\hline & $U^{11}$ & $U^{22}$ & $U^{33}$ & $U^{12}$ & $U^{13}$ & $U^{23}$ \\
\hline Mo1 & $0.01465(11)$ & $0.01876(11)$ & $0.01888(11)$ & $0.00353(8)$ & $0.00272(8)$ & $0.00463(8)$ \\
Mo2 & $0.01627(11)$ & $0.01691(10)$ & $0.01871(11)$ & $0.00234(8)$ & $0.00337(8)$ & $0.00474(8)$ \\
Mo3 & $0.01646(12)$ & $0.01852(11)$ & $0.01703(11)$ & $0.00379(8)$ & $0.00436(8)$ & $0.00307(8)$ \\
C11 & $0.0205(3)$ & $0.0202(3)$ & $0.0218(3)$ & $0.0011(2)$ & $0.0008(2)$ & $0.0022(2)$ \\
C12 & $0.0233(3)$ & $0.0256(3)$ & $0.0208(3)$ & $0.0045(2)$ & $0.0022(2)$ & $0.0086(2)$ \\
C13 & $0.0231(3)$ & $0.0211(3)$ & $0.0272(3)$ & $0.0086(2)$ & $0.0041(2)$ & $0.0046(2)$ \\
C14 & $0.0169(3)$ & $0.0267(3)$ & $0.0247(3)$ & $0.0029(2)$ & $0.0066(2)$ & $0.0066(2)$ \\
C15 & $0.0345(3)$ & $0.0242(3)$ & $0.0369(3)$ & $-0.0020(3)$ & $0.0024(3)$ & $0.0134(3)$ \\
C16 & $0.0350(4)$ & $0.0359(3)$ & $0.0241(3)$ & $0.0078(3)$ & $0.0112(3)$ & $-0.0010(3)$ \\
C17 & $0.0199(3)$ & $0.0356(3)$ & $0.0397(3)$ & $0.0061(3)$ & $-0.0019(3)$ & $0.0132(3)$ \\
N1 & $0.0306(12)$ & $0.0255(11)$ & $0.0341(12)$ & $0.0094(10)$ & $0.0055(10)$ & $0.0104(10)$ \\
C1 & $0.0273(13)$ & $0.0252(12)$ & $0.0296(13)$ & $0.0132(11)$ & $0.0041(11)$ & $0.0072(10)$ \\
C2 & $0.0437(16)$ & $0.0307(13)$ & $0.0379(15)$ & $0.0162(12)$ & $0.0069(13)$ & $0.0142(12)$ \\
C3 & $0.063(2)$ & $0.0469(17)$ & $0.0309(15)$ & $0.0328(16)$ & $0.0019(14)$ & $0.0097(13)$ \\
C4 & $0.0505(18)$ & $0.0396(16)$ & $0.0433(17)$ & $0.0259(15)$ & $-0.0084(14)$ & $-0.0073(13)$ \\
C5 & $0.0372(15)$ & $0.0263(13)$ & $0.0383(15)$ & $0.0145(11)$ & $0.0117(12)$ & $0.0089(11)$ \\
C6 & $0.0412(17)$ & $0.0277(14)$ & $0.0495(18)$ & $0.0115(12)$ & $0.0063(14)$ & $-0.0016(13)$ \\
O1 & $0.0496(12)$ & $0.0479(11)$ & $0.0392(11)$ & $0.0149(10)$ & $0.0045(9)$ & $0.0219(9)$ \\
N2 & $0.0270(11)$ & $0.0275(11)$ & $0.0326(11)$ & $0.0065(9)$ & $0.0007(9)$ & $0.0094(9)$ \\
C7 & $0.0462(17)$ & $0.0355(15)$ & $0.0465(17)$ & $0.0170(13)$ & $0.0084(14)$ & $0.0144(13)$ \\
C8 & $0.0248(14)$ & $0.0313(13)$ & $0.0428(16)$ & $0.0048(11)$ & $0.0040(12)$ & $0.0140(12)$ \\
C9 & $0.0334(15)$ & $0.0362(14)$ & $0.0332(14)$ & $-0.0008(12)$ & $-0.0040(12)$ & $0.0062(12)$ \\
O2 & $0.0359(11)$ & $0.0345(10)$ & $0.0614(13)$ & $0.0134(9)$ & $0.0149(10)$ & $0.0192(9)$ \\
N3 & $0.0309(12)$ & $0.0407(12)$ & $0.0301(11)$ & $0.0169(10)$ & $0.0066(10)$ & $0.0120(10)$ \\
C10 & $0.0391(16)$ & $0.0390(15)$ & $0.0470(17)$ & $0.0112(13)$ & $0.0145(13)$ & $0.0197(13)$ \\
C11 & $0.0527(19)$ & $0.0527(18)$ & $0.0427(17)$ & $0.0301(15)$ & $0.0149(15)$ & $0.0142(14)$ \\
C12 & $0.073(2)$ & $0.099(3)$ & $0.0438(18)$ & $0.053(2)$ & $0.0248(17)$ & $0.0425(19)$ \\
& & & & & & \\
\hline & & & & & &
\end{tabular}


Geometric parameters $\left(\AA,{ }^{\circ}\right)$

\begin{tabular}{|c|c|c|c|}
\hline $\operatorname{Mol}-\mathrm{Mo}^{\mathrm{i}}$ & $2.6034(4)$ & $\mathrm{C} 3-\mathrm{H} 3$ & 0.9500 \\
\hline Mo1-Mo2 & $2.6051(3)$ & $\mathrm{C} 3-\mathrm{C} 4$ & $1.368(4)$ \\
\hline Mo1-Mo3 ${ }^{\mathrm{i}}$ & $2.6068(3)$ & $\mathrm{C} 4-\mathrm{H} 4$ & 0.9500 \\
\hline Mo1-Mo3 & $2.6032(4)$ & $\mathrm{C} 4-\mathrm{C} 6$ & $1.381(4)$ \\
\hline Mo1-Cl1 & $2.4636(6)$ & $\mathrm{C} 5-\mathrm{H} 5$ & 0.9500 \\
\hline $\mathrm{Mo} 1-\mathrm{Cl} 2$ & $2.4697(6)$ & $\mathrm{C} 5-\mathrm{C} 6$ & $1.383(4)$ \\
\hline $\mathrm{Mo} 1-\mathrm{Cl} 3$ & $2.4782(6)$ & $\mathrm{C} 6-\mathrm{H} 6$ & 0.9500 \\
\hline $\mathrm{Mo1}-\mathrm{Cl} 4$ & $2.4700(6)$ & $\mathrm{O} 1-\mathrm{C} 8$ & $1.234(3)$ \\
\hline $\mathrm{Mo} 1-\mathrm{Cl} 7$ & $2.4235(6)$ & $\mathrm{N} 2-\mathrm{C} 7$ & $1.444(3)$ \\
\hline Mo2-Mo3 & $2.5922(4)$ & $\mathrm{N} 2-\mathrm{C} 8$ & $1.319(3)$ \\
\hline $\mathrm{Mo} 2-\mathrm{Mo}^{\mathrm{i}}$ & $2.6065(3)$ & $\mathrm{N} 2-\mathrm{C} 9$ & $1.453(3)$ \\
\hline $\mathrm{Mo} 2-\mathrm{Cl1}$ & $2.4687(6)$ & $\mathrm{C} 7-\mathrm{H} 7 \mathrm{~A}$ & 0.9800 \\
\hline $\mathrm{Mo} 2-\mathrm{C} 12^{\mathrm{i}}$ & $2.4772(6)$ & $\mathrm{C} 7-\mathrm{H} 7 \mathrm{~B}$ & 0.9800 \\
\hline $\mathrm{Mo} 2-\mathrm{Cl}^{\mathrm{i}}$ & $2.4756(6)$ & $\mathrm{C} 7-\mathrm{H} 7 \mathrm{C}$ & 0.9800 \\
\hline $\mathrm{Mo} 2-\mathrm{Cl} 4$ & $2.4758(6)$ & $\mathrm{C} 8-\mathrm{H} 8$ & 0.9500 \\
\hline $\mathrm{Mo} 2-\mathrm{Cl} 5$ & $2.4138(6)$ & $\mathrm{C} 9-\mathrm{H} 9 \mathrm{~A}$ & 0.9800 \\
\hline $\mathrm{Mo} 3-\mathrm{Cl1}^{\mathrm{i}}$ & $2.4754(6)$ & $\mathrm{C} 9-\mathrm{H} 9 \mathrm{~B}$ & 0.9800 \\
\hline $\mathrm{Mo} 3-\mathrm{Cl} 2^{\mathrm{i}}$ & $2.4639(6)$ & $\mathrm{C} 9-\mathrm{H} 9 \mathrm{C}$ & 0.9800 \\
\hline $\mathrm{Mo} 3-\mathrm{Cl} 3$ & $2.4695(6)$ & $\mathrm{O} 2-\mathrm{C} 10$ & $1.239(3)$ \\
\hline $\mathrm{Mo} 3-\mathrm{Cl} 4$ & $2.4652(6)$ & $\mathrm{N} 3-\mathrm{C} 10$ & $1.299(3)$ \\
\hline $\mathrm{Mo} 3-\mathrm{Cl} 6$ & $2.4288(6)$ & $\mathrm{N} 3-\mathrm{C} 11$ & $1.461(3)$ \\
\hline $\mathrm{N} 1-\mathrm{H} 1 \mathrm{~A}$ & $0.89(3)$ & $\mathrm{N} 3-\mathrm{C} 12$ & $1.449(3)$ \\
\hline $\mathrm{N} 1-\mathrm{H} 1 \mathrm{~B}$ & $0.91(3)$ & $\mathrm{C} 10-\mathrm{H} 10$ & 0.9500 \\
\hline $\mathrm{N} 1-\mathrm{H} 1 \mathrm{C}$ & $0.91(3)$ & $\mathrm{C} 11-\mathrm{H} 11 \mathrm{~A}$ & 0.9800 \\
\hline $\mathrm{N} 1-\mathrm{C} 1$ & $1.465(3)$ & $\mathrm{C} 11-\mathrm{H} 11 \mathrm{~B}$ & 0.9800 \\
\hline $\mathrm{C} 1-\mathrm{C} 2$ & $1.376(3)$ & $\mathrm{C} 11-\mathrm{H} 11 \mathrm{C}$ & 0.9800 \\
\hline $\mathrm{C} 1-\mathrm{C} 5$ & $1.371(3)$ & $\mathrm{C} 12-\mathrm{H} 12 \mathrm{~A}$ & 0.9800 \\
\hline $\mathrm{C} 2-\mathrm{H} 2$ & 0.9500 & $\mathrm{C} 12-\mathrm{H} 12 \mathrm{~B}$ & 0.9800 \\
\hline $\mathrm{C} 2-\mathrm{C} 3$ & $1.378(4)$ & $\mathrm{C} 12-\mathrm{H} 12 \mathrm{C}$ & 0.9800 \\
\hline Mo2i-Mo1-Mo2 & $89.753(11)$ & $\mathrm{Cl} 3-\mathrm{Mo} 3-\mathrm{Mo}^{\mathrm{i}}$ & $118.261(15)$ \\
\hline Mo2-Mo1-Mo3 ${ }^{\mathrm{i}}$ & $60.013(9)$ & $\mathrm{Cl} 3-\mathrm{Mo} 3-\mathrm{Mo} 2^{\mathrm{i}}$ & $58.306(14)$ \\
\hline $\mathrm{Mo} 2^{\mathrm{i}}-\mathrm{Mo} 1-\mathrm{Mo}^{\mathrm{i}}$ & $59.675(10)$ & $\mathrm{Cl} 3-\mathrm{Mo} 3-\mathrm{Mo} 2$ & $118.588(17)$ \\
\hline $\mathrm{Mo3}-\mathrm{Mo1}-\mathrm{Mo} 2^{\mathrm{i}}$ & $60.080(8)$ & $\mathrm{Cl} 3-\mathrm{Mo} 3-\mathrm{Cl}^{\mathrm{i}}$ & $89.67(2)$ \\
\hline Mo3-Mo1-Mo2 & $59.698(9)$ & $\mathrm{Cl} 4-\mathrm{Mo} 3-\mathrm{Mol}^{\mathrm{i}}$ & $118.636(17)$ \\
\hline Mo3-Mo1-Mo3 ${ }^{\mathrm{i}}$ & $89.786(11)$ & $\mathrm{Cl} 4-\mathrm{Mo} 3-\mathrm{Mo} 1$ & $58.254(14)$ \\
\hline $\mathrm{Cl1}-\mathrm{Mo} 1-\mathrm{Mo} 2^{\mathrm{i}}$ & $118.020(15)$ & $\mathrm{Cl} 4-\mathrm{Mo} 3-\mathrm{Mo} 2$ & $58.556(14)$ \\
\hline $\mathrm{C} 11-\mathrm{Mo} 1-\mathrm{Mo} 2$ & $58.214(16)$ & $\mathrm{Cl} 4-\mathrm{Mo} 3-\mathrm{Mo} 2^{\mathrm{i}}$ & $118.205(16)$ \\
\hline $\mathrm{C} 11-\mathrm{Mo} 1-\mathrm{Mo} 3$ & $117.899(15)$ & $\mathrm{Cl} 4-\mathrm{Mo} 3-\mathrm{Cl1}^{\mathrm{i}}$ & $175.592(19)$ \\
\hline $\mathrm{C} 11-\mathrm{Mo} 1-\mathrm{Mo}^{\mathrm{i}}$ & $58.365(14)$ & $\mathrm{Cl} 4-\mathrm{Mo} 3-\mathrm{Cl} 3$ & $89.93(2)$ \\
\hline $\mathrm{Cl1}-\mathrm{Mo} 1-\mathrm{Cl} 2$ & $89.683(19)$ & $\mathrm{Cl} 6-\mathrm{Mo} 3-\mathrm{Mo} 1$ & $134.228(19)$ \\
\hline $\mathrm{Cl1}-\mathrm{Mo1}-\mathrm{Cl} 3$ & $175.251(19)$ & $\mathrm{Cl} 6-\mathrm{Mo} 3-\mathrm{Mol}^{\mathrm{i}}$ & $135.485(18)$ \\
\hline $\mathrm{Cl1}-\mathrm{Mol}-\mathrm{Cl} 4$ & $89.963(19)$ & $\mathrm{Cl} 6-\mathrm{Mo} 3-\mathrm{Mo}^{\mathrm{i}}$ & $137.228(18)$ \\
\hline $\mathrm{Cl} 2-\mathrm{Mo} 1-\mathrm{Mo} 2^{\mathrm{i}}$ & $58.387(15)$ & $\mathrm{Cl} 6-\mathrm{Mo} 3-\mathrm{Mo} 2$ & $132.798(17)$ \\
\hline $\mathrm{Cl} 2-\mathrm{Mo} 1-\mathrm{Mo} 2$ & $117.999(16)$ & $\mathrm{Cl} 6-\mathrm{Mo} 3-\mathrm{Cl1}^{\mathrm{i}}$ & $94.40(2)$ \\
\hline $\mathrm{Cl} 2-\mathrm{Mo} 1-\mathrm{Mo} 3$ & $118.444(17)$ & $\mathrm{Cl} 6-\mathrm{Mo} 3-\mathrm{Cl}^{\mathrm{i}}$ & $90.84(2)$ \\
\hline
\end{tabular}




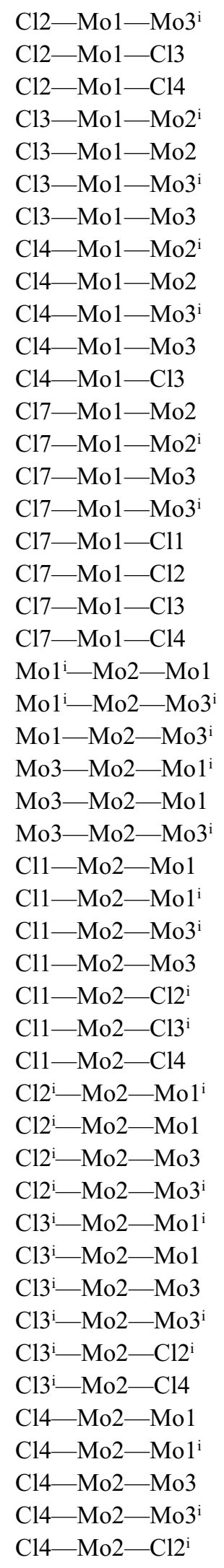

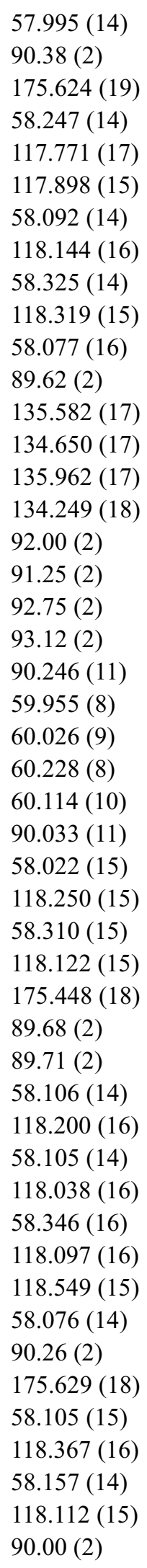

$\mathrm{Cl} 6-\mathrm{Mo} 3-\mathrm{Cl} 3$

$\mathrm{Cl} 6-\mathrm{Mo} 3-\mathrm{Cl} 4$

Mo1-Cl1-Mo2

$\mathrm{Mo} 1-\mathrm{Cl1}-\mathrm{Mo}^{\mathrm{i}}$

$\mathrm{Mo} 2-\mathrm{Cl1}-\mathrm{Mo}^{\mathrm{i}}$

$\mathrm{Mo} 1-\mathrm{Cl} 2-\mathrm{Mo}^{\mathrm{i}}$

$\mathrm{Mo} 3$ - $\mathrm{Cl} 2-\mathrm{Mo} 1$

$\mathrm{Mo}^{\mathrm{i}}-\mathrm{Cl} 2-\mathrm{Mo}^{\mathrm{i}}$

$\mathrm{Mo} 2-\mathrm{Cl} 3-\mathrm{Mo} 1$

$\mathrm{Mo} 3-\mathrm{Cl} 3-\mathrm{Mo} 1$

$\mathrm{Mo} 3-\mathrm{Cl} 3-\mathrm{Mo}^{\mathrm{i}}$

$\mathrm{Mo} 1-\mathrm{Cl} 4-\mathrm{Mo} 2$

Mo3-Cl4-Mo1

Mo3- $\mathrm{Cl} 4-\mathrm{Mo} 2$

H1A-N1-H1B

$\mathrm{H} 1 \mathrm{~A}-\mathrm{N} 1-\mathrm{H} 1 \mathrm{C}$

$\mathrm{H} 1 \mathrm{~B}-\mathrm{N} 1-\mathrm{H} 1 \mathrm{C}$

$\mathrm{C} 1-\mathrm{N} 1-\mathrm{H} 1 \mathrm{~A}$

C1-N1-H1B

$\mathrm{C} 1-\mathrm{N} 1-\mathrm{H} 1 \mathrm{C}$

$\mathrm{C} 2-\mathrm{C} 1-\mathrm{N} 1$

$\mathrm{C} 5-\mathrm{C} 1-\mathrm{N} 1$

$\mathrm{C} 5-\mathrm{C} 1-\mathrm{C} 2$

$\mathrm{C} 1-\mathrm{C} 2-\mathrm{H} 2$

$\mathrm{C} 1-\mathrm{C} 2-\mathrm{C} 3$

$\mathrm{C} 3-\mathrm{C} 2-\mathrm{H} 2$

$\mathrm{C} 2-\mathrm{C} 3-\mathrm{H} 3$

$\mathrm{C} 4-\mathrm{C} 3-\mathrm{C} 2$

$\mathrm{C} 4-\mathrm{C} 3-\mathrm{H} 3$

$\mathrm{C} 3-\mathrm{C} 4-\mathrm{H} 4$

$\mathrm{C} 3-\mathrm{C} 4-\mathrm{C} 6$

$\mathrm{C} 6-\mathrm{C} 4-\mathrm{H} 4$

$\mathrm{C} 1-\mathrm{C} 5-\mathrm{H} 5$

$\mathrm{C} 1-\mathrm{C} 5-\mathrm{C} 6$

C6- C5-H5

$\mathrm{C} 4-\mathrm{C} 6-\mathrm{C} 5$

$\mathrm{C} 4-\mathrm{C} 6-\mathrm{H} 6$

$\mathrm{C} 5-\mathrm{C} 6-\mathrm{H} 6$

$\mathrm{C} 7-\mathrm{N} 2-\mathrm{C} 9$

$\mathrm{C} 8-\mathrm{N} 2-\mathrm{C} 7$

$\mathrm{C} 8-\mathrm{N} 2-\mathrm{C} 9$

$\mathrm{N} 2-\mathrm{C} 7-\mathrm{H} 7 \mathrm{~A}$

N2-C7-H7B

$\mathrm{N} 2-\mathrm{C} 7-\mathrm{H} 7 \mathrm{C}$

$\mathrm{H} 7 \mathrm{~A}-\mathrm{C} 7-\mathrm{H} 7 \mathrm{~B}$

$\mathrm{H} 7 \mathrm{~A}-\mathrm{C} 7-\mathrm{H} 7 \mathrm{C}$

$\mathrm{H} 7 \mathrm{~B}-\mathrm{C} 7-\mathrm{H} 7 \mathrm{C}$

$\mathrm{O} 1-\mathrm{C} 8-\mathrm{N} 2$
$93.06(2)$

$90.01(2)$

$63.764(15)$

$63.713(15)$

$63.630(15)$

$63.507(16)$

$63.792(15)$

$63.287(16)$

$63.408(14)$

$63.489(16)$

$63.616(15)$

$63.569(15)$

$63.669(15)$

$63.286(16)$

101 (2)

114 (3)

109 (2)

108.4 (18)

$113.6(18)$

111.0 (18)

119.1 (2)

$119.1(2)$

121.8 (2)

120.6

118.8 (3)

120.6

119.7

120.5 (3)

119.7

120.0

120.0 (3)

120.0

120.7

$118.6(3)$

120.7

120.2 (3)

119.9

119.9

117.3 (2)

$121.2(2)$

121.5 (2)

109.5

109.5

109.5

109.5

109.5

109.5

124.7 (2) 


$$
\begin{aligned}
& \mathrm{Cl} 5-\mathrm{Mo} 2-\mathrm{Mo}^{\mathrm{i}} \\
& \mathrm{Cl} 5-\mathrm{Mo} 2-\mathrm{Mo} 1 \\
& \mathrm{Cl} 5-\mathrm{Mo} 2-\mathrm{Mo}^{\mathrm{i}} \\
& \text { Cl5-Mo2-Mo3 } \\
& \mathrm{Cl} 5-\mathrm{Mo} 2-\mathrm{Cl} 1 \\
& \mathrm{Cl} 5-\mathrm{Mo} 2-\mathrm{Cl}_{2}^{\mathrm{i}} \\
& \mathrm{Cl} 5-\mathrm{Mo} 2-\mathrm{Cl}^{\mathrm{i}} \\
& \mathrm{Cl} 5-\mathrm{Mo} 2-\mathrm{Cl} 4 \\
& \text { Mo1-Mo3-Mo1 }{ }^{\mathrm{i}} \\
& \mathrm{Mo1}-\mathrm{Mo3}-\mathrm{Mo}^{\mathrm{i}} \\
& \mathrm{Mo} 2-\mathrm{Mo3}-\mathrm{Mol}^{\mathrm{i}} \\
& \text { Mo2 }-\mathrm{Mo3}-\mathrm{Mol}^{\mathrm{i}} \\
& \text { Mo2-Mo3-Mo1 } \\
& \text { Mo2-Mo3-Mo2 }{ }^{\mathrm{i}} \\
& \mathrm{Cl1} \text { - } \mathrm{Mo} 3-\mathrm{Mol}^{\mathrm{i}} \\
& \text { Cl1 }- \text { Mo3-Mo1 } \\
& \text { Cl1 }-\mathrm{Mo} 3-\mathrm{Mo} 2 \\
& \mathrm{Cl} 1{ }^{\mathrm{i}}-\mathrm{Mo} 3-\mathrm{Mo}^{2} \\
& \mathrm{Cl} 2^{\mathrm{i}}-\mathrm{Mo} 3-\mathrm{Mo}^{\mathrm{i}} \\
& \mathrm{Cl} 2 \text { - }-\mathrm{Mo} 3-\mathrm{Mo} 1 \\
& \mathrm{Cl} 2 \text { - }-\mathrm{Mo} 3-\mathrm{Mo} 2 \\
& \mathrm{C} 12^{\mathrm{i}}-\mathrm{Mo} 3-\mathrm{Mo}^{\mathrm{i}} \\
& \mathrm{Cl} 2-\mathrm{Mo} 3-\mathrm{Cl}^{\mathrm{i}} \\
& \mathrm{Cl} 2-\mathrm{Mo} 3-\mathrm{Cl} 3 \\
& \mathrm{Cl} 2-\mathrm{Mo} 3-\mathrm{Cl} 4 \\
& \mathrm{Cl} 3-\mathrm{Mo} 3-\mathrm{Mo} 1 \\
& \mathrm{~N} 1-\mathrm{C} 1-\mathrm{C} 2-\mathrm{C} 3 \\
& \mathrm{~N} 1-\mathrm{C} 1-\mathrm{C} 5-\mathrm{C} 6 \\
& \mathrm{C} 1-\mathrm{C} 2-\mathrm{C} 3-\mathrm{C} 4 \\
& \mathrm{C} 1-\mathrm{C} 5-\mathrm{C} 6-\mathrm{C} 4 \\
& \mathrm{C} 2-\mathrm{C} 1-\mathrm{C} 5-\mathrm{C} 6 \\
& \mathrm{C} 2-\mathrm{C} 3-\mathrm{C} 4-\mathrm{C} 6
\end{aligned}
$$

\begin{tabular}{|c|c|}
\hline $\mathrm{O} 1-\mathrm{C} 8-\mathrm{H} 8$ & 117.6 \\
\hline $\mathrm{N} 2-\mathrm{C} 8-\mathrm{H} 8$ & 117.6 \\
\hline $\mathrm{N} 2-\mathrm{C} 9-\mathrm{H} 9 \mathrm{~A}$ & 109.5 \\
\hline $\mathrm{N} 2-\mathrm{C} 9-\mathrm{H} 9 \mathrm{~B}$ & 109.5 \\
\hline $\mathrm{N} 2-\mathrm{C} 9-\mathrm{H} 9 \mathrm{C}$ & 109.5 \\
\hline $\mathrm{H} 9 \mathrm{~A}-\mathrm{C} 9-\mathrm{H} 9 \mathrm{~B}$ & 109.5 \\
\hline $\mathrm{H} 9 \mathrm{~A}-\mathrm{C} 9-\mathrm{H} 9 \mathrm{C}$ & 109.5 \\
\hline $\mathrm{H} 9 \mathrm{~B}-\mathrm{C} 9-\mathrm{H} 9 \mathrm{C}$ & 109.5 \\
\hline $\mathrm{C} 10-\mathrm{N} 3-\mathrm{C} 11$ & $122.7(2)$ \\
\hline $\mathrm{C} 10-\mathrm{N} 3-\mathrm{C} 12$ & $121.0(2)$ \\
\hline $\mathrm{C} 12-\mathrm{N} 3-\mathrm{C} 11$ & $116.3(2)$ \\
\hline $\mathrm{O} 2-\mathrm{C} 10-\mathrm{N} 3$ & $126.1(3)$ \\
\hline $\mathrm{O} 2-\mathrm{C} 10-\mathrm{H} 10$ & 116.9 \\
\hline $\mathrm{N} 3-\mathrm{C} 10-\mathrm{H} 10$ & 116.9 \\
\hline $\mathrm{N} 3-\mathrm{C} 11-\mathrm{H} 11 \mathrm{~A}$ & 109.5 \\
\hline $\mathrm{N} 3-\mathrm{C} 11-\mathrm{H} 11 \mathrm{~B}$ & 109.5 \\
\hline $\mathrm{N} 3-\mathrm{C} 11-\mathrm{H} 11 \mathrm{C}$ & 109.5 \\
\hline $\mathrm{H} 11 \mathrm{~A}-\mathrm{C} 11-\mathrm{H} 11 \mathrm{~B}$ & 109.5 \\
\hline $\mathrm{H} 11 \mathrm{~A}-\mathrm{C} 11-\mathrm{H} 11 \mathrm{C}$ & 109.5 \\
\hline $\mathrm{H} 11 \mathrm{~B}-\mathrm{C} 11-\mathrm{H} 11 \mathrm{C}$ & 109.5 \\
\hline $\mathrm{N} 3-\mathrm{C} 12-\mathrm{H} 12 \mathrm{~A}$ & 109.5 \\
\hline $\mathrm{N} 3-\mathrm{C} 12-\mathrm{H} 12 \mathrm{~B}$ & 109.5 \\
\hline $\mathrm{N} 3-\mathrm{C} 12-\mathrm{H} 12 \mathrm{C}$ & 109.5 \\
\hline $\mathrm{H} 12 \mathrm{~A}-\mathrm{C} 12-\mathrm{H} 12 \mathrm{~B}$ & 109.5 \\
\hline $\mathrm{H} 12 \mathrm{~A}-\mathrm{C} 12-\mathrm{H} 12 \mathrm{C}$ & 109.5 \\
\hline $\mathrm{H} 12 \mathrm{~B}-\mathrm{C} 12-\mathrm{H} 12 \mathrm{C}$ & 109.5 \\
\hline $\mathrm{C} 3-\mathrm{C} 4-\mathrm{C} 6-\mathrm{C} 5$ & $0.4(4)$ \\
\hline $\mathrm{C} 5-\mathrm{C} 1-\mathrm{C} 2-\mathrm{C} 3$ & $-0.5(4)$ \\
\hline $\mathrm{C} 7-\mathrm{N} 2-\mathrm{C} 8-\mathrm{O} 1$ & $0.0(4)$ \\
\hline $\mathrm{C} 9-\mathrm{N} 2-\mathrm{C} 8-\mathrm{O} 1$ & $-179.2(2$ \\
\hline $\mathrm{C} 11-\mathrm{N} 3-\mathrm{C} 10-\mathrm{O} 2$ & -177.1 \\
\hline $\mathrm{C} 12-\mathrm{N} 3-\mathrm{C} 10-\mathrm{O} 2$ & $1.5(5)$ \\
\hline
\end{tabular}

$134.531(18)$
$135.222(19)$
$135.276(17)$
$134.689(19)$
$92.63(2)$
$91.92(2)$
$92.04(2)$
$92.31(2)$
$90.214(10)$
$59.965(10)$
$60.098(8)$
$59.961(8)$
$60.188(8)$
$89.968(10)$
$57.923(15)$
$118.008(15)$
$118.001(15)$
$58.058(14)$
$58.214(14)$
$118.777(16)$
$58.608(16)$
$118.165(15)$
$89.55(2)$
$176.071(19)$
$90.56(2)$
$58.419(15)$
$179.4(2)$
$-178.9(2)$
$0.0(4)$
$-0.8(4)$
$0.9(4)$
$0.1(4)$

Symmetry code: (i) $-x+1,-y+1,-z+1$.

Hydrogen-bond geometry $\left(\AA,{ }^{\circ}\right)$

\begin{tabular}{lllll}
\hline$D-\mathrm{H} \cdots A$ & $D-\mathrm{H}$ & $\mathrm{H} \cdots A$ & $D \cdots A$ & $D-\mathrm{H} \cdots A$ \\
\hline $\mathrm{N} 1-\mathrm{H} 1 A \cdots \mathrm{O} 2^{\mathrm{ii}}$ & $0.89(3)$ & $2.01(3)$ & $2.827(3)$ & $152(2)$ \\
$\mathrm{N} 1-\mathrm{H} 1 B \cdots \mathrm{O} 2^{\mathrm{iii}}$ & $0.91(3)$ & $1.94(3)$ & $2.833(3)$ & $168(3)$ \\
$\mathrm{N} 1-\mathrm{H} 1 C \cdots \mathrm{O} 1^{\text {iv }}$ & $0.91(3)$ & $1.82(3)$ & $2.715(3)$ & $166(3)$ \\
\hline
\end{tabular}

Symmetry codes: (ii) $-x+1,-y+1,-z$; (iii) $x-1, y, z$; (iv) $x, y, z-1$. 
p-Phenylenediammonium octa- $\mu_{3}$-chlorido-hexachlorido-octahedro-hexamolybdate $N, N$-dimethylformamide hexasolvate (2)

\section{Crystal data}

$\left(\mathrm{C}_{6} \mathrm{H}_{10} \mathrm{~N}_{2}\right)\left[\mathrm{Mo}_{6} \mathrm{Cl}_{8} \mathrm{Cl}_{6}\right] \cdot 6 \mathrm{C}_{3} \mathrm{H}_{7} \mathrm{NO}$

$M_{r}=1620.67$

Triclinic, $P \overline{1}$

$a=10.1752(16) \AA$

$b=10.3227(16) \AA$

$c=13.736(2) \AA$

$\alpha=95.204(4)^{\circ}$

$\beta=111.483(4)^{\circ}$

$\gamma=101.973(4)^{\circ}$

$V=1291.1(3) \AA^{3}$

\section{Data collection}

Bruker SMART X2S benchtop diffractometer

Radiation source: sealed microfocus source, XOS X-beam microfocus source

Graphite monochromator

Detector resolution: 8.3330 pixels $\mathrm{mm}^{-1}$

$\varphi$ and $\omega$ scans

Absorption correction: multi-scan

(SADABS; Bruker, 2012)

\section{Refinement}

Refinement on $F^{2}$

Least-squares matrix: full

$R\left[F^{2}>2 \sigma\left(F^{2}\right)\right]=0.026$

$w R\left(F^{2}\right)=0.062$

$S=1.03$

4504 reflections

285 parameters

144 restraints

Primary atom site location: structure-invariant direct methods

Hydrogen site location: mixed
$Z=1$

$F(000)=790$

$D_{\mathrm{x}}=2.084 \mathrm{Mg} \mathrm{m}^{-3}$

Mo $K \alpha$ radiation, $\lambda=0.71073 \AA$

Cell parameters from 4690 reflections

$\theta=2.2-25.0^{\circ}$

$\mu=2.18 \mathrm{~mm}^{-1}$

$T=200 \mathrm{~K}$

Needle, yellow

$0.50 \times 0.13 \times 0.13 \mathrm{~mm}$

$T_{\min }=0.552, T_{\max }=0.745$

12459 measured reflections

4504 independent reflections

3666 reflections with $I>2 \sigma(I)$

$R_{\text {int }}=0.035$

$\theta_{\text {max }}=25.0^{\circ}, \theta_{\min }=2.4^{\circ}$

$h=-12 \rightarrow 12$

$k=-12 \rightarrow 12$

$l=-16 \rightarrow 16$

$\mathrm{H}$ atoms treated by a mixture of independent and constrained refinement

$w=1 /\left[\sigma^{2}\left(F_{\mathrm{o}}^{2}\right)+(0.0182 P)^{2}+0.7035 P\right]$ where $P=\left(F_{\mathrm{o}}^{2}+2 F_{\mathrm{c}}^{2}\right) / 3$

$(\Delta / \sigma)_{\max }=0.002$

$\Delta \rho_{\max }=0.66 \mathrm{e} \AA^{-3}$

$\Delta \rho_{\min }=-0.56$ e $\AA^{-3}$

Extinction correction: SHELXL2018

(Sheldrick, 2015),

$\mathrm{Fc}^{*}=\mathrm{kFc}\left[1+0.001 \mathrm{xFc}^{2} \lambda^{3} / \sin (2 \theta)\right]^{-1 / 4}$

Extinction coefficient: 0.0019 (2)

\section{Special details}

Geometry. All esds (except the esd in the dihedral angle between two 1.s. planes) are estimated using the full covariance matrix. The cell esds are taken into account individually in the estimation of esds in distances, angles and torsion angles; correlations between esds in cell parameters are only used when they are defined by crystal symmetry. An approximate (isotropic) treatment of cell esds is used for estimating esds involving l.s. planes.

Fractional atomic coordinates and isotropic or equivalent isotropic displacement parameters $\left(\AA^{2}\right)$

\begin{tabular}{llllll}
\hline & $x$ & $y$ & $z$ & $U_{\text {iss }} / U_{\text {eq }}$ & Occ. $(<1)$ \\
\hline Mo1 & $0.38576(3)$ & $0.32569(3)$ & $0.46504(2)$ & $0.01984(9)$ & \\
Mo2 & $0.38226(3)$ & $0.53457(3)$ & $0.37203(2)$ & $0.01942(9)$ & \\
Mo3 & $0.61719(3)$ & $0.44772(3)$ & $0.43759(2)$ & $0.01961(9)$ & \\
C11 & $0.39098(9)$ & $0.31721(8)$ & $0.28606(6)$ & $0.02470(19)$ & \\
C12 & $0.16889(8)$ & $0.41769(8)$ & $0.40411(7)$ & $0.02485(19)$ &
\end{tabular}




\begin{tabular}{|c|c|c|c|c|c|}
\hline $\mathrm{Cl} 3$ & $0.61432(9)$ & $0.25211(8)$ & $0.52889(7)$ & $0.02446(19)$ & \\
\hline $\mathrm{Cl} 4$ & $0.39296(9)$ & $0.35261(8)$ & $0.64765(6)$ & $0.02436(19)$ & \\
\hline $\mathrm{Cl} 5$ & $0.23637(10)$ & $0.09538(8)$ & $0.42076(7)$ & $0.0329(2)$ & \\
\hline $\mathrm{Cl} 6$ & $0.22927(9)$ & $0.58540(9)$ & $0.20362(7)$ & $0.0303(2)$ & \\
\hline $\mathrm{Cl} 7$ & $0.77350(10)$ & $0.38668(9)$ & $0.35511(7)$ & $0.0340(2)$ & \\
\hline N1 & $0.6572(4)$ & 0.7709 (3) & $0.0311(3)$ & $0.0349(8)$ & \\
\hline H1A & $0.593(4)$ & $0.816(4)$ & $-0.008(3)$ & $0.052 *$ & \\
\hline H1B & $0.735(3)$ & $0.773(4)$ & $0.010(3)$ & $0.052 *$ & \\
\hline $\mathrm{H} 1 \mathrm{C}$ & $0.702(4)$ & $0.814(4)$ & $0.1013(17)$ & $0.052 *$ & \\
\hline $\mathrm{C} 1$ & $0.5771(4)$ & $0.6310(4)$ & $0.0161(3)$ & $0.0300(8)$ & \\
\hline $\mathrm{C} 2$ & $0.4838(4)$ & $0.5982(4)$ & $0.0660(3)$ & $0.0346(10)$ & $0.918(4)$ \\
\hline $\mathrm{H} 2$ & 0.473071 & 0.666286 & 0.111672 & $0.041^{*}$ & $0.918(4)$ \\
\hline $\mathrm{C} 2 \mathrm{~A}$ & $0.614(4)$ & $0.562(3)$ & $0.098(2)$ & $0.0346(10)$ & $0.082(4)$ \\
\hline $\mathrm{H} 2 \mathrm{~A}$ & 0.690135 & 0.603414 & 0.164923 & $0.041 *$ & $0.082(4)$ \\
\hline $\mathrm{C} 3$ & $0.5946(4)$ & $0.5334(4)$ & $-0.0500(3)$ & $0.0368(11)$ & 0.918 (4) \\
\hline H3 & 0.659871 & 0.556614 & -0.084221 & $0.044 *$ & $0.918(4)$ \\
\hline C3A & $0.465(2)$ & $0.570(3)$ & $-0.0792(18)$ & $0.0368(11)$ & $0.082(4)$ \\
\hline $\mathrm{H} 3 \mathrm{~A}$ & 0.440687 & 0.620672 & -0.134626 & $0.044 *$ & $0.082(4)$ \\
\hline $\mathrm{O} 1$ & $0.2262(3)$ & $0.1030(3)$ & $0.7614(2)$ & $0.0397(7)$ & \\
\hline N2 & 0.0590 & $0.1987(3)$ & $0.6536(2)$ & $0.0345(7)$ & \\
\hline $\mathrm{C} 4$ & $-0.0193(4)$ & $0.2197(4)$ & $0.5464(3)$ & $0.0471(11)$ & \\
\hline H4A & 0.012128 & 0.173444 & 0.496723 & $0.071^{*}$ & \\
\hline H4B & 0.001881 & 0.316351 & 0.544827 & $0.071^{*}$ & \\
\hline $\mathrm{H} 4 \mathrm{C}$ & -0.124667 & 0.183480 & 0.525552 & $0.071^{*}$ & \\
\hline C5 & $0.0344(6)$ & $0.2671(6)$ & $0.7385(4)$ & $0.089(2)$ & \\
\hline H5A & 0.105377 & 0.355397 & 0.767114 & $0.133^{*}$ & \\
\hline H5B & 0.045962 & 0.213536 & 0.795020 & $0.133^{*}$ & \\
\hline $\mathrm{H} 5 \mathrm{C}$ & -0.064912 & 0.279168 & 0.711359 & $0.133^{*}$ & \\
\hline C6 & $0.1511(4)$ & $0.1229(3)$ & $0.6738(3)$ & $0.0310(9)$ & \\
\hline H6 & 0.160825 & 0.078393 & 0.614092 & $0.037^{*}$ & \\
\hline $\mathrm{O} 2$ & $0.4557(3)$ & -0.1235 & $0.9024(2)$ & $0.0528(8)$ & \\
\hline N3 & $0.5360(3)$ & $-0.0093(3)$ & $0.7928(2)$ & $0.0365(8)$ & \\
\hline $\mathrm{C} 7$ & $0.6841(5)$ & $0.0426(5)$ & $0.8704(4)$ & $0.0737(16)$ & \\
\hline H7A & 0.742668 & -0.019070 & 0.863107 & $0.111^{*}$ & \\
\hline H7B & 0.684310 & 0.050996 & 0.942109 & $0.111^{*}$ & \\
\hline $\mathrm{H} 7 \mathrm{C}$ & 0.726072 & 0.131344 & 0.858773 & $0.111^{*}$ & \\
\hline $\mathrm{C} 8$ & $0.5004(5)$ & $0.0294(4)$ & $0.6892(3)$ & $0.0521(12)$ & \\
\hline H8A & 0.516051 & 0.127409 & 0.697400 & $0.078^{*}$ & \\
\hline H8B & 0.397800 & -0.014548 & 0.643792 & $0.078^{*}$ & \\
\hline $\mathrm{H} 8 \mathrm{C}$ & 0.563372 & 0.001622 & 0.656359 & $0.078^{*}$ & \\
\hline C9 & $0.4359(4)$ & -0.0854 (4) & $0.8172(4)$ & $0.0401(10)$ & \\
\hline H9 & 0.339326 & -0.113538 & 0.763903 & $0.048 *$ & \\
\hline $\mathrm{O} 3$ & $1.1120(3)$ & $0.2000(3)$ & $0.0265(2)$ & $0.0531(8)$ & \\
\hline N4 & $0.9538(3)$ & $0.2480(3)$ & $0.0950(2)$ & $0.0348(7)$ & \\
\hline C10 & $0.8672(5)$ & $0.3384(4)$ & 0.1084 (4) & $0.0542(12)$ & \\
\hline H10A & 0.910401 & 0.386797 & 0.182173 & $0.081 *$ & \\
\hline H10B & 0.767006 & 0.286161 & 0.091710 & $0.081^{*}$ & \\
\hline $\mathrm{H} 10 \mathrm{C}$ & 0.865666 & 0.403223 & 0.060362 & $0.081 *$ & \\
\hline
\end{tabular}




$\begin{array}{lllll}\text { C11 } & 0.9567(5) & 0.1356(4) & 0.1512(3) & 0.0513(11) \\ \text { H11A } & 0.986243 & 0.066079 & 0.116745 & 0.077^{*} \\ \text { H11B } & 0.859138 & 0.097880 & 0.149607 & 0.077^{*} \\ \text { H11C } & 1.026857 & 0.166773 & 0.225219 & 0.077^{*} \\ \text { C12 } & 1.0327(4) & 0.2695(4) & 0.0379(3) & 0.0415(10) \\ \text { H12 } & 1.027845 & 0.344630 & 0.002820 & 0.050^{*}\end{array}$

Atomic displacement parameters $\left(\AA^{2}\right)$

\begin{tabular}{|c|c|c|c|c|c|c|}
\hline & $U^{11}$ & $U^{22}$ & $U^{33}$ & $U^{12}$ & $U^{13}$ & $U^{23}$ \\
\hline Mo1 & $0.01844(16)$ & $0.01776(16)$ & $0.02281(18)$ & $0.00192(12)$ & $0.00933(13)$ & $0.00253(12)$ \\
\hline Mo2 & $0.01778(16)$ & $0.01982(16)$ & $0.02082(18)$ & $0.00380(12)$ & $0.00849(13)$ & $0.00326(12)$ \\
\hline Mo3 & $0.01810(16)$ & $0.01988(16)$ & $0.02220(18)$ & $0.00434(12)$ & $0.01006(13)$ & $0.00274(12)$ \\
\hline $\mathrm{Cl1}$ & $0.0253(4)$ & $0.0231(4)$ & $0.0234(5)$ & $0.0034(3)$ & $0.0097(4)$ & $-0.0003(3)$ \\
\hline $\mathrm{C} 12$ & $0.0174(4)$ & $0.0274(4)$ & $0.0279(5)$ & $0.0026(3)$ & $0.0088(4)$ & $0.0041(4)$ \\
\hline $\mathrm{Cl} 3$ & $0.0252(4)$ & $0.0212(4)$ & $0.0287(5)$ & $0.0079(3)$ & $0.0115(4)$ & $0.0047(4)$ \\
\hline $\mathrm{Cl} 4$ & 0.0249 (4) & $0.0248(4)$ & $0.0264(5)$ & $0.0041(3)$ & $0.0142(4)$ & $0.0067(4)$ \\
\hline $\mathrm{Cl} 5$ & $0.0336(5)$ & $0.0222(4)$ & $0.0385(5)$ & $-0.0019(4)$ & $0.0151(4)$ & 0.0018 \\
\hline $\mathrm{Cl} 6$ & $0.0290(5)$ & $0.0361(5)$ & $0.0254(5)$ & $0.0108(4)$ & $0.0087(4)$ & $0.0072(4)$ \\
\hline $\mathrm{Cl} 7$ & $0.0331(5)$ & $0.0407(5)$ & $0.0373(5)$ & $0.0137(4)$ & $0.0223(4)$ & $0.0051(4)$ \\
\hline N1 & $0.0345(19)$ & $0.039(2)$ & $0.0338(19)$ & $0.0056(16)$ & $0.0181(16)$ & $0.0081(16)$ \\
\hline $\mathrm{C} 1$ & $0.028(2)$ & $0.036(2)$ & $0.029(2)$ & $0.0097(17)$ & $0.0132(17)$ & $0.0136(17)$ \\
\hline $\mathrm{C} 2$ & $0.039(2)$ & $0.038(2)$ & $0.032(2)$ & $0.015(2)$ & $0.018(2)$ & $0.0055(19)$ \\
\hline $\mathrm{C} 2 \mathrm{~A}$ & $0.039(2)$ & $0.038(2)$ & $0.032(2)$ & $0.015(2)$ & $0.018(2)$ & $0.0055(19)$ \\
\hline C3 & $0.035(2)$ & $0.049(3)$ & $0.033(2)$ & $0.010(2)$ & $0.021(2)$ & $0.012(2)$ \\
\hline $\mathrm{C} 3 \mathrm{~A}$ & $0.035(2)$ & $0.049(3)$ & $0.033(2)$ & $0.010(2)$ & $0.021(2)$ & $0.012(2)$ \\
\hline $\mathrm{O} 1$ & $0.0426(16)$ & $0.0405(16)$ & $0.0385(17)$ & $0.0171(13)$ & $0.0145(14)$ & $0.0111(13)$ \\
\hline N2 & $0.0296(17)$ & $0.0395(19)$ & $0.040(2)$ & $0.0109(15)$ & $0.0191(15)$ & $0.0086(15)$ \\
\hline $\mathrm{C} 4$ & $0.030(2)$ & $0.060(3)$ & $0.054(3)$ & $0.013(2)$ & $0.015(2)$ & $0.027(2)$ \\
\hline $\mathrm{C} 5$ & $0.109(5)$ & $0.126(5)$ & $0.065(4)$ & $0.083(4)$ & $0.045(3)$ & $0.016(4)$ \\
\hline C6 & $0.032(2)$ & $0.0247(19)$ & $0.039(2)$ & $0.0027(17)$ & $0.0197(19)$ & $0.0024(17)$ \\
\hline $\mathrm{O} 2$ & $0.0527(19)$ & $0.0526(19)$ & $0.052(2)$ & $0.0122(15)$ & $0.0172(16)$ & $0.0249(16)$ \\
\hline N3 & $0.0329(18)$ & $0.0305(18)$ & $0.040(2)$ & $0.0080(15)$ & $0.0073(15)$ & $0.0089(15)$ \\
\hline $\mathrm{C} 7$ & $0.048(3)$ & $0.060(3)$ & $0.082(4)$ & $-0.008(3)$ & $-0.001(3)$ & $0.027(3)$ \\
\hline $\mathrm{C} 8$ & $0.076(3)$ & $0.054(3)$ & $0.041(3)$ & $0.032(3)$ & $0.029(2)$ & $0.016(2)$ \\
\hline C9 & $0.033(2)$ & $0.027(2)$ & $0.051(3)$ & $0.0096(18)$ & $0.007(2)$ & $0.003(2)$ \\
\hline $\mathrm{O} 3$ & $0.0490(18)$ & $0.0476(18)$ & $0.071(2)$ & $0.0059(15)$ & $0.0388(17)$ & $0.0033(16)$ \\
\hline N4 & $0.0308(17)$ & $0.0370(19)$ & $0.0326(19)$ & $0.0017(15)$ & $0.0122(15)$ & $0.0045(15)$ \\
\hline $\mathrm{C} 10$ & $0.047(3)$ & $0.057(3)$ & $0.064(3)$ & $0.012(2)$ & $0.028(2)$ & $0.008(2)$ \\
\hline C11 & $0.051(3)$ & $0.055(3)$ & 0.048 & $0.009(2)$ & $0.021(2)$ & $0.020(2)$ \\
\hline $\mathrm{C} 12$ & $0.039(2)$ & $0.040(2)$ & $0.037(2)$ & $-0.004(2)$ & $0.014(2)$ & $0.0043(19)$ \\
\hline
\end{tabular}

Geometric parameters $\left(A,{ }^{\circ}\right)$

\begin{tabular}{llll}
\hline Mo1-Mo2 & \\
Mo1-Mo2 & $2.6065(5)$ & C3A-H3A & 0.9500 \\
Mo1-Mo3 & $2.6040(5)$ & O1-C6 & $1.229(4)$ \\
Mo1-Mo3 & $2.6039(5)$ & N2-C4 & $1.456(5)$ \\
& $2.5984(5)$ & N2-C5 & $1.435(5)$
\end{tabular}




\begin{tabular}{|c|c|c|c|}
\hline Mo1-Cl1 & $2.4724(9)$ & $\mathrm{N} 2-\mathrm{C} 6$ & $1.312(4)$ \\
\hline $\mathrm{Mo} 1-\mathrm{Cl} 2$ & $2.4764(9)$ & $\mathrm{C} 4-\mathrm{H} 4 \mathrm{~A}$ & 0.9800 \\
\hline $\mathrm{Mo} 1-\mathrm{Cl} 3$ & $2.4727(9)$ & $\mathrm{C} 4-\mathrm{H} 4 \mathrm{~B}$ & 0.9800 \\
\hline $\mathrm{Mo} 1-\mathrm{Cl} 4$ & $2.4708(9)$ & $\mathrm{C} 4-\mathrm{H} 4 \mathrm{C}$ & 0.9800 \\
\hline Mo1-Cl5 & $2.4277(9)$ & $\mathrm{C} 5-\mathrm{H} 5 \mathrm{~A}$ & 0.9800 \\
\hline Mo2-Mo3 ${ }^{\mathrm{i}}$ & $2.6027(5)$ & $\mathrm{C} 5-\mathrm{H} 5 \mathrm{~B}$ & 0.9800 \\
\hline Mo2-Mo3 & $2.6055(5)$ & $\mathrm{C} 5-\mathrm{H} 5 \mathrm{C}$ & 0.9800 \\
\hline Mo2-Cl1 & $2.4729(9)$ & $\mathrm{C} 6-\mathrm{H} 6$ & 0.9500 \\
\hline $\mathrm{Mo} 2-\mathrm{Cl} 2$ & $2.4644(9)$ & $\mathrm{O} 2-\mathrm{C} 9$ & $1.228(5)$ \\
\hline $\mathrm{Mo} 2-\mathrm{Cl}^{\mathrm{i}}$ & $2.4682(9)$ & $\mathrm{N} 3-\mathrm{C} 7$ & $1.442(5)$ \\
\hline $\mathrm{Mo} 2-\mathrm{Cl}^{\mathrm{i}}$ & $2.4629(9)$ & $\mathrm{N} 3-\mathrm{C} 8$ & $1.450(5)$ \\
\hline $\mathrm{Mo} 2-\mathrm{Cl} 6$ & $2.4436(9)$ & N3-C9 & $1.316(5)$ \\
\hline $\mathrm{Mo} 3-\mathrm{Cl1}$ & $2.4781(9)$ & C7-H7A & 0.9800 \\
\hline $\mathrm{Mo} 3-\mathrm{Cl} 2^{\mathrm{i}}$ & $2.4751(9)$ & $\mathrm{C} 7-\mathrm{H} 7 \mathrm{~B}$ & 0.9800 \\
\hline $\mathrm{Mo} 3-\mathrm{Cl} 3$ & $2.4724(9)$ & $\mathrm{C} 7-\mathrm{H} 7 \mathrm{C}$ & 0.9800 \\
\hline $\mathrm{Mo} 3-\mathrm{Cl}^{\mathrm{i}}$ & $2.4632(9)$ & $\mathrm{C} 8-\mathrm{H} 8 \mathrm{~A}$ & 0.9800 \\
\hline $\mathrm{Mo} 3-\mathrm{Cl} 7$ & $2.4116(9)$ & $\mathrm{C} 8-\mathrm{H} 8 \mathrm{~B}$ & 0.9800 \\
\hline $\mathrm{N} 1-\mathrm{H} 1 \mathrm{~A}$ & $0.922(18)$ & $\mathrm{C} 8-\mathrm{H} 8 \mathrm{C}$ & 0.9800 \\
\hline $\mathrm{N} 1-\mathrm{H} 1 \mathrm{~B}$ & $0.927(18)$ & $\mathrm{C} 9-\mathrm{H} 9$ & 0.9500 \\
\hline $\mathrm{N} 1-\mathrm{H} 1 \mathrm{C}$ & $0.924(18)$ & $\mathrm{O} 3-\mathrm{C} 12$ & $1.227(5)$ \\
\hline $\mathrm{N} 1-\mathrm{C} 1$ & $1.458(5)$ & $\mathrm{N} 4-\mathrm{C} 10$ & $1.453(5)$ \\
\hline $\mathrm{C} 1-\mathrm{C} 2$ & $1.367(5)$ & $\mathrm{N} 4-\mathrm{C} 11$ & $1.450(5)$ \\
\hline $\mathrm{C} 1-\mathrm{C} 2 \mathrm{~A}$ & $1.361(16)$ & $\mathrm{N} 4-\mathrm{C} 12$ & $1.314(5)$ \\
\hline $\mathrm{C} 1-\mathrm{C} 3$ & $1.377(5)$ & $\mathrm{C} 10-\mathrm{H} 10 \mathrm{~A}$ & 0.9800 \\
\hline $\mathrm{C} 1-\mathrm{C} 3 \mathrm{~A}$ & $1.366(16)$ & $\mathrm{C} 10-\mathrm{H} 10 \mathrm{~B}$ & 0.9800 \\
\hline $\mathrm{C} 2-\mathrm{H} 2$ & 0.9500 & $\mathrm{C} 10-\mathrm{H} 10 \mathrm{C}$ & 0.9800 \\
\hline $\mathrm{C} 2-\mathrm{C} 3^{\mathrm{ii}}$ & $1.378(5)$ & $\mathrm{C} 11-\mathrm{H} 11 \mathrm{~A}$ & 0.9800 \\
\hline $\mathrm{C} 2 \mathrm{~A}-\mathrm{H} 2 \mathrm{~A}$ & 0.9500 & $\mathrm{C} 11-\mathrm{H} 11 \mathrm{~B}$ & 0.9800 \\
\hline $\mathrm{C} 2 \mathrm{~A}-\mathrm{C} 3 \mathrm{~A}^{\mathrm{ii}}$ & $1.380(15)$ & $\mathrm{C} 11-\mathrm{H} 11 \mathrm{C}$ & 0.9800 \\
\hline $\mathrm{C} 3-\mathrm{H} 3$ & 0.9500 & $\mathrm{C} 12-\mathrm{H} 12$ & 0.9500 \\
\hline $\mathrm{Mo} 2-\mathrm{Mo1}-\mathrm{Mo}^{\mathrm{i}}$ & $90.126(16)$ & $\mathrm{Cl} 4-\mathrm{Mo} 3-\mathrm{Cl} 3$ & $175.60(3)$ \\
\hline $\mathrm{Mo3}-\mathrm{Mo1}-\mathrm{Mo}^{\mathrm{i}}$ & $59.936(14)$ & $\mathrm{Cl} 7-\mathrm{Mo} 3-\mathrm{Mo} 1$ & $136.77(3)$ \\
\hline Mo3-Mo1-Mo2 & $60.038(12)$ & $\mathrm{Cl} 7-\mathrm{Mo} 3-\mathrm{Mo}^{\mathrm{i}}$ & $133.17(3)$ \\
\hline 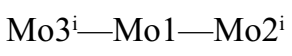 & $60.074(13)$ & $\mathrm{Cl} 7-\mathrm{Mo} 3-\mathrm{Mo} 2^{\mathrm{i}}$ & $135.56(3)$ \\
\hline Mo3 ${ }^{\mathrm{i}}-\mathrm{Mo} 1-\mathrm{Mo} 2$ & $60.036(15)$ & $\mathrm{Cl} 7-\mathrm{Mo} 3-\mathrm{Mo} 2$ & $134.21(3)$ \\
\hline Mo3 ${ }^{\mathrm{i}}-\mathrm{Mo} 1-\mathrm{Mo3}$ & $89.946(14)$ & $\mathrm{Cl} 7-\mathrm{Mo} 3-\mathrm{Cl} 1$ & $92.98(3)$ \\
\hline $\mathrm{Cl} 1-\mathrm{Mo} 1-\mathrm{Mo} 2^{\mathrm{i}}$ & $118.30(2)$ & $\mathrm{Cl} 7-\mathrm{Mo} 3-\mathrm{Cl}^{\mathrm{i}}$ & $91.41(3)$ \\
\hline $\mathrm{Cl} 1-\mathrm{Mo1}-\mathrm{Mo} 2$ & $58.24(2)$ & $\mathrm{Cl} 7-\mathrm{Mo} 3-\mathrm{Cl} 3$ & $93.96(3)$ \\
\hline $\mathrm{C} 11-\mathrm{Mo} 1-\mathrm{Mo}^{\mathrm{i}}$ & $118.26(2)$ & $\mathrm{Cl} 7-\mathrm{Mo} 3-\mathrm{Cl}^{\mathrm{i}}$ & $90.44(3)$ \\
\hline $\mathrm{Cl1}-\mathrm{Mo1}-\mathrm{Mo} 3$ & $58.37(2)$ & $\mathrm{Mo} 1-\mathrm{Cl1}-\mathrm{Mo} 2$ & $63.55(2)$ \\
\hline $\mathrm{Cl} 1-\mathrm{Mo} 1-\mathrm{Cl} 2$ & $89.61(3)$ & $\mathrm{Mo} 1-\mathrm{Cl1}-\mathrm{Mo} 3$ & $63.47(2)$ \\
\hline $\mathrm{Cl} 1-\mathrm{Mo} 1-\mathrm{Cl} 3$ & $90.01(3)$ & $\mathrm{Mo} 2-\mathrm{Cl1}-\mathrm{Mo} 3$ & $63.50(2)$ \\
\hline $\mathrm{Cl} 2-\mathrm{Mo} 1-\mathrm{Mo} 2$ & $57.97(2)$ & $\mathrm{Mo} 2-\mathrm{Cl} 2-\mathrm{Mo} 1$ & $63.61(2)$ \\
\hline $\mathrm{Cl} 2-\mathrm{Mo} 1-\mathrm{Mo}^{\mathrm{i}}$ & $118.37(2)$ & $\mathrm{Mo} 2-\mathrm{Cl} 2-\mathrm{Mo}^{\mathrm{i}}$ & $63.59(2)$ \\
\hline $\mathrm{Cl} 2-\mathrm{Mo} 1-\mathrm{Mo}^{\mathrm{i}}$ & $58.32(2)$ & $\mathrm{Mo} 3{ }^{\mathrm{i}}-\mathrm{Cl} 2-\mathrm{Mo} 1$ & $63.31(2)$ \\
\hline $\mathrm{Cl} 2-\mathrm{Mo} 1-\mathrm{Mo} 3$ & $117.99(2)$ & $\mathrm{Mo} 2 \mathrm{i}-\mathrm{Cl} 3-\mathrm{Mo} 1$ & $63.68(2)$ \\
\hline $\mathrm{Cl} 3-\mathrm{Mo} 1-\mathrm{Mo}^{\mathrm{i}}$ & $58.08(2)$ & $\mathrm{Mo} 2-\mathrm{Cl} 3-\mathrm{Mo} 3$ & $63.58(2)$ \\
\hline
\end{tabular}




$$
\begin{aligned}
& \mathrm{Cl} 3-\mathrm{Mo} 1-\mathrm{Mo} 2 \\
& \mathrm{Cl} 3-\mathrm{Mo} 1-\mathrm{Mo}^{\mathrm{i}} \\
& \mathrm{Cl} 3-\mathrm{Mo} 1-\mathrm{Mo} 3 \\
& \mathrm{Cl} 3-\mathrm{Mo} 1-\mathrm{Cl} 2 \\
& \text { Cl4-Mo1-Mo2 }{ }^{\mathrm{i}} \\
& \text { Cl4-Mo1-Mo2 } \\
& \text { Cl4-Mo1-Mo3 } \\
& \text { Cl4-Mo1-Mo3 }{ }^{\mathrm{i}} \\
& \text { Cl4-Mo1-Cl1 } \\
& \mathrm{Cl} 4-\mathrm{Mo} 1-\mathrm{Cl} 2 \\
& \mathrm{Cl} 4-\mathrm{Mo} 1-\mathrm{Cl} 3 \\
& \text { C15-Mo1-Mo2 }{ }^{\mathrm{i}} \\
& \text { Cl5-Mo1-Mo2 } \\
& \text { C15-Mo1-Mo3 } \\
& \text { Cl5-Mo1-Mo3 }{ }^{\mathrm{i}} \\
& \text { Cl5-Mo1-Cl1 } \\
& \mathrm{Cl} 5-\mathrm{Mo} 1-\mathrm{Cl} 2 \\
& \mathrm{Cl} 5-\mathrm{Mo} 1-\mathrm{Cl} 3 \\
& \text { Cl5-Mo1-Cl4 } \\
& \text { Mo1-Mo2-Mol }{ }^{\mathrm{i}} \\
& \text { Mo1-Mo2-Mo3 } \\
& \text { Mo3 }{ }^{\mathrm{i}}-\mathrm{Mo2}-\mathrm{Mol}^{\mathrm{i}} \\
& \text { Mo3 - Mo2-Mo1 } \\
& \text { Mo3-Mo2-Mo1 }{ }^{\mathrm{i}} \\
& \text { Mo3 }-\mathrm{Mo} 2-\mathrm{Mo} 3 \\
& \text { Cl1-Mo2-Mo1 }{ }^{\mathrm{i}} \\
& \mathrm{Cl1}-\mathrm{Mo2}-\mathrm{Mo} 1 \\
& \mathrm{Cl1}-\mathrm{Mo} 2-\mathrm{Mo}^{\mathrm{i}} \\
& \text { Cl1-Mo2-Mo3 } \\
& \mathrm{Cl} 2-\mathrm{Mo} 2-\mathrm{Mol}^{\mathrm{i}} \\
& \mathrm{Cl} 2-\mathrm{Mo} 2-\mathrm{Mo} 1 \\
& \mathrm{Cl} 2-\mathrm{Mo} 2-\mathrm{Mo} 3 \\
& \mathrm{Cl} 2-\mathrm{Mo} 2-\mathrm{Mo}^{\mathrm{i}} \\
& \mathrm{Cl} 2-\mathrm{Mo} 2-\mathrm{Cl} 1 \\
& \mathrm{Cl} 2-\mathrm{Mo} 2-\mathrm{Cl}^{\mathrm{i}} \\
& \mathrm{Cl} 3{ }^{\mathrm{i}}-\mathrm{Mo} 2-\mathrm{Mol}^{\mathrm{i}} \\
& \mathrm{Cl} 3 \text { - }-\mathrm{Mo} 2-\mathrm{Mo} 1 \\
& \mathrm{Cl} 3{ }^{\mathrm{i}}-\mathrm{Mo} 2-\mathrm{Mo}^{\mathrm{i}} \\
& \mathrm{Cl}^{3}-\mathrm{Mo} 2-\mathrm{Mo} 3 \\
& \mathrm{Cl} 3-\mathrm{Mo} 2-\mathrm{Cl} 1 \\
& \text { Cl4-Mo2-Mo1 } \\
& \mathrm{Cl} 1{ }^{\mathrm{i}}-\mathrm{Mo} 2-\mathrm{Mo}^{\mathrm{i}} \\
& \mathrm{Cl} 1 \text { - }-\mathrm{Mo} 2-\mathrm{Mo} 3 \\
& \mathrm{Cl} 4-\mathrm{Mo} 2-\mathrm{Mo}^{\mathrm{i}} \\
& \text { Cl4 - } \mathrm{Mo} 2-\mathrm{Cl} 1 \\
& \mathrm{Cl} 4-\mathrm{Mo} 2-\mathrm{Cl} 2 \\
& \mathrm{Cl} 4-\mathrm{Mo} 2-\mathrm{Cl}^{\mathrm{i}} \\
& \mathrm{Cl} 6-\mathrm{Mo} 2-\mathrm{Mo}^{\mathrm{i}}
\end{aligned}
$$

$118.24(2)$

118.13 (2)

$58.22(2)$

$175.50(3)$

$57.96(2)$

$118.09(2)$

$117.88(2)$

$58.08(2)$

$175.46(3)$

$90.28(3)$

$89.74(3)$

$134.38(3)$

135.49 (3)

$135.18(2)$

134.87 (3)

$92.58(3)$

92.57 (3)

$91.93(3)$

$91.96(3)$

$89.873(16)$

$59.978(12)$

$59.981(14)$

$59.875(13)$

$59.809(14)$

$89.819(14)$

118.14 (2)

$58.22(2)$

118.08 (2)

$58.34(2)$

118.37 (2)

$58.42(2)$

$118.38(2)$

58.40 (2)

$89.88(3)$

$89.95(3)$

$58.25(2)$

118.15 (2)

$58.29(2)$

118.04 (2)

$175.56(3)$

118.04 (2)

$58.26(2)$

58.07 (2)

$118.22(2)$

$89.81(3)$

$175.77(3)$

$90.03(3)$

133.87 (3)
$\mathrm{Mo} 3-\mathrm{Cl} 3-\mathrm{Mo} 1$

$\mathrm{Mo} 2$ - $\mathrm{Cl} 4-\mathrm{Mo} 1$

$\mathrm{Mo2}-\mathrm{Cl} 4-\mathrm{Mo}^{\mathrm{i}}$

Mo3 - $\mathrm{Cl} 4-\mathrm{Mo} 1$

H1A-N1-H1B

H1A-N1-H1C

H1B-N1-H1C

C1-N1-H1A

C1-N1-H1B

$\mathrm{C} 1-\mathrm{N} 1-\mathrm{H} 1 \mathrm{C}$

$\mathrm{C} 2-\mathrm{C} 1-\mathrm{N} 1$

$\mathrm{C} 2-\mathrm{C} 1-\mathrm{C} 3$

$\mathrm{C} 2 \mathrm{~A}-\mathrm{C} 1-\mathrm{N} 1$

$\mathrm{C} 2 \mathrm{~A}-\mathrm{C} 1-\mathrm{C} 3 \mathrm{~A}$

$\mathrm{C} 3-\mathrm{C} 1-\mathrm{N} 1$

$\mathrm{C} 3 \mathrm{~A}-\mathrm{C} 1-\mathrm{N} 1$

$\mathrm{C} 1-\mathrm{C} 2-\mathrm{H} 2$

$\mathrm{C} 1-\mathrm{C} 2-\mathrm{C} 3^{\mathrm{ii}}$

$\mathrm{C} 3$ ii- $22-\mathrm{H} 2$

$\mathrm{C} 1-\mathrm{C} 2 \mathrm{~A}-\mathrm{H} 2 \mathrm{~A}$

$\mathrm{C} 1-\mathrm{C} 2 \mathrm{~A}-\mathrm{C} 3 \mathrm{~A}^{\mathrm{ii}}$

$\mathrm{C} 3 \mathrm{~A}^{\mathrm{ii}}-\mathrm{C} 2 \mathrm{~A}-\mathrm{H} 2 \mathrm{~A}$

$\mathrm{C} 1-\mathrm{C} 3-\mathrm{C}^{\mathrm{ii}}$

$\mathrm{C} 1-\mathrm{C} 3-\mathrm{H} 3$

$\mathrm{C} 2{ }^{\mathrm{ii}}-\mathrm{C} 3-\mathrm{H} 3$

$\mathrm{C} 1-\mathrm{C} 3 \mathrm{~A}-\mathrm{C} 2 \mathrm{~A}^{\mathrm{ii}}$

$\mathrm{C} 1-\mathrm{C} 3 \mathrm{~A}-\mathrm{H} 3 \mathrm{~A}$

$\mathrm{C} 2 \mathrm{~A} i \mathrm{ii} 3 \mathrm{~A}-\mathrm{H} 3 \mathrm{~A}$

$\mathrm{C} 5-\mathrm{N} 2-\mathrm{C} 4$

$\mathrm{C} 6-\mathrm{N} 2-\mathrm{C} 4$

$\mathrm{C} 6-\mathrm{N} 2-\mathrm{C} 5$

$\mathrm{N} 2-\mathrm{C} 4-\mathrm{H} 4 \mathrm{~A}$

$\mathrm{N} 2-\mathrm{C} 4-\mathrm{H} 4 \mathrm{~B}$

$\mathrm{N} 2-\mathrm{C} 4-\mathrm{H} 4 \mathrm{C}$

$\mathrm{H} 4 \mathrm{~A}-\mathrm{C} 4-\mathrm{H} 4 \mathrm{~B}$

$\mathrm{H} 4 \mathrm{~A}-\mathrm{C} 4-\mathrm{H} 4 \mathrm{C}$

$\mathrm{H} 4 \mathrm{~B}-\mathrm{C} 4-\mathrm{H} 4 \mathrm{C}$

$\mathrm{N} 2-\mathrm{C} 5-\mathrm{H} 5 \mathrm{~A}$

$\mathrm{N} 2-\mathrm{C} 5-\mathrm{H} 5 \mathrm{~B}$

$\mathrm{N} 2-\mathrm{C} 5-\mathrm{H} 5 \mathrm{C}$

$\mathrm{H} 5 \mathrm{~A}-\mathrm{C} 5-\mathrm{H} 5 \mathrm{~B}$

$\mathrm{H} 5 \mathrm{~A}-\mathrm{C} 5-\mathrm{H} 5 \mathrm{C}$

$\mathrm{H} 5 \mathrm{~B}-\mathrm{C} 5-\mathrm{H} 5 \mathrm{C}$

$\mathrm{O} 1-\mathrm{C} 6-\mathrm{N} 2$

$\mathrm{O} 1-\mathrm{C} 6-\mathrm{H} 6$

N2-C6- 66

$\mathrm{C} 7-\mathrm{N} 3-\mathrm{C} 8$

$\mathrm{C} 9-\mathrm{N} 3-\mathrm{C} 7$
$63.55(2)$

$63.78(2)$

$63.86(2)$

$63.56(2)$

$112(4)$

110 (4)

$103(3)$

108 (3)

109 (3)

114 (3)

119.9 (3)

120.7 (4)

$119.6(15)$

$120.3(10)$

119.4 (3)

120.1 (16)

119.9

120.1 (4)

119.9

121.4

117 (3)

121.4

119.2 (4)

120.4

120.4

$122(3)$

118.8

118.8

$117.3(3)$

122.3 (3)

120.4 (3)

109.5

109.5

109.5

109.5

109.5

109.5

109.5

109.5

109.5

109.5

109.5

109.5

127.2 (4)

116.4

116.4

117.8 (4)

120.7 (4) 


\begin{tabular}{|c|c|c|c|}
\hline $\mathrm{Cl} 16-\mathrm{Mo} 2-\mathrm{Mo} 1$ & $136.26(3)$ & $\mathrm{C} 9-\mathrm{N} 3-\mathrm{C} 8$ & $121.5(4)$ \\
\hline $\mathrm{Cl} 6-\mathrm{Mo} 2-\mathrm{Mo}^{\mathrm{i}}$ & $134.96(3)$ & $\mathrm{N} 3-\mathrm{C} 7-\mathrm{H} 7 \mathrm{~A}$ & 109.5 \\
\hline $\mathrm{Cl} 6-\mathrm{Mo} 2-\mathrm{Mo} 3$ & $135.19(2)$ & $\mathrm{N} 3-\mathrm{C} 7-\mathrm{H} 7 \mathrm{~B}$ & 109.5 \\
\hline $\mathrm{Cl} 6-\mathrm{Mo} 2-\mathrm{Cl} 1$ & $93.14(3)$ & $\mathrm{N} 3-\mathrm{C} 7-\mathrm{H} 7 \mathrm{C}$ & 109.5 \\
\hline $\mathrm{Cl} 6-\mathrm{Mo} 2-\mathrm{Cl} 2$ & $92.74(3)$ & $\mathrm{H} 7 \mathrm{~A}-\mathrm{C} 7-\mathrm{H} 7 \mathrm{~B}$ & 109.5 \\
\hline $\mathrm{Cl} 6-\mathrm{Mo} 2-\mathrm{Cl}^{\mathrm{i}}$ & $91.30(3)$ & $\mathrm{H} 7 \mathrm{~A}-\mathrm{C} 7-\mathrm{H} 7 \mathrm{C}$ & 109.5 \\
\hline $\mathrm{Cl} 6-\mathrm{Mo} 2-\mathrm{Cl}_{4}^{\mathrm{i}}$ & $91.49(3)$ & $\mathrm{H} 7 \mathrm{~B}-\mathrm{C} 7-\mathrm{H} 7 \mathrm{C}$ & 109.5 \\
\hline Mo1-Mo3-Mo1 & $90.053(14)$ & $\mathrm{N} 3-\mathrm{C} 8-\mathrm{H} 8 \mathrm{~A}$ & 109.5 \\
\hline $\mathrm{Mo} 1^{\mathrm{i}}-\mathrm{Mo} 3-\mathrm{Mo}^{\mathrm{i}}$ & $60.086(12)$ & $\mathrm{N} 3-\mathrm{C} 8-\mathrm{H} 8 \mathrm{~B}$ & 109.5 \\
\hline Mo1-Mo3-Mo2 & $59.984(14)$ & $\mathrm{N} 3-\mathrm{C} 8-\mathrm{H} 8 \mathrm{C}$ & 109.5 \\
\hline Mo1 ${ }^{\mathrm{i}}-\mathrm{Mo} 3-\mathrm{Mo} 2$ & $60.116(12)$ & $\mathrm{H} 8 \mathrm{~A}-\mathrm{C} 8-\mathrm{H} 8 \mathrm{~B}$ & 109.5 \\
\hline $\mathrm{Mo2}-\mathrm{Mo} 3-\mathrm{Mo1}$ & $60.082(11)$ & $\mathrm{H} 8 \mathrm{~A}-\mathrm{C} 8-\mathrm{H} 8 \mathrm{C}$ & 109.5 \\
\hline $\mathrm{Mo} 2 \mathrm{i}-\mathrm{Mo} 3-\mathrm{Mo} 2$ & $90.179(13)$ & $\mathrm{H} 8 \mathrm{~B}-\mathrm{C} 8-\mathrm{H} 8 \mathrm{C}$ & 109.5 \\
\hline $\mathrm{Cl1}-\mathrm{Mo} 3-\mathrm{Mo1}^{\mathrm{i}}$ & $118.25(2)$ & $\mathrm{O} 2-\mathrm{C} 9-\mathrm{N} 3$ & $126.1(4)$ \\
\hline Cl1-Mo3-Mo1 & $58.16(2)$ & $\mathrm{O} 2-\mathrm{C} 9-\mathrm{H} 9$ & 116.9 \\
\hline C11-Mo3-Mo2 & $58.15(2)$ & $\mathrm{N} 3-\mathrm{C} 9-\mathrm{H} 9$ & 116.9 \\
\hline $\mathrm{C} 11-\mathrm{Mo} 3-\mathrm{Mo} 2^{\mathrm{i}}$ & $118.23(2)$ & $\mathrm{C} 11-\mathrm{N} 4-\mathrm{C} 10$ & $117.9(3)$ \\
\hline $\mathrm{Cl} 2^{\mathrm{i}}-\mathrm{Mo} 3-\mathrm{Mo} 1$ & $118.07(2)$ & $\mathrm{C} 12-\mathrm{N} 4-\mathrm{C} 10$ & $121.7(3)$ \\
\hline $\mathrm{Cl} 2^{\mathrm{i}}-\mathrm{Mo} 3-\mathrm{Mo}^{\mathrm{i}}$ & $58.37(2)$ & $\mathrm{C} 12-\mathrm{N} 4-\mathrm{C} 11$ & $120.3(4)$ \\
\hline $\mathrm{Cl} 2-\mathrm{Mo} 3-\mathrm{Mo} 2$ & $118.46(2)$ & $\mathrm{N} 4-\mathrm{C} 10-\mathrm{H} 10 \mathrm{~A}$ & 109.5 \\
\hline $\mathrm{Cl} 2^{\mathrm{i}}-\mathrm{Mo} 3-\mathrm{Mo} 2^{\mathrm{i}}$ & $58.00(2)$ & $\mathrm{N} 4-\mathrm{C} 10-\mathrm{H} 10 \mathrm{~B}$ & 109.5 \\
\hline $\mathrm{Cl} 2 \mathrm{i}-\mathrm{Mo} 3-\mathrm{Cl} 1$ & $175.60(3)$ & $\mathrm{N} 4-\mathrm{C} 10-\mathrm{H} 10 \mathrm{C}$ & 109.5 \\
\hline $\mathrm{Cl} 3-\mathrm{Mo} 3-\mathrm{Mo} 1$ & $58.23(2)$ & $\mathrm{H} 10 \mathrm{~A}-\mathrm{C} 10-\mathrm{H} 10 \mathrm{~B}$ & 109.5 \\
\hline $\mathrm{Cl} 3-\mathrm{Mo} 3-\mathrm{Mo}^{\mathrm{i}}$ & $118.20(2)$ & $\mathrm{H} 10 \mathrm{~A}-\mathrm{C} 10-\mathrm{H} 10 \mathrm{C}$ & 109.5 \\
\hline $\mathrm{Cl} 3-\mathrm{Mo} 3-\mathrm{Mo} 2$ & $118.20(2)$ & $\mathrm{H} 10 \mathrm{~B}-\mathrm{C} 10-\mathrm{H} 10 \mathrm{C}$ & 109.5 \\
\hline $\mathrm{Cl} 3-\mathrm{Mo} 3-\mathrm{Mo} 2^{\mathrm{i}}$ & $58.13(2)$ & $\mathrm{N} 4-\mathrm{C} 11-\mathrm{H} 11 \mathrm{~A}$ & 109.5 \\
\hline $\mathrm{Cl} 3-\mathrm{Mo} 3-\mathrm{Cl} 1$ & $89.89(3)$ & $\mathrm{N} 4-\mathrm{C} 11-\mathrm{H} 11 \mathrm{~B}$ & 109.5 \\
\hline $\mathrm{Cl} 3-\mathrm{Mo} 3-\mathrm{Cl} 2^{\mathrm{i}}$ & $89.61(3)$ & $\mathrm{N} 4-\mathrm{C} 11-\mathrm{H} 11 \mathrm{C}$ & 109.5 \\
\hline $\mathrm{Cl} 4-\mathrm{Mo} 3-\mathrm{Mo} 1$ & $118.03(2)$ & $\mathrm{H} 11 \mathrm{~A}-\mathrm{C} 11-\mathrm{H} 11 \mathrm{~B}$ & 109.5 \\
\hline $\mathrm{Cl} 4-\mathrm{Mo} 3-\mathrm{Mo}^{\mathrm{i}}$ & $58.36(2)$ & $\mathrm{H} 11 \mathrm{~A}-\mathrm{C} 11-\mathrm{H} 11 \mathrm{C}$ & 109.5 \\
\hline $\mathrm{Cl} 4^{\mathrm{i}}-\mathrm{Mo} 3-\mathrm{Mo} 2^{\mathrm{i}}$ & $118.43(2)$ & $\mathrm{H} 11 \mathrm{~B}-\mathrm{C} 11-\mathrm{H} 11 \mathrm{C}$ & 109.5 \\
\hline $\mathrm{Cl} 4 \mathrm{i}-\mathrm{Mo} 3-\mathrm{Mo} 2$ & $58.06(2)$ & $\mathrm{O} 3-\mathrm{C} 12-\mathrm{N} 4$ & $125.0(4)$ \\
\hline $\mathrm{Cl} 4-\mathrm{Mo} 3-\mathrm{Cl} 1$ & $89.68(3)$ & $\mathrm{O} 3-\mathrm{C} 12-\mathrm{H} 12$ & 117.5 \\
\hline $\mathrm{Cl} 4^{\mathrm{i}}-\mathrm{Mo} 3-\mathrm{Cl}^{\mathrm{i}}$ & $90.48(3)$ & $\mathrm{N} 4-\mathrm{C} 12-\mathrm{H} 12$ & 117.5 \\
\hline $\mathrm{N} 1-\mathrm{C} 1-\mathrm{C} 2-\mathrm{C} 3^{\mathrm{ii}}$ & $178.5(3)$ & $\mathrm{C} 3 \mathrm{~A}-\mathrm{C} 1-\mathrm{C} 2 \mathrm{~A}-\mathrm{C} 3 \mathrm{~A}^{\mathrm{ii}}$ & $1.0(17)$ \\
\hline $\mathrm{N} 1-\mathrm{C} 1-\mathrm{C} 2 \mathrm{~A}-\mathrm{C} 3 \mathrm{~A}^{\mathrm{ii}}$ & $-179.7(9)$ & $\mathrm{C} 4-\mathrm{N} 2-\mathrm{C} 6-\mathrm{O} 1$ & $177.0(4)$ \\
\hline $\mathrm{N} 1-\mathrm{C} 1-\mathrm{C} 3-\mathrm{C} 2^{\mathrm{ii}}$ & $-178.5(3)$ & $\mathrm{C} 5-\mathrm{N} 2-\mathrm{C} 6-\mathrm{O} 1$ & $-1.6(6)$ \\
\hline $\mathrm{N} 1-\mathrm{C} 1-\mathrm{C} 3 \mathrm{~A}-\mathrm{C} 2 \mathrm{~A}^{\mathrm{ii}}$ & $179.7(9)$ & $\mathrm{C} 7-\mathrm{N} 3-\mathrm{C} 9-\mathrm{O} 2$ & $-2.6(6)$ \\
\hline $\mathrm{C} 2-\mathrm{C} 1-\mathrm{C} 3-\mathrm{C} 2^{\mathrm{ii}}$ & $0.3(6)$ & $\mathrm{C} 8-\mathrm{N} 3-\mathrm{C} 9-\mathrm{O} 2$ & $179.8(4)$ \\
\hline $\mathrm{C} 2 \mathrm{~A}-\mathrm{C} 1-\mathrm{C} 3 \mathrm{~A}-\mathrm{C} 2 \mathrm{~A}^{\mathrm{ii}}$ & $-1.1(18)$ & $\mathrm{C} 10-\mathrm{N} 4-\mathrm{C} 12-\mathrm{O} 3$ & $177.3(4)$ \\
\hline $\mathrm{C} 3-\mathrm{C} 1-\mathrm{C} 2-\mathrm{C}^{3 i}$ & $-0.3(6)$ & $\mathrm{C} 11-\mathrm{N} 4-\mathrm{C} 12-\mathrm{O} 3$ & $0.4(6)$ \\
\hline
\end{tabular}

Symmetry codes: (i) $-x+1,-y+1,-z+1$; (ii) $-x+1,-y+1,-z$.

Hydrogen-bond geometry $\left(A,{ }^{\circ}\right)$

\begin{tabular}{lllll}
\hline$D-\mathrm{H} \cdots A$ & $D-\mathrm{H}$ & $\mathrm{H} \cdots A$ & $D \cdots A$ & $D-\mathrm{H} \cdots A$ \\
\hline $\mathrm{N} 1-\mathrm{H} 1 A \cdots \mathrm{O} 2^{\mathrm{iii}}$ & $0.92(2)$ & $1.76(2)$ & $2.672(4)$ & $171(4)$
\end{tabular}




$\begin{array}{lllll}\mathrm{N} 1-\mathrm{H} 1 B \cdots \mathrm{O} 3^{\text {iv }} & 0.93(2) & 1.79(2) & 2.710(4) & 173(4) \\ \mathrm{N} 1-\mathrm{H} 1 C \cdots{ }^{\mathrm{i}} & 0.92(2) & 1.81(2) & 2.727(4) & 175(4)\end{array}$

Symmetry codes: (i) $-x+1,-y+1,-z+1$; (iii) $x, y+1, z-1$; (iv) $-x+2,-y+1,-z$.

$N, N^{\prime}$-(1,4-Phenylene)bis(propan-2-iminium) octa- $\mu_{3}$-chlorido-hexachlorido-octahedro-hexamolybdate acetone trisolvate (3)

Crystal data

$\left(\mathrm{C}_{12} \mathrm{H}_{18} \mathrm{~N}_{2}\right)\left[\mathrm{Mo}_{6} \mathrm{Cl}_{8} \mathrm{Cl}_{6}\right] \cdot 3 \mathrm{C}_{3} \mathrm{H}_{6} \mathrm{O}$

$Z=1$

$M_{r}=1436.46$

$F(000)=690$

Triclinic, $P \overline{1}$

$a=9.451(2) \AA$

$b=11.236(3) \AA$

$c=11.712(3) \AA$

$\alpha=64.933(6)^{\circ}$

$\beta=71.174(6)^{\circ}$

$\gamma=75.440(6)^{\circ}$

$D_{\mathrm{x}}=2.257 \mathrm{Mg} \mathrm{m}^{-3}$

Mo $K \alpha$ radiation, $\lambda=0.71073 \AA$

$V=1056.7(5) \AA^{3}$

Cell parameters from 5309 reflections

$\theta=2.2-25.1^{\circ}$

$\mu=2.64 \mathrm{~mm}^{-1}$

$T=200 \mathrm{~K}$

Block, clear light orange

$0.55 \times 0.33 \times 0.20 \mathrm{~mm}$

\section{Data collection}

Bruker SMART X2S benchtop diffractometer

Radiation source: sealed microfocus source, XOS X-beam microfocus source

Graphite monochromator

Detector resolution: 8.3330 pixels $\mathrm{mm}^{-1}$

$\varphi$ and $\omega$ scans

Absorption correction: multi-scan

(SADABS; Bruker, 2012)

$T_{\min }=0.490, T_{\max }=0.745$

10036 measured reflections

3692 independent reflections

3220 reflections with $I>2 \sigma(I)$

$R_{\text {int }}=0.030$

$\theta_{\max }=25.2^{\circ}, \theta_{\min }=2.6^{\circ}$

$h=-11 \rightarrow 11$

$k=-13 \rightarrow 13$

$l=-13 \rightarrow 13$

Refinement

Refinement on $F^{2}$

Least-squares matrix: full

$R\left[F^{2}>2 \sigma\left(F^{2}\right)\right]=0.025$

$w R\left(F^{2}\right)=0.068$

$S=1.05$

3692 reflections

235 parameters

13 restraints

Primary atom site location: heavy-atom method

Hydrogen site location: mixed

$\mathrm{H}$ atoms treated by a mixture of independent and constrained refinement

$w=1 /\left[\sigma^{2}\left(F_{\mathrm{o}}^{2}\right)+(0.0347 P)^{2}+0.3989 P\right]$

where $P=\left(F_{\mathrm{o}}^{2}+2 F_{\mathrm{c}}^{2}\right) / 3$

$(\Delta / \sigma)_{\max }=0.001$

$\Delta \rho_{\max }=0.96 \mathrm{e} \AA^{-3}$

$\Delta \rho_{\min }=-0.82$ e $\AA^{-3}$

Special details

Geometry. All esds (except the esd in the dihedral angle between two 1.s. planes) are estimated using the full covariance matrix. The cell esds are taken into account individually in the estimation of esds in distances, angles and torsion angles; correlations between esds in cell parameters are only used when they are defined by crystal symmetry. An approximate (isotropic) treatment of cell esds is used for estimating esds involving l.s. planes.

Fractional atomic coordinates and isotropic or equivalent isotropic displacement parameters $\left(\hat{A}^{2}\right)$

\begin{tabular}{llllll}
\hline & $x$ & $y$ & $z$ & $U_{\text {iso }} * / U_{\text {eq }}$ & Occ. $(<1)$ \\
\hline Mo1 & $0.54044(3)$ & $0.46936(2)$ & $0.65449(2)$ & $0.01669(9)$ & \\
Mo2 & $0.46251(3)$ & $0.32776(2)$ & $0.56169(2)$ & $0.01681(9)$ & \\
Mo3 & $0.29965(3)$ & $0.55064(2)$ & $0.56544(2)$ & $0.01718(9)$ &
\end{tabular}




\begin{tabular}{|c|c|c|c|c|c|}
\hline $\mathrm{Cl1}$ & $0.41212(10)$ & $0.10003(8)$ & $0.64118(8)$ & $0.0324(2)$ & \\
\hline $\mathrm{Cl} 2$ & $0.76390(8)$ & $0.58538(7)$ & $0.52537(7)$ & $0.02273(17)$ & \\
\hline $\mathrm{Cl} 3$ & $0.69229(9)$ & $0.25958(7)$ & $0.64379(7)$ & $0.02413(18)$ & \\
\hline $\mathrm{Cl} 4$ & $0.59212(10)$ & $0.43277(8)$ & $0.85707(7)$ & $0.0303(2)$ & \\
\hline $\mathrm{Cl} 5$ & $0.38283(9)$ & $0.68264(7)$ & $0.65005(7)$ & $0.02236(17)$ & \\
\hline $\mathrm{Cl} 6$ & $0.31409(9)$ & $0.35417(8)$ & $0.76707(7)$ & $0.02377(18)$ & \\
\hline $\mathrm{Cl} 7$ & $0.03772(9)$ & $0.61748(9)$ & $0.65222(9)$ & $0.0360(2)$ & \\
\hline N1 & $0.2062(3)$ & $0.0615(3)$ & $0.2565(3)$ & $0.0242(6)$ & \\
\hline $\mathrm{H} 1$ & $0.196(4)$ & $0.021(3)$ & $0.211(3)$ & $0.036^{*}$ & \\
\hline $\mathrm{C} 1$ & $0.4140(4)$ & $0.1369(4)$ & $0.0787(3)$ & $0.0382(9)$ & \\
\hline H1A & 0.431717 & 0.228439 & 0.022574 & $0.057 *$ & \\
\hline H1B & 0.366916 & 0.102550 & 0.037660 & $0.057 *$ & \\
\hline $\mathrm{H} 1 \mathrm{C}$ & 0.510355 & 0.081919 & 0.091601 & $0.057 *$ & \\
\hline $\mathrm{C} 2$ & $0.3131(4)$ & $0.1337(3)$ & $0.2058(3)$ & $0.0265(7)$ & \\
\hline $\mathrm{C} 3$ & $0.3406(4)$ & $0.2109(3)$ & $0.2704(4)$ & $0.0370(9)$ & \\
\hline $\mathrm{H} 3 \mathrm{~A}$ & 0.424810 & 0.163487 & 0.311157 & $0.056^{*}$ & \\
\hline $\mathrm{H} 3 \mathrm{~B}$ & 0.249770 & 0.222227 & 0.337134 & $0.056^{*}$ & \\
\hline $\mathrm{H} 3 \mathrm{C}$ & 0.365572 & 0.298048 & 0.205817 & $0.056^{*}$ & \\
\hline $\mathrm{C} 4$ & $0.1507(4)$ & $-0.0037(3)$ & $0.4928(3)$ & $0.0281(7)$ & \\
\hline $\mathrm{H} 4$ & 0.253881 & -0.006002 & 0.486934 & $0.034 *$ & \\
\hline $\mathrm{C} 5$ & $0.1019(4)$ & $0.0328(3)$ & $0.3819(3)$ & $0.0227(7)$ & \\
\hline C6 & $-0.0482(4)$ & $0.0368(3)$ & $0.3885(3)$ & $0.0274(7)$ & \\
\hline H6 & -0.079966 & 0.062241 & 0.311317 & $0.033^{*}$ & \\
\hline $\mathrm{O} 1$ & $0.1737(3)$ & $0.9573(3)$ & $0.0897(2)$ & $0.0440(7)$ & \\
\hline $\mathrm{C} 7$ & $0.0245(5)$ & $0.7839(4)$ & $0.1942(4)$ & $0.0502(11)$ & \\
\hline H7A & 0.012696 & 0.802221 & 0.271696 & $0.075^{*}$ & \\
\hline $\mathrm{H} 7 \mathrm{~B}$ & 0.067673 & 0.691137 & 0.209401 & $0.075^{*}$ & \\
\hline $\mathrm{H} 7 \mathrm{C}$ & -0.074289 & 0.800190 & 0.175911 & $0.075^{*}$ & \\
\hline $\mathrm{C} 8$ & $0.1262(4)$ & $0.8717(4)$ & $0.0818(4)$ & $0.0345(8)$ & \\
\hline C9 & $0.1705(5)$ & $0.8501(4)$ & $-0.0432(4)$ & $0.0468(10)$ & \\
\hline H9A & 0.205823 & 0.930184 & -0.115350 & $0.070 *$ & \\
\hline H9B & 0.083109 & 0.830392 & -0.056688 & $0.070^{*}$ & \\
\hline $\mathrm{H} 9 \mathrm{C}$ & 0.251527 & 0.775370 & -0.039653 & $0.070^{*}$ & \\
\hline $\mathrm{O} 2$ & $0.0767(6)$ & $0.3001(5)$ & $0.0578(5)$ & $0.0461(13)$ & 0.5 \\
\hline $\mathrm{C} 10$ & $0.0375(9)$ & $0.4167(7)$ & $0.0246(6)$ & $0.0351(17)$ & 0.5 \\
\hline $\mathrm{C} 11$ & $0.143(2)$ & $0.512(2)$ & $-0.011(2)$ & $0.049(5)$ & 0.5 \\
\hline H11A & 0.117815 & 0.548863 & 0.056809 & $0.073^{*}$ & 0.5 \\
\hline H11B & 0.134909 & 0.584134 & -0.094095 & $0.073^{*}$ & 0.5 \\
\hline $\mathrm{H} 11 \mathrm{C}$ & 0.246860 & 0.466249 & -0.018685 & $0.073^{*}$ & 0.5 \\
\hline $\mathrm{C} 12$ & $-0.1156(17)$ & $0.473(2)$ & $0.006(2)$ & $0.044(5)$ & 0.5 \\
\hline $\mathrm{H} 12 \mathrm{~A}$ & -0.114886 & 0.495968 & -0.085108 & $0.067^{*}$ & 0.5 \\
\hline H12B & -0.148442 & 0.553655 & 0.026059 & $0.067 *$ & 0.5 \\
\hline $\mathrm{H} 12 \mathrm{C}$ & -0.185388 & 0.408443 & 0.063119 & $0.067^{*}$ & 0.5 \\
\hline
\end{tabular}

Atomic displacement parameters $\left(\AA^{2}\right)$

\begin{tabular}{lllllll}
\hline & $U^{11}$ & $U^{22}$ & $U^{33}$ & $U^{12}$ & $U^{13}$ & $U^{23}$ \\
\hline Mo1 & $0.01921(17)$ & $0.01543(15)$ & $0.01644(15)$ & $-0.00245(12)$ & $-0.00747(11)$ & $-0.00459(11)$
\end{tabular}




$\begin{array}{lllllll}\text { Mo2 } & 0.01936(17) & 0.01344(15) & 0.01826(15) & -0.00251(11) & -0.00714(11) & -0.00450(11) \\ \text { Mo3 } & 0.01548(17) & 0.01715(15) & 0.01989(15) & -0.00117(11) & -0.00588(11) & -0.00728(12) \\ \text { C11 } & 0.0385(5) & 0.0179(4) & 0.0390(5) & -0.0077(4) & -0.0110(4) & -0.0057(3) \\ \text { C12 } & 0.0208(4) & 0.0243(4) & 0.0272(4) & -0.0053(3) & -0.0092(3) & -0.0098(3) \\ \text { C13 } & 0.0278(4) & 0.0172(4) & 0.0286(4) & 0.0030(3) & -0.0158(3) & -0.0065(3) \\ \text { C14 } & 0.0429(5) & 0.0301(4) & 0.0223(4) & -0.0098(4) & -0.0159(4) & -0.0053(3) \\ \text { C15 } & 0.0248(4) & 0.0208(4) & 0.0247(4) & -0.0008(3) & -0.0075(3) & -0.0118(3) \\ \text { C16 } & 0.0266(4) & 0.0245(4) & 0.0186(4) & -0.0084(3) & -0.0029(3) & -0.0057(3) \\ \text { C17 } & 0.0189(4) & 0.0453(5) & 0.0457(5) & 0.0015(4) & -0.0055(4) & -0.0237(4) \\ \text { N1 } & 0.0263(16) & 0.0219(14) & 0.0257(14) & -0.0046(12) & -0.0068(12) & -0.0090(11) \\ \text { C1 } & 0.040(2) & 0.036(2) & 0.0310(19) & -0.0163(18) & -0.0023(16) & -0.0037(16) \\ \text { C2 } & 0.0241(19) & 0.0158(16) & 0.0354(18) & 0.0018(14) & -0.0126(15) & -0.0045(14) \\ \text { C3 } & 0.037(2) & 0.0235(18) & 0.054(2) & -0.0030(16) & -0.0155(18) & -0.0152(17) \\ \text { C4 } & 0.0213(18) & 0.0312(18) & 0.0346(18) & -0.0018(15) & -0.0099(15) & -0.0136(15) \\ \text { C5 } & 0.0251(19) & 0.0163(15) & 0.0275(16) & 0.0008(13) & -0.0074(14) & -0.0102(13) \\ \text { C6 } & 0.028(2) & 0.0311(18) & 0.0265(17) & -0.0021(15) & -0.0113(14) & -0.0115(15) \\ \text { O1 } & 0.0565(18) & 0.0433(16) & 0.0429(15) & -0.0198(14) & -0.0152(13) & -0.0166(13) \\ \text { C7 } & 0.038(2) & 0.047(2) & 0.063(3) & -0.012(2) & -0.013(2) & -0.014(2) \\ \text { C8 } & 0.031(2) & 0.0322(19) & 0.043(2) & 0.0004(16) & -0.0204(17) & -0.0105(16) \\ \text { C9 } & 0.057(3) & 0.043(2) & 0.050(2) & -0.001(2) & -0.028(2) & -0.018(2) \\ \text { O2 } & 0.051(4) & 0.037(3) & 0.052(3) & -0.007(3) & -0.016(3) & -0.015(3) \\ \text { C10 } & 0.046(5) & 0.038(5) & 0.020(3) & -0.006(4) & -0.003(3) & -0.013(3) \\ \text { C11 } & 0.048(8) & 0.054(10) & 0.061(10) & -0.010(7) & -0.008(8) & -0.040(8) \\ \text { C12 } & 0.043(7) & 0.042(8) & 0.036(8) & 0.000(6) & 0.006(5) & -0.017(7) \\ & & & & & & \end{array}$

Geometric parameters $\left(\hat{A},{ }^{o}\right)$

\begin{tabular}{|c|c|c|c|}
\hline $\mathrm{Mo} 1-\mathrm{Mo}^{\mathrm{i}}$ & $2.5943(6)$ & $\mathrm{C} 2-\mathrm{C} 3$ & $1.480(5)$ \\
\hline Mo1-Mo2 & $2.6126(5)$ & $\mathrm{C} 3-\mathrm{H} 3 \mathrm{~A}$ & 0.9800 \\
\hline Mo1-Mo3 & $2.6038(6)$ & $\mathrm{C} 3-\mathrm{H} 3 \mathrm{~B}$ & 0.9800 \\
\hline Mo1-Mo3 ${ }^{\mathrm{i}}$ & $2.6031(7)$ & $\mathrm{C} 3-\mathrm{H} 3 \mathrm{C}$ & 0.9800 \\
\hline $\mathrm{Mo} 1-\mathrm{Cl} 2$ & $2.4695(9)$ & $\mathrm{C} 4-\mathrm{H} 4$ & 0.9500 \\
\hline $\mathrm{Mo} 1-\mathrm{Cl} 3$ & $2.4627(9)$ & $\mathrm{C} 4-\mathrm{C} 5$ & $1.381(4)$ \\
\hline Mo1-Cl4 & $2.4202(9)$ & $\mathrm{C} 4-\mathrm{C}^{6 i}$ & $1.372(5)$ \\
\hline $\mathrm{Mo} 1-\mathrm{Cl} 5$ & $2.4727(9)$ & $\mathrm{C} 5-\mathrm{C} 6$ & $1.386(4)$ \\
\hline Mo1-Cl6 & $2.4729(9)$ & $\mathrm{C} 6-\mathrm{H} 6$ & 0.9500 \\
\hline Mo2-Mo3 ${ }^{i}$ & $2.6069(6)$ & $\mathrm{O} 1-\mathrm{C} 8$ & $1.206(4)$ \\
\hline Mo2-Mo3 & $2.5989(7)$ & $\mathrm{C} 7-\mathrm{H} 7 \mathrm{~A}$ & 0.9800 \\
\hline $\mathrm{Mo} 2-\mathrm{Cl1}$ & $2.4391(10)$ & C7-H7B & 0.9800 \\
\hline $\mathrm{Mo} 2-\mathrm{Cl} 2^{\mathrm{i}}$ & $2.4668(9)$ & $\mathrm{C} 7-\mathrm{H} 7 \mathrm{C}$ & 0.9800 \\
\hline $\mathrm{Mo} 2-\mathrm{Cl} 3$ & $2.4727(9)$ & $\mathrm{C} 7-\mathrm{C} 8$ & $1.480(5)$ \\
\hline $\mathrm{Mo} 2-\mathrm{Cl}^{\mathrm{i}}$ & $2.4738(9)$ & $\mathrm{C} 8-\mathrm{C} 9$ & $1.494(5)$ \\
\hline $\mathrm{Mo} 2-\mathrm{Cl} 6$ & $2.4616(9)$ & C9-H9A & 0.9800 \\
\hline $\mathrm{Mo} 3-\mathrm{Cl} 2^{\mathrm{i}}$ & $2.4655(8)$ & C9-H9B & 0.9800 \\
\hline $\mathrm{Mo} 3-\mathrm{Cl}^{\mathrm{i}}$ & $2.4680(9)$ & $\mathrm{C} 9-\mathrm{H} 9 \mathrm{C}$ & 0.9800 \\
\hline $\mathrm{Mo} 3-\mathrm{Cl} 5$ & $2.4767(8)$ & $\mathrm{O} 2-\mathrm{C} 10$ & $1.193(8)$ \\
\hline $\mathrm{Mo} 3-\mathrm{Cl} 6$ & $2.4714(9)$ & $\mathrm{C} 10-\mathrm{C} 11$ & $1.488(13)$ \\
\hline $\mathrm{Mo} 3-\mathrm{Cl} 7$ & $2.4110(10)$ & $\mathrm{C} 10-\mathrm{C} 12$ & $1.475(13)$ \\
\hline
\end{tabular}




\begin{tabular}{|c|c|c|c|}
\hline $\mathrm{N} 1-\mathrm{H} 1$ & $0.870(18)$ & $\mathrm{C} 11-\mathrm{H} 11 \mathrm{~A}$ & 0.9800 \\
\hline $\mathrm{N} 1-\mathrm{C} 2$ & $1.284(4)$ & $\mathrm{C} 11-\mathrm{H} 11 \mathrm{~B}$ & 0.9800 \\
\hline $\mathrm{N} 1-\mathrm{C} 5$ & $1.434(4)$ & $\mathrm{C} 11-\mathrm{H} 11 \mathrm{C}$ & 0.9800 \\
\hline $\mathrm{C} 1-\mathrm{H} 1 \mathrm{~A}$ & 0.9800 & $\mathrm{C} 12-\mathrm{H} 12 \mathrm{~A}$ & 0.9800 \\
\hline $\mathrm{C} 1-\mathrm{H} 1 \mathrm{~B}$ & 0.9800 & $\mathrm{C} 12-\mathrm{H} 12 \mathrm{~B}$ & 0.9800 \\
\hline $\mathrm{C} 1-\mathrm{H} 1 \mathrm{C}$ & 0.9800 & $\mathrm{C} 12-\mathrm{H} 12 \mathrm{C}$ & 0.9800 \\
\hline $\mathrm{C} 1-\mathrm{C} 2$ & $1.477(5)$ & & \\
\hline $\mathrm{Mo2}-\mathrm{Mo} 1-\mathrm{Mo} 2$ & $89.98(2)$ & $\mathrm{Cl} 3^{\mathrm{i}}-\mathrm{Mo} 3-\mathrm{Cl} 5$ & $89.98(3)$ \\
\hline $\mathrm{Mo}^{\mathrm{i}}-\mathrm{Mo} 1-\mathrm{Mo}^{\mathrm{i}}$ & $60.005(19)$ & $\mathrm{Cl} 3^{\mathrm{i}}-\mathrm{Mo} 3-\mathrm{Cl} 6$ & $175.12(3)$ \\
\hline Mo2 - -Mo1-Mo3 & $60.202(14)$ & $\mathrm{Cl} 5-\mathrm{Mo} 3-\mathrm{Mo} 1$ & $58.18(2)$ \\
\hline Mo3 ${ }^{\mathrm{i}}-\mathrm{Mo1}-\mathrm{Mo} 2$ & $59.977(16)$ & $\mathrm{Cl} 5-\mathrm{Mo} 3-\mathrm{Mol}^{\mathrm{i}}$ & $118.35(2)$ \\
\hline Mo3-Mo1-Mo2 & $59.763(15)$ & $\mathrm{Cl} 5-\mathrm{Mo} 3-\mathrm{Mo}^{\mathrm{i}}$ & $58.17(2)$ \\
\hline Mo3 ${ }^{\mathrm{i}}-\mathrm{Mo1}-\mathrm{Mo3}$ & $89.98(2)$ & $\mathrm{Cl} 5-\mathrm{Mo} 3-\mathrm{Mo} 2$ & $118.46(3)$ \\
\hline $\mathrm{Cl} 2-\mathrm{Mo} 1-\mathrm{Mo} 2$ & $118.05(2)$ & $\mathrm{Cl} 6-\mathrm{Mo} 3-\mathrm{Mo} 1$ & $58.25(2)$ \\
\hline $\mathrm{Cl} 2-\mathrm{Mo} 1-\mathrm{Mo} 2^{\mathrm{i}}$ & $58.24(2)$ & $\mathrm{Cl} 6-\mathrm{Mo} 3-\mathrm{Mol}^{\mathrm{i}}$ & $117.84(3)$ \\
\hline $\mathrm{Cl} 2-\mathrm{Mo} 1-\mathrm{Mo}^{\mathrm{i}}$ & $58.09(2)$ & $\mathrm{Cl} 6-\mathrm{Mo} 3-\mathrm{Mo} 2$ & $58.02(2)$ \\
\hline $\mathrm{Cl} 2-\mathrm{Mo} 1-\mathrm{Mo} 3$ & $118.42(3)$ & $\mathrm{Cl} 6-\mathrm{Mo} 3-\mathrm{Mo}^{\mathrm{i}}$ & $117.94(2)$ \\
\hline $\mathrm{Cl} 2-\mathrm{Mo} 1-\mathrm{Cl} 5$ & $90.29(3)$ & $\mathrm{Cl} 6-\mathrm{Mo} 3-\mathrm{Cl} 5$ & $90.12(3)$ \\
\hline $\mathrm{Cl} 2-\mathrm{Mo} 1-\mathrm{Cl} 6$ & $175.36(2)$ & $\mathrm{Cl} 7-\mathrm{Mo} 3-\mathrm{Mo} 1$ & $134.62(3)$ \\
\hline $\mathrm{Cl} 3-\mathrm{Mo} 1-\mathrm{Mo} 2$ & $58.22(2)$ & $\mathrm{Cl} 7-\mathrm{Mo} 3-\mathrm{Mol}^{\mathrm{i}}$ & $135.36(2)$ \\
\hline $\mathrm{Cl} 3-\mathrm{Mo} 1-\mathrm{Mo} 2^{\mathrm{i}}$ & $118.22(2)$ & $\mathrm{Cl} 7-\mathrm{Mo} 3-\mathrm{Mo}^{\mathrm{i}}$ & $134.89(3)$ \\
\hline $\mathrm{Cl} 3-\mathrm{Mo} 1-\mathrm{Mo}^{\mathrm{i}}$ & $58.23(2)$ & $\mathrm{Cl} 7-\mathrm{Mo} 3-\mathrm{Mo} 2$ & $135.11(3)$ \\
\hline $\mathrm{Cl} 3-\mathrm{Mo} 1-\mathrm{Mo} 3$ & $117.98(2)$ & $\mathrm{Cl} 7-\mathrm{Mo} 3-\mathrm{Cl}^{\mathrm{i}}$ & $92.39(3)$ \\
\hline $\mathrm{Cl} 3-\mathrm{Mo} 1-\mathrm{Cl} 2$ & $89.80(3)$ & $\mathrm{Cl} 7-\mathrm{Mo} 3-\mathrm{Cl}^{3}{ }^{\mathrm{i}}$ & $92.59(3)$ \\
\hline $\mathrm{Cl} 3-\mathrm{Mo} 1-\mathrm{Cl} 5$ & $175.65(2)$ & $\mathrm{Cl} 7-\mathrm{Mo} 3-\mathrm{Cl} 5$ & $91.72(3)$ \\
\hline $\mathrm{Cl} 3-\mathrm{Mo} 1-\mathrm{Cl} 6$ & $89.39(3)$ & $\mathrm{Cl} 7-\mathrm{Mo} 3-\mathrm{Cl} 6$ & $92.28(3)$ \\
\hline $\mathrm{Cl} 4-\mathrm{Mo} 1-\mathrm{Mo} 2^{\mathrm{i}}$ & $134.02(3)$ & $\mathrm{Mo} 2 \mathrm{i}-\mathrm{Cl} 2-\mathrm{Mo} 1$ & $63.41(2)$ \\
\hline $\mathrm{Cl} 4-\mathrm{Mo} 1-\mathrm{Mo} 2$ & $136.00(2)$ & $\mathrm{Mo}^{\mathrm{i}}-\mathrm{Cl} 2-\mathrm{Mo} 1$ & $63.67(2)$ \\
\hline $\mathrm{Cl} 4-\mathrm{Mo} 1-\mathrm{Mo}^{\mathrm{i}}$ & $135.46(3)$ & $\mathrm{Mo} 3-\mathrm{Cl} 2-\mathrm{Mo} 2^{\mathrm{i}}$ & $63.59(2)$ \\
\hline $\mathrm{Cl} 14-\mathrm{Mo} 1-\mathrm{Mo} 3$ & $134.55(2)$ & $\mathrm{Mo} 1-\mathrm{Cl} 3-\mathrm{Mo} 2$ & $63.92(2)$ \\
\hline $\mathrm{Cl} 4-\mathrm{Mo} 1-\mathrm{Cl} 2$ & $91.90(3)$ & $\mathrm{Mo} 1-\mathrm{Cl} 3-\mathrm{Mo}^{\mathrm{i}}$ & $63.73(2)$ \\
\hline $\mathrm{Cl} 4-\mathrm{Mo} 1-\mathrm{Cl} 3$ & $93.21(3)$ & $\mathrm{Mo} 3-\mathrm{Cl} 3-\mathrm{Mo} 2$ & $63.69(2)$ \\
\hline $\mathrm{Cl} 4-\mathrm{Mo} 1-\mathrm{Cl} 5$ & $91.13(3)$ & $\mathrm{Mo} 1-\mathrm{Cl} 5-\mathrm{Mo}^{\mathrm{i}}$ & $63.26(2)$ \\
\hline $\mathrm{Cl} 4-\mathrm{Mo} 1-\mathrm{Cl} 6$ & $92.71(3)$ & $\mathrm{Mo} 1-\mathrm{Cl} 5-\mathrm{Mo} 3$ & $63.48(2)$ \\
\hline $\mathrm{Cl} 5-\mathrm{Mo} 1-\mathrm{Mo}^{\mathrm{i}}$ & $58.39(2)$ & $\mathrm{Mo} 2 \mathrm{i}-\mathrm{Cl} 5-\mathrm{Mo} 3$ & $63.55(2)$ \\
\hline $\mathrm{Cl} 5-\mathrm{Mo} 1-\mathrm{Mo} 2$ & $118.08(2)$ & $\mathrm{Mo} 2-\mathrm{Cl} 6-\mathrm{Mo} 1$ & $63.94(2)$ \\
\hline $\mathrm{Cl} 15-\mathrm{Mo} 1-\mathrm{Mo} 3$ & $58.33(2)$ & $\mathrm{Mo} 2-\mathrm{Cl} 6-\mathrm{Mo} 3$ & $63.58(3)$ \\
\hline $\mathrm{Cl} 5-\mathrm{Mo} 1-\mathrm{Mo}^{\mathrm{i}}$ & $118.37(2)$ & $\mathrm{Mo} 3-\mathrm{Cl} 6-\mathrm{Mo} 1$ & $63.56(2)$ \\
\hline $\mathrm{Cl} 5-\mathrm{Mo1}-\mathrm{Cl} 6$ & $90.17(3)$ & $\mathrm{C} 2-\mathrm{N} 1-\mathrm{H} 1$ & $117(2)$ \\
\hline $\mathrm{Cl} 6-\mathrm{Mo} 1-\mathrm{Mo}^{\mathrm{i}}$ & $118.37(2)$ & $\mathrm{C} 2-\mathrm{N} 1-\mathrm{C} 5$ & $129.1(3)$ \\
\hline $\mathrm{Cl} 16-\mathrm{Mo} 1-\mathrm{Mo} 2$ & $57.82(2)$ & $\mathrm{C} 5-\mathrm{N} 1-\mathrm{H} 1$ & $114(2)$ \\
\hline $\mathrm{Cl} 16-\mathrm{Mo} 1-\mathrm{Mo} 3$ & $58.19(2)$ & $\mathrm{H} 1 \mathrm{~A}-\mathrm{C} 1-\mathrm{H} 1 \mathrm{~B}$ & 109.5 \\
\hline $\mathrm{Cl} 6-\mathrm{Mo} 1-\mathrm{Mo}^{\mathrm{i}}$ & $117.79(2)$ & $\mathrm{H} 1 \mathrm{~A}-\mathrm{C} 1-\mathrm{H} 1 \mathrm{C}$ & 109.5 \\
\hline Mo1 ${ }^{\mathrm{i}}-\mathrm{Mo2}-\mathrm{Mo1}$ & $90.02(2)$ & $\mathrm{H} 1 \mathrm{~B}-\mathrm{C} 1-\mathrm{H} 1 \mathrm{C}$ & 109.5 \\
\hline $\mathrm{Mo1}{ }^{\mathrm{i}}-\mathrm{Mo} 2-\mathrm{Mo} 3$ & $60.165(17)$ & $\mathrm{C} 2-\mathrm{C} 1-\mathrm{H} 1 \mathrm{~A}$ & 109.5 \\
\hline $\mathrm{Mo}^{\mathrm{i}}-\mathrm{Mo} 2-\mathrm{Mo}^{\mathrm{i}}$ & $60.081(15)$ & $\mathrm{C} 2-\mathrm{C} 1-\mathrm{H} 1 \mathrm{~B}$ & 109.5 \\
\hline Mo3-Mo2-Mo1 & $59.951(17)$ & $\mathrm{C} 2-\mathrm{C} 1-\mathrm{H} 1 \mathrm{C}$ & 109.5 \\
\hline
\end{tabular}




$$
\begin{aligned}
& \text { Mo3 - Mo2-Mo1 } \\
& \text { Mo3-Mo2- } \mathrm{Mo}^{\mathrm{i}} \\
& \text { Cl1-Mo2-Mo1 } \\
& \text { Cl1-Mo2-Mo1 }{ }^{\mathrm{i}} \\
& \text { Cl1-Mo2-Mo3 } \\
& \text { Cl1-Mo2- } \mathrm{Mo}^{\mathrm{i}} \\
& \mathrm{Cl1}-\mathrm{Mo} 2-\mathrm{Cl}_{2}^{\mathrm{i}} \\
& \mathrm{Cl} 1-\mathrm{Mo} 2-\mathrm{Cl} 3 \\
& \mathrm{Cl} 1-\mathrm{Mo} 2-\mathrm{Cl}^{\mathrm{i}} \\
& \mathrm{Cl} 1-\mathrm{Mo} 2-\mathrm{Cl} 6 \\
& \mathrm{Cl} 2 \mathrm{i}-\mathrm{Mo} 2-\mathrm{Mo}^{\mathrm{i}} \\
& \mathrm{Cl} 2 \mathrm{i}-\mathrm{Mo} 2-\mathrm{Mo} 1 \\
& \mathrm{Cl} 2{ }^{\mathrm{i}}-\mathrm{Mo} 2-\mathrm{Mo}^{\mathrm{i}} \\
& \mathrm{C} 12-\mathrm{Mo} 2-\mathrm{Mo} 3 \\
& \mathrm{Cl} 2-\mathrm{Mo} 2-\mathrm{Cl} 3 \\
& \mathrm{Cl} 2^{\mathrm{i}}-\mathrm{Mo} 2-\mathrm{Cl}^{\mathrm{i}} \\
& \mathrm{Cl} 3-\mathrm{Mo} 2-\mathrm{Mo} 1 \\
& \mathrm{Cl} 3-\mathrm{Mo} 2-\mathrm{Mo}^{\mathrm{i}} \\
& \mathrm{Cl} 3-\mathrm{Mo} 2-\mathrm{Mo}^{\mathrm{i}} \\
& \mathrm{Cl} 3-\mathrm{Mo} 2-\mathrm{Mo} 3 \\
& \mathrm{Cl} 3-\mathrm{Mo} 2-\mathrm{Cl}^{\mathrm{i}} \\
& \mathrm{C} 15^{\mathrm{i}}-\mathrm{Mo} 2-\mathrm{Mo} 1 \\
& \mathrm{Cl} 5^{\mathrm{i}}-\mathrm{Mo} 2-\mathrm{Mo}^{\mathrm{i}} \\
& \mathrm{C} 15^{\mathrm{i}}-\mathrm{Mo} 2-\mathrm{Mo}^{\mathrm{i}} \\
& \mathrm{Cl} 1 \text { - }-\mathrm{Mo} 2-\mathrm{Mo} 3 \\
& \text { Cl6-Mo2-Mo1 } \\
& \text { Cl6-Mo2-Mo1 }{ }^{\mathrm{i}} \\
& \text { Cl6-Mo2-Mo3 }{ }^{\mathrm{i}} \\
& \text { Cl6-Mo2-Mo3 } \\
& \mathrm{Cl} 6-\mathrm{Mo} 2-\mathrm{Cl}_{2} \mathrm{i} \\
& \mathrm{Cl} 6-\mathrm{Mo} 2-\mathrm{Cl} 3 \\
& \mathrm{Cl} 6-\mathrm{Mo} 2-\mathrm{Cl}^{\mathrm{i}} \\
& \text { Mo1 }-\mathrm{Mo3}-\mathrm{Mo1} \\
& \mathrm{Mo1}-\mathrm{Mo3}-\mathrm{Mo2}^{\mathrm{i}} \\
& \mathrm{Mo1}{ }^{\mathrm{i}}-\mathrm{Mo3}-\mathrm{Mo}^{\mathrm{i}} \\
& \text { Mo2-Mo3-Mo1 } \\
& \text { Mo2-Mo3-Mo1 }{ }^{\mathrm{i}} \\
& \mathrm{Mo2}-\mathrm{Mo3}-\mathrm{Mo}^{\mathrm{i}} \\
& \mathrm{Cl} 2 \text { - }-\mathrm{Mo} 3-\mathrm{Mo} 1 \\
& \mathrm{Cl} 2^{\mathrm{i}}-\mathrm{Mo} 3-\mathrm{Mo}^{\mathrm{i}} \\
& \mathrm{Cl} 2-\mathrm{Mo} 3-\mathrm{Mo} 2 \\
& \mathrm{Cl} 2 \mathrm{i}-\mathrm{Mo} 3-\mathrm{Mo}^{2} \\
& \mathrm{Cl} 2^{\mathrm{i}}-\mathrm{Mo} 3-\mathrm{Cl}^{\mathrm{i}} \\
& \mathrm{Cl} 2-\mathrm{Mo} 3-\mathrm{Cl} 5 \\
& \mathrm{Cl} 2-\mathrm{Mo} 3-\mathrm{Cl} 6 \\
& \mathrm{Cl} 3^{\mathrm{i}}-\mathrm{Mo} 3-\mathrm{Mol}^{\mathrm{i}} \\
& \mathrm{Cl}^{2}-\mathrm{Mo} 3-\mathrm{Mo} 1 \\
& \mathrm{Cl} 3{ }^{\mathrm{i}}-\mathrm{Mo} 3-\mathrm{Mo}^{2}
\end{aligned}
$$

$59.830(17)$

\begin{tabular}{|c|c|}
\hline $\mathrm{N} 1-\mathrm{C} 2-\mathrm{C} 1$ & $118.1(3)$ \\
\hline $\mathrm{N} 1-\mathrm{C} 2-\mathrm{C} 3$ & $122.8(3)$ \\
\hline $\mathrm{C} 1-\mathrm{C} 2-\mathrm{C} 3$ & $119.1(3)$ \\
\hline $\mathrm{C} 2-\mathrm{C} 3-\mathrm{H} 3 \mathrm{~A}$ & 109.5 \\
\hline $\mathrm{C} 2-\mathrm{C} 3-\mathrm{H} 3 \mathrm{~B}$ & 109.5 \\
\hline $\mathrm{C} 2-\mathrm{C} 3-\mathrm{H} 3 \mathrm{C}$ & 109.5 \\
\hline $\mathrm{H} 3 \mathrm{~A}-\mathrm{C} 3-\mathrm{H} 3 \mathrm{~B}$ & 109.5 \\
\hline $\mathrm{H} 3 \mathrm{~A}-\mathrm{C} 3-\mathrm{H} 3 \mathrm{C}$ & 109.5 \\
\hline $\mathrm{H} 3 \mathrm{~B}-\mathrm{C} 3-\mathrm{H} 3 \mathrm{C}$ & 109.5 \\
\hline $\mathrm{C} 5-\mathrm{C} 4-\mathrm{H} 4$ & 120.4 \\
\hline $\mathrm{C} 6^{\mathrm{ii}}-\mathrm{C} 4-\mathrm{H} 4$ & 120.4 \\
\hline $\mathrm{C} 6^{\mathrm{ii}}-\mathrm{C} 4-\mathrm{C} 5$ & $119.1(3)$ \\
\hline $\mathrm{C} 4-\mathrm{C} 5-\mathrm{N} 1$ & $121.0(3)$ \\
\hline $\mathrm{C} 4-\mathrm{C} 5-\mathrm{C} 6$ & $121.2(3)$ \\
\hline $\mathrm{C} 6-\mathrm{C} 5-\mathrm{N} 1$ & $117.7(3)$ \\
\hline $\mathrm{C} 4 \mathrm{ii}-\mathrm{C} 6-\mathrm{C} 5$ & $119.6(3)$ \\
\hline $\mathrm{C} 4{ }^{\mathrm{ii}}-\mathrm{C} 6-\mathrm{H} 6$ & 120.2 \\
\hline $\mathrm{C} 5-\mathrm{C} 6-\mathrm{H} 6$ & 120.2 \\
\hline $\mathrm{H} 7 \mathrm{~A}-\mathrm{C} 7-\mathrm{H} 7 \mathrm{~B}$ & 109.5 \\
\hline $\mathrm{H} 7 \mathrm{~A}-\mathrm{C} 7-\mathrm{H} 7 \mathrm{C}$ & 109.5 \\
\hline $\mathrm{H} 7 \mathrm{~B}-\mathrm{C} 7-\mathrm{H} 7 \mathrm{C}$ & 109.5 \\
\hline $\mathrm{C} 8-\mathrm{C} 7-\mathrm{H} 7 \mathrm{~A}$ & 109.5 \\
\hline $\mathrm{C} 8-\mathrm{C} 7-\mathrm{H} 7 \mathrm{~B}$ & 109.5 \\
\hline $\mathrm{C} 8-\mathrm{C} 7-\mathrm{H} 7 \mathrm{C}$ & 109.5 \\
\hline $\mathrm{O} 1-\mathrm{C} 8-\mathrm{C} 7$ & $121.9(4)$ \\
\hline $\mathrm{O} 1-\mathrm{C} 8-\mathrm{C} 9$ & $120.5(3)$ \\
\hline $\mathrm{C} 7-\mathrm{C} 8-\mathrm{C} 9$ & $117.7(3)$ \\
\hline $\mathrm{C} 8-\mathrm{C} 9-\mathrm{H} 9 \mathrm{~A}$ & 109.5 \\
\hline $\mathrm{C} 8-\mathrm{C} 9-\mathrm{H} 9 \mathrm{~B}$ & 109.5 \\
\hline $\mathrm{C} 8-\mathrm{C} 9-\mathrm{H} 9 \mathrm{C}$ & 109.5 \\
\hline $\mathrm{H} 9 \mathrm{~A}-\mathrm{C} 9-\mathrm{H} 9 \mathrm{~B}$ & 109.5 \\
\hline $\mathrm{H} 9 \mathrm{~A}-\mathrm{C} 9-\mathrm{H} 9 \mathrm{C}$ & 109.5 \\
\hline $\mathrm{H} 9 \mathrm{~B}-\mathrm{C} 9-\mathrm{H} 9 \mathrm{C}$ & 109.5 \\
\hline $\mathrm{O} 2-\mathrm{C} 10-\mathrm{C} 11$ & $122.1(9)$ \\
\hline $\mathrm{O} 2-\mathrm{C} 10-\mathrm{C} 12$ & $120.8(9)$ \\
\hline $\mathrm{C} 12-\mathrm{C} 10-\mathrm{C} 11$ & $116.9(14)$ \\
\hline $\mathrm{C} 10-\mathrm{C} 11-\mathrm{H} 11 \mathrm{~A}$ & 109.5 \\
\hline $\mathrm{C} 10-\mathrm{C} 11-\mathrm{H} 11 \mathrm{~B}$ & 109.5 \\
\hline $\mathrm{C} 10-\mathrm{C} 11-\mathrm{H} 11 \mathrm{C}$ & 109.5 \\
\hline $\mathrm{H} 11 \mathrm{~A}-\mathrm{C} 11-\mathrm{H} 11 \mathrm{~B}$ & 109.5 \\
\hline $\mathrm{H} 11 \mathrm{~A}-\mathrm{C} 11-\mathrm{H} 11 \mathrm{C}$ & 109.5 \\
\hline $\mathrm{H} 11 \mathrm{~B}-\mathrm{C} 11-\mathrm{H} 11 \mathrm{C}$ & 109.5 \\
\hline $\mathrm{C} 10-\mathrm{C} 12-\mathrm{H} 12 \mathrm{~A}$ & 109.5 \\
\hline $\mathrm{C} 10-\mathrm{C} 12-\mathrm{H} 12 \mathrm{~B}$ & 109.5 \\
\hline $\mathrm{C} 10-\mathrm{C} 12-\mathrm{H} 12 \mathrm{C}$ & 109.5 \\
\hline $\mathrm{H} 12 \mathrm{~A}-\mathrm{C} 12-\mathrm{H} 12 \mathrm{~B}$ & 109.5 \\
\hline $\mathrm{H} 12 \mathrm{~A}-\mathrm{C} 12-\mathrm{H} 12 \mathrm{C}$ & 109.5 \\
\hline $\mathrm{H} 12 \mathrm{~B}-\mathrm{C} 12-\mathrm{H} 12 \mathrm{C}$ & 109.5 \\
\hline
\end{tabular}

$90.00(2)$

135.83 (3)

$134.15(2)$

$134.88(3)$

$135.11(2)$

$91.56(3)$

$93.08(3)$

$91.61(3)$

$92.55(3)$

$58.35(2)$

$118.11(2)$

118.40 (2)

$58.18(2)$

175.35 (2)

$90.33(3)$

$57.85(2)$

$118.13(2)$

58.07 (2)

$117.79(2)$

$89.93(3)$

118.09 (2)

$58.35(2)$

58.28 (2)

118.49 (2)

$58.24(2)$

$118.54(3)$

118.06 (2)

$58.39(2)$

89.98 (3)

$89.42(3)$

$175.82(2)$

$90.025(19)$

$59.719(18)$

$60.193(13)$

60.287 (13)

$59.830(12)$

$90.00(2)$

118.50 (2)

$58.24(2)$

$58.23(2)$

$118.42(2)$

89.77 (3)

$175.88(3)$

89.79 (3)

58.03 (2)

117.94 (2)

58.24 (2)
$118.1(3)$
$122.8(3)$
$119.1(3)$
109.5
109.5
109.5
109.5
109.5
109.5
120.4
120.4
$119.1(3)$
$121.0(3)$
$121.2(3)$
$117.7(3)$
$119.6(3)$
120.2
120.2
109.5
109.5
109.5
109.5
109.5
109.5
$121.9(4)$
$120.5(3)$
$117.7(3)$
109.5
109.5
109.5
109.5
109.5
109.5
$122.1(9)$
$120.8(9)$
$116.9(14)$
109.5
109.5
109.5
109.5
109.5
109.5
109.5
109.5
109.5
109.5
109.5
109.5
1 
$\mathrm{Cl} 3{ }^{\mathrm{i}}-\mathrm{Mo} 3-\mathrm{Mo} 2$

$\mathrm{N} 1-\mathrm{C} 5-\mathrm{C} 6-\mathrm{C} 4{ }^{\mathrm{ii}}$

$\mathrm{C} 2-\mathrm{N} 1-\mathrm{C} 5-\mathrm{C} 4$

$\mathrm{C} 2-\mathrm{N} 1-\mathrm{C} 5-\mathrm{C} 6$

$\mathrm{C} 4-\mathrm{C} 5-\mathrm{C} 6-\mathrm{C}^{\mathrm{ii}}$
$117.85(2)$

$176.9(3)$

$-46.6(5)$

$136.5(3)$

$-0.1(5)$
$\mathrm{C} 5-\mathrm{N} 1-\mathrm{C} 2-\mathrm{C} 1$

$\mathrm{C} 5-\mathrm{N} 1-\mathrm{C} 2-\mathrm{C} 3$

$\mathrm{C} 6$ ii- $44-\mathrm{C} 5-\mathrm{N} 1$

$\mathrm{C} 6$ ii- $4-\mathrm{C} 5-\mathrm{C} 6$
$174.4(3)$

$-4.4(5)$

$-176.7(3)$

$0.1(5)$

Symmetry codes: (i) $-x+1,-y+1,-z+1$; (ii) $-x,-y,-z+1$.

Hydrogen-bond geometry $\left(\AA,{ }^{\circ}\right)$

\begin{tabular}{lllll}
\hline$D-\mathrm{H} \cdots A$ & $D-\mathrm{H}$ & $\mathrm{H} \cdots A$ & $D \cdots A$ & $D-\mathrm{H} \cdots A$ \\
\hline $\mathrm{N} 1-\mathrm{H} 1 \cdots \mathrm{O} 1^{\mathrm{iii}}$ & $0.87(2)$ & $1.93(2)$ & $2.791(4)$ & $172(3)$ \\
\hline
\end{tabular}

Symmetry code: (iii) $x, y-1, z$.

1,1'-Dimethyl-4,4'-bipyridinium octa- $\mu_{3}$-chlorido-hexachlorido-octahedro-hexamolybdate $N, N$ -

dimethylformamide tetrasolvate (4)

\section{Crystal data}

$\left(\mathrm{C}_{12} \mathrm{H}_{14} \mathrm{~N}_{2}\right)\left[\mathrm{Mo}_{6} \mathrm{Cl}_{8} \mathrm{Cl}_{6}\right] \cdot 4 \mathrm{C}_{3} \mathrm{H}_{7} \mathrm{NO}$

$M_{r}=1550.57$

Triclinic, $P \overline{1}$

$a=9.8252(11) \AA$

$b=10.0933(11) \AA$

$c=12.6319(15) \AA$

$\alpha=107.395(3)^{\circ}$

$\beta=91.881(3)^{\circ}$

$\gamma=93.309(3)^{\circ}$

$V=1191.8(2) \AA^{3}$

\section{Data collection}

Bruker SMART X2S benchtop diffractometer

Radiation source: sealed microfocus source, XOS X-beam microfocus source

Graphite monochromator

Detector resolution: 8.3330 pixels $\mathrm{mm}^{-1}$

$\varphi$ and $\omega$ scans

Absorption correction: multi-scan

(SADABS; Bruker, 2012)

\section{Refinement}

Refinement on $F^{2}$

Least-squares matrix: full

$R\left[F^{2}>2 \sigma\left(F^{2}\right)\right]=0.020$

$w R\left(F^{2}\right)=0.049$

$S=1.02$

4187 reflections

250 parameters

0 restraints

Primary atom site location: heavy-atom method

Hydrogen site location: inferred from

neighbouring sites
$Z=1$

$F(000)=750$

$D_{\mathrm{x}}=2.161 \mathrm{Mg} \mathrm{m}^{-3}$

Mo $K \alpha$ radiation, $\lambda=0.71073 \AA$

Cell parameters from 6604 reflections

$\theta=2.6-25.0^{\circ}$

$\mu=2.35 \mathrm{~mm}^{-1}$

$T=200 \mathrm{~K}$

Block, orange

$0.32 \times 0.30 \times 0.28 \mathrm{~mm}$

$T_{\text {min }}=0.815, T_{\max }=1.000$

11498 measured reflections

4187 independent reflections

3743 reflections with $I>2 \sigma(I)$

$R_{\text {int }}=0.024$

$\theta_{\max }=25.1^{\circ}, \theta_{\min }=2.3^{\circ}$

$h=-11 \rightarrow 11$

$k=-11 \rightarrow 11$

$l=-15 \rightarrow 15$

$\mathrm{H}$-atom parameters constrained

$w=1 /\left[\sigma^{2}\left(F_{\mathrm{o}}^{2}\right)+(0.0184 P)^{2}+0.8263 P\right]$ where $P=\left(F_{\mathrm{o}}^{2}+2 F_{\mathrm{c}}^{2}\right) / 3$

$(\Delta / \sigma)_{\max }=0.002$

$\Delta \rho_{\max }=0.42$ e $\AA^{-3}$

$\Delta \rho_{\text {min }}=-0.44$ e $\AA^{-3}$

Extinction correction: SHELXL2018 (Sheldrick, 2015),

$\mathrm{Fc}^{*}=\mathrm{kFc}\left[1+0.001 \mathrm{xFc}^{2} \lambda^{3} / \sin (2 \theta)\right]^{-1 / 4}$

Extinction coefficient: 0.0075 (3) 


\section{Special details}

Geometry. All esds (except the esd in the dihedral angle between two 1.s. planes) are estimated using the full covariance matrix. The cell esds are taken into account individually in the estimation of esds in distances, angles and torsion angles; correlations between esds in cell parameters are only used when they are defined by crystal symmetry. An approximate (isotropic) treatment of cell esds is used for estimating esds involving l.s. planes.

Fractional atomic coordinates and isotropic or equivalent isotropic displacement parameters $\left(\AA^{2}\right)$

\begin{tabular}{|c|c|c|c|c|}
\hline & $x$ & $y$ & $z$ & $U_{\text {iso }} * / U_{\text {eq }}$ \\
\hline Mo1 & $0.34350(2)$ & $0.52105(2)$ & $0.42443(2)$ & $0.01878(7)$ \\
\hline Mo2 & $0.43880(2)$ & $0.61443(2)$ & $0.62913(2)$ & $0.01930(7)$ \\
\hline Mo3 & $0.41622(2)$ & $0.34763(2)$ & $0.52876(2)$ & $0.01916(7)$ \\
\hline $\mathrm{Cl} 4$ & $0.21336(6)$ & $0.48336(6)$ & $0.57792(5)$ & $0.02520(14)$ \\
\hline $\mathrm{Cl} 2$ & $0.51113(6)$ & $0.44527(7)$ & $0.72253(5)$ & $0.02551(14)$ \\
\hline $\mathrm{Cl} 7$ & $0.30745(7)$ & $0.14843(7)$ & $0.56719(6)$ & $0.03486(17)$ \\
\hline $\mathrm{Cl} 6$ & $0.35663(7)$ & $0.76331(7)$ & $0.79813(6)$ & $0.03874(18)$ \\
\hline $\mathrm{Cl} 3$ & $0.32970(6)$ & $0.26780(6)$ & $0.33243(5)$ & $0.02516(14)$ \\
\hline Cl1 & $0.37330(6)$ & $0.77313(6)$ & $0.52328(5)$ & $0.02510(14)$ \\
\hline $\mathrm{Cl} 5$ & $0.13401(6)$ & $0.54800(7)$ & $0.32618(5)$ & $0.03256(16)$ \\
\hline N1 & $0.0168(2)$ & $0.9093(3)$ & $0.7467(2)$ & $0.0392(6)$ \\
\hline $\mathrm{C} 3$ & $0.0031(2)$ & $0.9806(3)$ & $0.5519(2)$ & $0.0299(6)$ \\
\hline C6 & 0.0223 (4) & $0.8700(4)$ & $0.8508(3)$ & $0.0560(9)$ \\
\hline H6A & 0.092144 & 0.930439 & 0.903109 & $0.084^{*}$ \\
\hline H6B & 0.045141 & 0.772867 & 0.834244 & $0.084^{*}$ \\
\hline $\mathrm{H} 6 \mathrm{C}$ & -0.066769 & 0.880619 & 0.883944 & $0.084^{*}$ \\
\hline $\mathrm{O} 1$ & 0.0987 (4) & $0.2529(3)$ & 0.9699 (2) & 0.0947 (11) \\
\hline N2 & $0.1930(3)$ & $0.4599(3)$ & $0.9693(2)$ & $0.0462(7)$ \\
\hline $\mathrm{C} 7$ & $0.1610(5)$ & $0.3616(4)$ & $1.0150(3)$ & $0.0661(11)$ \\
\hline H7 & 0.190127 & 0.378592 & 1.090665 & $0.079^{*}$ \\
\hline C9 & 0.1539 (4) & 0.4414 (4) & $0.8553(3)$ & $0.0642(10)$ \\
\hline H9A & 0.191569 & 0.357250 & 0.808268 & $0.096^{*}$ \\
\hline H9B & 0.189323 & 0.522299 & 0.834281 & $0.096^{*}$ \\
\hline H9C & 0.054111 & 0.432088 & 0.845257 & $0.096^{*}$ \\
\hline $\mathrm{C} 8$ & $0.2613(6)$ & $0.5918(5)$ & $1.0316(4)$ & 0.0954 (17) \\
\hline H8A & 0.274154 & 0.595293 & 1.109641 & $0.143^{*}$ \\
\hline H8B & 0.205703 & 0.667344 & 1.026178 & $0.143^{*}$ \\
\hline $\mathrm{H} 8 \mathrm{C}$ & 0.350353 & 0.602489 & 1.001246 & $0.143^{*}$ \\
\hline $\mathrm{O} 2$ & $0.2430(3)$ & $0.9764(3)$ & $0.1097(2)$ & $0.0629(7)$ \\
\hline N3 & 0.4369 (3) & 0.9015 (3) & $0.1660(2)$ & $0.0425(6)$ \\
\hline $\mathrm{C} 10$ & $0.3573(4)$ & $0.9392(3)$ & $0.0942(3)$ & $0.0479(8)$ \\
\hline H10 & 0.393572 & 0.936671 & 0.024855 & $0.058^{*}$ \\
\hline $\mathrm{C} 12$ & $0.3883(4)$ & 0.9057 (4) & $0.2736(3)$ & 0.0567 (9) \\
\hline $\mathrm{H} 12 \mathrm{~A}$ & 0.311583 & 0.965299 & 0.289780 & $0.085^{*}$ \\
\hline H12B & 0.462220 & 0.943321 & 0.330545 & $0.085^{*}$ \\
\hline $\mathrm{H} 12 \mathrm{C}$ & 0.358102 & 0.811373 & 0.273342 & $0.085^{*}$ \\
\hline $\mathrm{C} 11$ & $0.5716(4)$ & $0.8576(4)$ & 0.1409 (4) & $0.0698(11)$ \\
\hline H11A & 0.576876 & 0.762359 & 0.144373 & $0.105^{*}$ \\
\hline H11B & 0.638360 & 0.920002 & 0.195053 & $0.105^{*}$ \\
\hline
\end{tabular}




$\begin{array}{lllll}\mathrm{H} 11 \mathrm{C} & 0.591481 & 0.860583 & 0.066069 & 0.105^{*} \\ \mathrm{C} 2 & 0.0219(3) & 0.8454(3) & 0.5522(2) & 0.0407(7) \\ \mathrm{H} 2 & 0.030656 & 0.774946 & 0.483942 & 0.049^{*} \\ \mathrm{C} 1 & 0.0281(3) & 0.8128(4) & 0.6492(3) & 0.0450(8) \\ \mathrm{H} 1 & 0.040636 & 0.719562 & 0.647548 & 0.054^{*} \\ \mathrm{C} 4 & -0.0086(4) & 1.0768(3) & 0.6542(3) & 0.0499(9) \\ \text { H4 } & -0.021238 & 1.170823 & 0.658585 & 0.060^{*} \\ \mathrm{C} 5 & -0.0023(4) & 1.0392(3) & 0.7493(3) & 0.0539(9) \\ \mathrm{H} 5 & -0.011812 & 1.107404 & 0.818648 & 0.065^{*}\end{array}$

Atomic displacement parameters $\left(\AA^{2}\right)$

\begin{tabular}{lllllll}
\hline & $U^{11}$ & $U^{22}$ & $U^{33}$ & $U^{12}$ & $U^{13}$ & $U^{23}$ \\
\hline Mo1 & $0.01652(11)$ & $0.02000(13)$ & $0.02050(12)$ & $0.00181(8)$ & $0.00057(8)$ & $0.00707(9)$ \\
Mo2 & $0.01846(11)$ & $0.01990(13)$ & $0.01947(12)$ & $0.00228(9)$ & $0.00309(8)$ & $0.00547(9)$ \\
Mo3 & $0.01755(11)$ & $0.01909(13)$ & $0.02210(12)$ & $0.00020(8)$ & $0.00183(8)$ & $0.00827(9)$ \\
C14 & $0.0185(3)$ & $0.0295(3)$ & $0.0290(3)$ & $0.0021(2)$ & $0.0059(2)$ & $0.0103(3)$ \\
C12 & $0.0272(3)$ & $0.0301(4)$ & $0.0223(3)$ & $0.0025(3)$ & $0.0018(2)$ & $0.0124(3)$ \\
C17 & $0.0300(3)$ & $0.0327(4)$ & $0.0474(4)$ & $-0.0075(3)$ & $-0.0014(3)$ & $0.0226(3)$ \\
C16 & $0.0430(4)$ & $0.0374(4)$ & $0.0305(4)$ & $0.0072(3)$ & $0.0131(3)$ & $0.0001(3)$ \\
C13 & $0.0239(3)$ & $0.0227(3)$ & $0.0259(3)$ & $-0.0016(2)$ & $-0.0023(2)$ & $0.0038(3)$ \\
C11 & $0.0240(3)$ & $0.0207(3)$ & $0.0318(3)$ & $0.0047(2)$ & $0.0021(2)$ & $0.0090(3)$ \\
C15 & $0.0254(3)$ & $0.0383(4)$ & $0.0335(4)$ & $0.0070(3)$ & $-0.0058(3)$ & $0.0100(3)$ \\
N1 & $0.0294(12)$ & $0.0537(17)$ & $0.0346(13)$ & $0.0046(11)$ & $0.0096(10)$ & $0.0125(12)$ \\
C3 & $0.0193(12)$ & $0.0339(16)$ & $0.0316(14)$ & $-0.0056(11)$ & $0.0078(11)$ & $0.0031(12)$ \\
C6 & $0.054(2)$ & $0.082(3)$ & $0.0421(19)$ & $0.0242(19)$ & $0.0164(16)$ & $0.0289(19)$ \\
O1 & $0.166(3)$ & $0.0601(18)$ & $0.0529(17)$ & $-0.046(2)$ & $0.0022(19)$ & $0.0192(15)$ \\
N2 & $0.0566(17)$ & $0.0467(16)$ & $0.0351(14)$ & $-0.0092(13)$ & $-0.0047(12)$ & $0.0153(13)$ \\
C7 & $0.105(3)$ & $0.061(3)$ & $0.0329(18)$ & $-0.010(2)$ & $0.0046(19)$ & $0.0184(19)$ \\
C9 & $0.078(3)$ & $0.070(3)$ & $0.047(2)$ & $-0.013(2)$ & $-0.0116(19)$ & $0.028(2)$ \\
C8 & $0.132(4)$ & $0.080(3)$ & $0.067(3)$ & $-0.046(3)$ & $-0.033(3)$ & $0.026(3)$ \\
O2 & $0.0563(16)$ & $0.0770(18)$ & $0.0618(16)$ & $0.0167(14)$ & $-0.0073(13)$ & $0.0295(14)$ \\
N3 & $0.0483(15)$ & $0.0320(14)$ & $0.0497(16)$ & $0.0033(12)$ & $-0.0034(13)$ & $0.0165(12)$ \\
C10 & $0.060(2)$ & $0.0404(19)$ & $0.0432(18)$ & $-0.0019(16)$ & $-0.0034(16)$ & $0.0146(16)$ \\
C12 & $0.078(3)$ & $0.048(2)$ & $0.047(2)$ & $0.0120(18)$ & $-0.0094(18)$ & $0.0183(17)$ \\
C11 & $0.056(2)$ & $0.064(3)$ & $0.100(3)$ & $0.0104(19)$ & $0.009(2)$ & $0.038(2)$ \\
C2 & $0.0383(16)$ & $0.0433(19)$ & $0.0360(16)$ & $0.0171(14)$ & $0.0025(13)$ & $0.0024(14)$ \\
C1 & $0.0423(17)$ & $0.049(2)$ & $0.0455(19)$ & $0.0226(15)$ & $0.0062(14)$ & $0.0125(16)$ \\
C4 & $0.079(2)$ & $0.0295(17)$ & $0.0366(17)$ & $-0.0111(16)$ & $0.0227(16)$ & $0.0043(14)$ \\
C5 & $0.080(3)$ & $0.039(2)$ & $0.0360(18)$ & $-0.0106(17)$ & $0.0218(17)$ & $0.0016(15)$ \\
& & & & & & \\
\hline & & & & & & \\
\end{tabular}

Geometric parameters $\left(\AA,{ }^{\circ}\right)$

\begin{tabular}{llll}
\hline Mo1-Mo2 & $2.6043(4)$ & C6-H6C & 0.9800 \\
Mo1-Mo2 & $2.5948(4)$ & O1-C7 & $1.195(4)$ \\
Mo1-Mo3 & $2.6026(3)$ & N2-C7 & $1.318(4)$ \\
Mo1-Mo3 & $2.5996(4)$ & N2-C9 & $1.432(4)$ \\
Mo1-Cl4 & $2.4671(6)$ & N2-C 8 & $1.442(5)$
\end{tabular}




$$
\begin{aligned}
& \mathrm{Mo1}-\mathrm{Cl} 2^{\mathrm{i}} \\
& \mathrm{Mo1}-\mathrm{Cl3} \\
& \mathrm{Mo1}-\mathrm{Cl1} \\
& \mathrm{Mo1}-\mathrm{Cl} 5 \\
& \text { Mo2-Mo3 } \\
& \text { Mo2-Mo3 } \\
& \text { Mo2-Cl4 } \\
& \text { Mo2-Cl2 } \\
& \text { Mo2-Cl6 } \\
& \text { Mo2-Cl3 } \\
& \text { Mo2-Cl1 } \\
& \text { Mo3-Cl4 } \\
& \text { Mo3-Cl2 } \\
& \text { Mo3-Cl7 } \\
& \text { Mo3-Cl3 } \\
& \text { Mo3-Cl1 } \\
& \text { N1-C6 } \\
& \text { N1-C1 } \\
& \text { N1-C5 } \\
& \text { C3-C3 } \\
& \text { C3-C2 } \\
& \text { C3-C4 } \\
& \text { C6-H6A } \\
& \text { C6-H6B }
\end{aligned}
$$

$\mathrm{Mo2}-\mathrm{Mo1}-\mathrm{Mo}^{\mathrm{i}}$

Mo2-Mo1-Mo3 ${ }^{\mathrm{i}}$

Mo2-Mo1-Mo3

Mo3-Mo1-Mo2 ${ }^{\mathrm{i}}$

$\mathrm{Mo}^{\mathrm{i}}-\mathrm{Mo} 1-\mathrm{Mo}^{\mathrm{i}}$

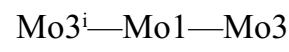

Cl4-Mo1-Mo2 ${ }^{\mathrm{i}}$

C14-Mo1-Mo2

Cl4-Mo1-Mo3 ${ }^{\mathrm{i}}$

Cl4-Mo1-Mo3

$\mathrm{Cl} 4-\mathrm{Mo} 1-\mathrm{Cl}^{\mathrm{i}}$

Cl4-Mo1-Cl1

$\mathrm{C} 12$ - $-\mathrm{Mo} 1-\mathrm{Mo} 2$

$\mathrm{Cl} 2^{\mathrm{i}}-\mathrm{Mo} 1-\mathrm{Mo}^{\mathrm{i}}$

$\mathrm{Cl} 2^{\mathrm{i}}-\mathrm{Mo} 1-\mathrm{Mo}^{\mathrm{i}}$

$\mathrm{C} 12$ - $-\mathrm{Mo} 1-\mathrm{Mo} 3$

$\mathrm{Cl} 2 \mathrm{i}-\mathrm{Mo} 1-\mathrm{Cl} 1$

$\mathrm{Cl} 3-\mathrm{Mo} 1-\mathrm{Mo}^{\mathrm{i}}$

$\mathrm{Cl} 3-\mathrm{Mo} 1-\mathrm{Mo} 2$

$\mathrm{Cl} 3-\mathrm{Mo} 1-\mathrm{Mo} 3$

$\mathrm{Cl} 3-\mathrm{Mo} 1-\mathrm{Mo}^{\mathrm{i}}$

$\mathrm{Cl} 3-\mathrm{Mo1}-\mathrm{Cl} 4$

$\mathrm{Cl} 3-\mathrm{Mo} 1-\mathrm{Cl}_{2}^{\mathrm{i}}$
$2.4692(6)$

$2.4650(7)$

$2.4716(7)$

$2.4392(7)$

2.5949 (3)

2.6037 (4)

$2.4760(6)$

$2.4701(6)$

$2.4088(7)$

$2.4683(6)$

$2.4720(6)$

$2.4745(6)$

$2.4766(7)$

$2.4061(7)$

2.4719 (7)

2.4689 (6)

1.484 (4)

1.334 (4)

1.327 (4)

1.477 (5)

$1.388(4)$

1.377 (4)

0.9800

0.9800

89.921 (11)

$59.942(8)$

$60.128(10)$

59.784 (9)

60.045 (11)

89.992 (11)

118.129 (17)

58.503 (17)

118.428 (17)

58.357 (16)

175.80 (2)

90.24 (2)

118.347 (18)

$58.200(16)$

$58.426(16)$

117.975 (17)

$90.06(2)$

$58.199(15)$

$118.426(17)$

58.314 (17)

118.235 (16)

89.90 (2)

89.50 (2)
C7-H7

C9-H9A

C9-H9B

C9- $\mathrm{H} 9 \mathrm{C}$

$\mathrm{C} 8-\mathrm{H} 8 \mathrm{~A}$

C8-H8B

$\mathrm{C} 8-\mathrm{H} 8 \mathrm{C}$

$\mathrm{O} 2-\mathrm{C} 10$

N3- $\mathrm{C} 10$

N3-C12

N3- 11

C10- 10

C12-H12A

C12-H12B

C12-H12C

C11-H11A

C11-H11B

C11-H11C

$\mathrm{C} 2-\mathrm{H} 2$

$\mathrm{C} 2-\mathrm{C} 1$

$\mathrm{C} 1-\mathrm{H} 1$

$\mathrm{C} 4-\mathrm{H} 4$

$\mathrm{C} 4-\mathrm{C} 5$

$\mathrm{C} 5-\mathrm{H} 5$

$\mathrm{Cl} 7-\mathrm{Mo} 3-\mathrm{Mo}^{\mathrm{i}}$

Cl7-Mo3- $\mathrm{Cl} 4$

$\mathrm{Cl} 7-\mathrm{Mo} 3-\mathrm{Cl} 2$

$\mathrm{Cl} 7-\mathrm{Mo} 3-\mathrm{Cl} 3$

$\mathrm{Cl} 7-\mathrm{Mo} 3-\mathrm{Cl}^{\mathrm{i}}$

$\mathrm{Cl} 3-\mathrm{Mo} 3-\mathrm{Mo} 1$

$\mathrm{Cl} 3-\mathrm{Mo} 3-\mathrm{Mo}^{\mathrm{i}}$

$\mathrm{Cl} 3-\mathrm{Mo} 3-\mathrm{Mo} 2$

$\mathrm{Cl} 3-\mathrm{Mo} 3-\mathrm{Mo}^{\mathrm{i}}$

$\mathrm{Cl} 3-\mathrm{Mo} 3-\mathrm{Cl} 4$

$\mathrm{Cl} 3-\mathrm{Mo} 3-\mathrm{Cl} 2$

Cl1 1 -Mo3-Mo1

Cl1 $-\mathrm{Mo} 3-\mathrm{Mol}^{\mathrm{i}}$

Cl1 $-\mathrm{Mo} 3-\mathrm{Mo}^{\mathrm{i}}$

Cl1 $-\mathrm{Mo} 3-\mathrm{Mo} 2$

$\mathrm{Cl1}$ - $-\mathrm{Mo} 3-\mathrm{Cl} 4$

$\mathrm{Cl1}-\mathrm{Mo} 3-\mathrm{Cl} 2$

$\mathrm{Cl1}-\mathrm{Mo} 3-\mathrm{Cl} 3$

$\mathrm{Mo} 1-\mathrm{Cl} 4-\mathrm{Mo} 2$

$\mathrm{Mo} 1-\mathrm{Cl} 4-\mathrm{Mo} 3$

Mo3-Cl4-Mo2

$\mathrm{Mo1}-\mathrm{Cl} 2-\mathrm{Mo} 2$

$\mathrm{Mo} 1-\mathrm{Cl} 2-\mathrm{Mo} 3$
0.9500

0.9800

0.9800

0.9800

0.9800

0.9800

0.9800

$1.208(4)$

$1.331(4)$

1.445 (4)

$1.435(4)$

0.9500

0.9800

0.9800

0.9800

0.9800

0.9800

0.9800

0.9500

1.361 (4)

0.9500

0.9500

1.363 (4)

0.9500

135.04 (2)

92.33 (2)

92.13 (2)

$92.61(2)$

91.81 (2)

58.057 (16)

118.159 (16)

$117.829(17)$

58.247 (16)

89.57 (2)

$175.25(2)$

$118.500(16)$

$58.302(17)$

$58.376(16)$

$118.352(17)$

175.86 (2)

89.95 (2)

$90.20(2)$

$63.328(16)$

$63.562(15)$

$63.465(17)$

$63.638(16)$

$63.422(17)$ 


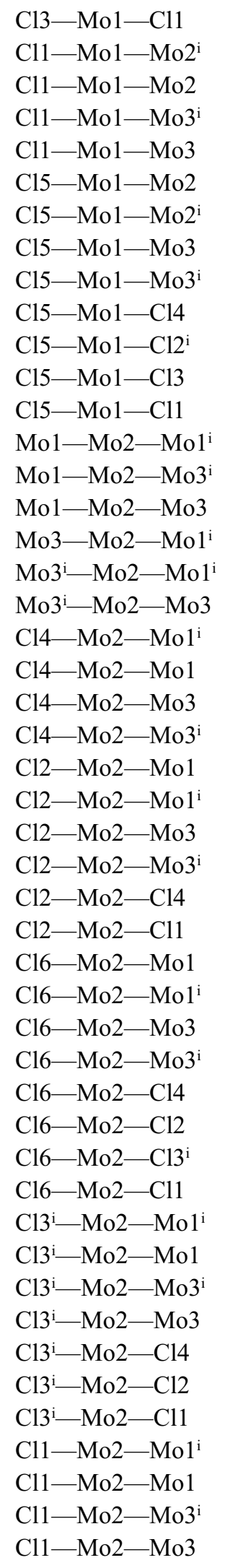

$175.83(2)$
$118.234(17)$
$58.347(15)$
$58.203(15)$
$118.456(18)$
$134.428(19)$
$135.644(19)$
$134.380(19)$
$135.62(2)$
$91.17(2)$
$93.01(2)$
$92.03(2)$
$92.13(2)$
$90.078(10)$
$60.121(11)$
$60.084(8)$
$59.890(9)$
$60.076(9)$
$90.070(10)$
$118.113(18)$
$58.169(15)$
$58.238(16)$
$118.272(17)$
$118.429(18)$
$58.164(15)$
$58.360(17)$
$118.230(17)$
$90.05(2)$
$175.86(2)$
$134.52(2)$
$135.40(2)$
$134.68(2)$
$135.25(2)$
$91.70(2)$
$92.13(2)$
$92.69(2)$
$92.01(3)$
$58.075(17)$
$118.479(16)$
$58.382(16)$
$117.956(16)$
$175.60(2)$
$89.40(2)$
$90.21(2)$
$118.322(16)$
$58.332(17)$
$58.262(16)$
$118.397(19)$

$\begin{array}{ll}\text { Mo2-C12-Mo3 } & 63.519(17) \\ \text { Mo1-C13-Mo2 } & 63.726(16) \\ \text { Mo1-C13-Mo3 } & 63.629(16) \\ \text { Mo2-C13-Mo3 } & 63.372(16) \\ \text { Mo1-C11-Mo2 } & 63.322(17) \\ \text { Mo3-C11-Mo1 } & 63.496(16) \\ \text { Mo3-C11-Mo2 } & 63.363(16) \\ \text { C1-N1-C6 } & 119.9(3) \\ \text { C5-N1-C6 } & 120.5(3) \\ \text { C5-N1-C1 } & 119.6(3) \\ \text { C2-C3-C3 } & 122.0(3) \\ \text { C4-C3-C3 } & 121.9(3) \\ \text { C4-C3-C2 } & 116.1(3) \\ \text { N1-C6-H6A } & 109.5 \\ \text { N1-C6-H6B } & 109.5 \\ \text { N1-C6-H6C } & 109.5 \\ \text { H6A-C6-H6B } & 109.5 \\ \text { H6A-C6-H6C } & 109.5 \\ \text { H6B-C6-H6C } & 109.5 \\ \text { C7-N2-C9 } & 120.4(3) \\ \text { C7-N2-C8 } & 122.3(3) \\ \text { C9-N2-C8 } & 117.2(3) \\ \text { O1-C7-N2 } & 125.8(3) \\ \text { O1-C7-H7 } & 117.1 \\ \text { N2-C7-H7 } & 117.1 \\ \text { N2-C9-H9A } & 109.5 \\ \text { N2-C9-H9B } & 109.5 \\ \text { N2-C9-H9C } & 109.5 \\ \text { H9A-C9-H9B } & 109.5 \\ \text { H9A-C9-H9C } & 109.5 \\ \text { H9B-C9-H9C } & 109.5 \\ \text { N2-C8-H8A } & 109.5 \\ \text { N2-C8-H8B } & 109.5 \\ \text { N2-C8-H8C } & 109.5 \\ \text { H8A-C8-H8B } & 109.5 \\ \text { H8A-C8-H8C } & 109.5 \\ \text { H8B-C8-H8C } & 109.5 \\ \text { C10-N3-C12 } & 119.7(3) \\ \text { C10-N3-C11 } & 122.2(3) \\ \text { C11-N3-C12 } & 118.1(3) \\ \text { O2-C10-N3 } & 125.6(3) \\ \text { O2-C10-H10 } & 117.2 \\ \text { N3-C10-H10 } & 17.2 \\ \text { N3-C12-H12A } & \\ \text { N3-C12-H12B } & \\ \text { N3-C12-H12C } & \\ \text { H12A-C12-H12B } & \text { C12-H12C } \\ & \end{array}$




\begin{tabular}{|c|c|c|c|}
\hline $\mathrm{Cl} 1-\mathrm{Mo} 2-\mathrm{Cl} 4$ & $90.03(2)$ & $\mathrm{H} 12 \mathrm{~B}-\mathrm{C} 12-\mathrm{H} 12 \mathrm{C}$ & 109.5 \\
\hline Mo1 ${ }^{\mathrm{i}}-\mathrm{Mo} 3-\mathrm{Mo1}$ & $90.008(11)$ & $\mathrm{N} 3-\mathrm{C} 11-\mathrm{H} 11 \mathrm{~A}$ & 109.5 \\
\hline Mo1 - Mo3-Mo2 & $60.064(8)$ & $\mathrm{N} 3-\mathrm{C} 11-\mathrm{H} 11 \mathrm{~B}$ & 109.5 \\
\hline Mo1-Mo3-Mo2 & $59.788(11)$ & $\mathrm{N} 3-\mathrm{C} 11-\mathrm{H} 11 \mathrm{C}$ & 109.5 \\
\hline $\mathrm{Mo} 2 \mathrm{i}-\mathrm{Mo} 3-\mathrm{Mo1}$ & $60.140(9)$ & $\mathrm{H} 11 \mathrm{~A}-\mathrm{C} 11-\mathrm{H} 11 \mathrm{~B}$ & 109.5 \\
\hline $\mathrm{Mo} 2^{\mathrm{i}}-\mathrm{Mo} 3-\mathrm{Mo}^{\mathrm{i}}$ & $59.936(10)$ & $\mathrm{H} 11 \mathrm{~A}-\mathrm{C} 11-\mathrm{H} 11 \mathrm{C}$ & 109.5 \\
\hline $\mathrm{Mo} 2 \mathrm{i}-\mathrm{Mo} 3-\mathrm{Mo} 2$ & $89.930(10)$ & $\mathrm{H} 11 \mathrm{~B}-\mathrm{C} 11-\mathrm{H} 11 \mathrm{C}$ & 109.5 \\
\hline $\mathrm{Cl} 4-\mathrm{Mo} 3-\mathrm{Mo1}^{\mathrm{i}}$ & $118.345(18)$ & $\mathrm{C} 3-\mathrm{C} 2-\mathrm{H} 2$ & 119.6 \\
\hline Cl4-Mo3-Mo1 & $58.082(15)$ & $\mathrm{C} 1-\mathrm{C} 2-\mathrm{C} 3$ & $120.8(3)$ \\
\hline $\mathrm{Cl} 1-\mathrm{Mo} 3-\mathrm{Mo} 2$ & $58.296(16)$ & $\mathrm{C} 1-\mathrm{C} 2-\mathrm{H} 2$ & 119.6 \\
\hline $\mathrm{Cl} 4-\mathrm{Mo} 3-\mathrm{Mo} 2^{\mathrm{i}}$ & $118.209(17)$ & $\mathrm{N} 1-\mathrm{C} 1-\mathrm{C} 2$ & $121.2(3)$ \\
\hline $\mathrm{Cl} 4-\mathrm{Mo} 3-\mathrm{Cl} 2$ & $89.94(2)$ & $\mathrm{N} 1-\mathrm{C} 1-\mathrm{H} 1$ & 119.4 \\
\hline $\mathrm{Cl} 2-\mathrm{Mo} 3-\mathrm{Mo} 1$ & $117.894(18)$ & $\mathrm{C} 2-\mathrm{C} 1-\mathrm{H} 1$ & 119.4 \\
\hline $\mathrm{Cl} 2-\mathrm{Mo} 3-\mathrm{Mol}^{\mathrm{i}}$ & $58.152(15)$ & $\mathrm{C} 3-\mathrm{C} 4-\mathrm{H} 4$ & 119.4 \\
\hline $\mathrm{Cl} 2-\mathrm{Mo} 3-\mathrm{Mo} 2^{\mathrm{i}}$ & $118.066(17)$ & $\mathrm{C} 5-\mathrm{C} 4-\mathrm{C} 3$ & $121.1(3)$ \\
\hline $\mathrm{Cl} 2-\mathrm{Mo} 3-\mathrm{Mo} 2$ & $58.121(16)$ & $\mathrm{C} 5-\mathrm{C} 4-\mathrm{H} 4$ & 119.4 \\
\hline Cl7-Mo3-Mo1 & $135.303(19)$ & $\mathrm{N} 1-\mathrm{C} 5-\mathrm{C} 4$ & $121.2(3)$ \\
\hline $\mathrm{Cl} 7-\mathrm{Mo} 3-\mathrm{Mo}^{\mathrm{i}}$ & $134.689(19)$ & $\mathrm{N} 1-\mathrm{C} 5-\mathrm{H} 5$ & 119.4 \\
\hline $\mathrm{Cl} 1-\mathrm{Mo} 3-\mathrm{Mo} 2$ & $135.02(2)$ & $\mathrm{C} 4-\mathrm{C} 5-\mathrm{H} 5$ & 119.4 \\
\hline $\mathrm{C} 3^{\mathrm{ii}}-\mathrm{C} 3-\mathrm{C} 2-\mathrm{C} 1$ & $179.5(3)$ & $\mathrm{C} 8-\mathrm{N} 2-\mathrm{C} 7-\mathrm{O} 1$ & $176.1(5)$ \\
\hline $\mathrm{C} 3^{\mathrm{ii}}-\mathrm{C} 3-\mathrm{C} 4-\mathrm{C} 5$ & $-179.8(3)$ & $\mathrm{C} 12-\mathrm{N} 3-\mathrm{C} 10-\mathrm{O} 2$ & $-1.1(5)$ \\
\hline $\mathrm{C} 3-\mathrm{C} 2-\mathrm{C} 1-\mathrm{N} 1$ & $-0.3(5)$ & $\mathrm{C} 11-\mathrm{N} 3-\mathrm{C} 10-\mathrm{O} 2$ & $179.6(3)$ \\
\hline $\mathrm{C} 3-\mathrm{C} 4-\mathrm{C} 5-\mathrm{N} 1$ & $0.8(5)$ & $\mathrm{C} 2-\mathrm{C} 3-\mathrm{C} 4-\mathrm{C} 5$ & $-0.3(5)$ \\
\hline $\mathrm{C} 6-\mathrm{N} 1-\mathrm{C} 1-\mathrm{C} 2$ & $179.3(3)$ & $\mathrm{C} 1-\mathrm{N} 1-\mathrm{C} 5-\mathrm{C} 4$ & $-1.0(5)$ \\
\hline $\mathrm{C} 6-\mathrm{N} 1-\mathrm{C} 5-\mathrm{C} 4$ & $-179.5(3)$ & $\mathrm{C} 4-\mathrm{C} 3-\mathrm{C} 2-\mathrm{C} 1$ & $0.0(4)$ \\
\hline $\mathrm{C} 9-\mathrm{N} 2-\mathrm{C} 7-\mathrm{O} 1$ & $-0.6(7)$ & $\mathrm{C} 5-\mathrm{N} 1-\mathrm{C} 1-\mathrm{C} 2$ & $0.8(5)$ \\
\hline
\end{tabular}

Symmetry codes: (i) $-x+1,-y+1,-z+1$; (ii) $-x,-y+2,-z+1$.

Hydrogen-bond geometry $\left(\AA,{ }^{\circ}\right)$

\begin{tabular}{lllll}
\hline$D-\mathrm{H} \cdots A$ & $D-\mathrm{H}$ & $\mathrm{H} \cdots A$ & $D \cdots A$ & $D-\mathrm{H} \cdots A$ \\
\hline $\mathrm{C} 5-\mathrm{H} 5 \cdots \mathrm{O} 1^{\mathrm{iii}}$ & 0.95 & 2.23 & $3.063(4)$ & 145 \\
$\mathrm{C} 6-\mathrm{H} 6 C \cdots \mathrm{O} 2^{\mathrm{ii}}$ & 0.98 & 2.31 & $3.088(4)$ & 136 \\
\hline
\end{tabular}

Symmetry codes: (ii) $-x,-y+2,-z+1$; (iii) $x, y+1, z$. 